\title{
Placental endocrine function shapes cerebellar development and social behavior
}

\author{
Claire-Marie Vacher ${ }^{1 凶}$, Helene Lacailleㄹ, Jiaqi J. O'Reilly', Jacquelyn Salzbank1, Dana Bakalar², \\ Sonia Sebaoui ${ }^{3}$, Philippe Liere ${ }^{4}$, Cheryl Clarkson-Paredes ${ }^{5}$, Toru Sasaki ${ }^{3}$, Aaron Sathyanesan ${ }^{3}$, \\ Panagiotis Kratimenos ${ }^{3,10}$, Jacob Ellegood ${ }^{7}$, Jason P. Lerch ${ }^{7,8}$, Yuka Imamura9 , Anastas Popratiloff ${ }^{5,6}$, \\ Kazue Hashimoto-Torii $\mathbb{1}^{3,10}$, Vittorio Gallo ${ }^{3,10}$, Michael Schumacher ${ }^{4}$ and Anna A. Penn (1) $1 \times$
}

Compromised placental function or premature loss has been linked to diverse neurodevelopmental disorders. Here we show that placenta allopregnanolone (ALLO), a progesterone-derived GABA-A receptor $\left(G A B A_{A} R\right.$ ) modulator, reduction alters neurodevelopment in a sex-linked manner. A new conditional mouse model, in which the gene encoding ALLO's synthetic enzyme (akr1c14) is specifically deleted in trophoblasts, directly demonstrated that placental ALLO insufficiency led to cerebellar white matter abnormalities that correlated with autistic-like behavior only in male offspring. A single injection of ALLO or muscimol, a $G_{A B A} R$ agonist, during late gestation abolished these alterations. Comparison of male and female human preterm infant cerebellum also showed sex-linked myelination marker alteration, suggesting similarities between mouse placental ALLO insufficiency and human preterm brain development. This study reveals a new role for a placental hormone in shaping brain regions and behaviors in a sex-linked manner. Placental hormone replacement might offer novel therapeutic opportunities to prevent later neurobehavioral disorders.

$\mathrm{N}$ europlacentology is a term coined to describe an emerging research area that aims to understand the influence of placental function on the developing brain. Placental dysfunction or pathology has been associated with abnormal neurodevelopment ${ }^{1}$, but the causal mechanisms remain largely unknown. Much research connecting placental compromise to fetal brain injury has focused on gas exchange or nutritional programming, neglecting the placenta's essential neuroendocrine role. Many events, including infection, malnutrition and genetic abnormalities, can disrupt placental function or, as in preterm birth, can abruptly change the hormonal environment of the developing brain. Such changes might alter early brain development or increase the susceptibility of the immature brain to damage.

ALLO, a major GABAergic neurosteroid, might be one key placental hormone in shaping fetal brain. ALLO is synthesized from progesterone and is a positive allosteric modulator of $\mathrm{GABA}_{\mathrm{A}} \mathrm{Rs}$ that binds to specific receptor sites and prolongs the opening of the GABA-gated Cl- channel $^{2}$. In the adult brain, ALLO enhances GABAergic inhibition, producing sedative, anxiolytic, anesthetic and anticonvulsant effects ${ }^{3}$. A large body of evidence suggests that ALLO, through $\mathrm{GABA}_{\mathrm{A}} \mathrm{R}$ signaling, is also a potent regulator of many neurodevelopmental processes, including neurogenesis, neuritogenesis, cell survival, synapse stabilization and myelination ${ }^{4}$. In fetal sheep and guinea pigs, brain ALLO is increased in response to immune challenge, hypoxia and other acute prenatal stressors. Blocking this increase pharmacologically with finasteride, a $5 \alpha$-reductase inhibitor that also blocks ALLO production, leads to increased apoptosis, excitotoxicity and impaired myelination, particularly in males 5 . These observations support an endogenous neuroprotective role for ALLO and a potential therapeutic efficacy of ALLO analogs as central nervous system drugs but do not directly connect specific placental endocrine functions to changes in neurodevelopment.

Our novel mouse model of specific placental ALLO reduction allowed direct investigation of the neurodevelopmental effect of altered placental endocrine function at a molecular, cellular and functional level. A combination of microscopy, gene and protein expression analysis, neurobehavioral assessments and pharmacological rescue in this model shows that placenta ALLO insufficiency leads to cerebellar myelination abnormalities that correlate with autistic-like behaviors in a sex-linked manner. Understanding how specific placental hormones shape normal brain development and how placental loss or dysfunction contributes to the neurological impairments in those born extremely preterm or after compromised pregnancies lays the groundwork for developing hormone replacement strategies to maintain the normal developmental milieu and protect the brain from further injury.

\section{Results}

Akr1c14 deletion in Cyp19+ cells impairs placental ALLO production. During fetal development, akr1c14, the gene encoding the ALLO-synthesizing enzyme $3 \alpha$-hydroxysteroid dehydrogenase $(3 \alpha-H S D)$, is primarily expressed in the placenta, with up to 60 times higher expression than in mouse brain, and peaks in the

'Department of Pediatrics, Columbia University, New York-Presbyterian Morgan Stanley Children's Hospital, New York, NY, USA. ${ }^{2}$ National Institutes of Health, Bethesda, MD, USA. ${ }^{3}$ Center for Neuroscience Research, Children's National Health System, Washington, DC, USA. ${ }^{4}$ U1195 INSERM, Paris-Saclay University, Le Kremlin-Bicêtre Cedex, France. ${ }^{5}$ The George Washington University, Nanofabrication and Imaging Center, Washington, DC, USA. ${ }^{6}$ The George Washington University, SMHS, Anatomy \& Cell Biology, Washington, DC, USA. ${ }^{7}$ Mouse Imaging Centre (MICe), Hospital for Sick Children, Toronto, ON, Canada. ${ }^{8}$ Wellcome Centre for Integrative Neuroimaging (WIN), Nuffield Department of Clinical Neurosciences, University of Oxford, John Radcliffe Hospital, Oxford, UK. 'Department of Biochemistry and Molecular Biology, Pennsylvania State University College of Medicine, Pittsburgh, PA, USA. ${ }^{10}$ The George Washington University School of Medicine and Health Sciences, Pediatrics, Washington, DC, USA.

凶e-mail: cv2494@cumc.columbia.edu; aap2216@cumc.columbia.edu 
a

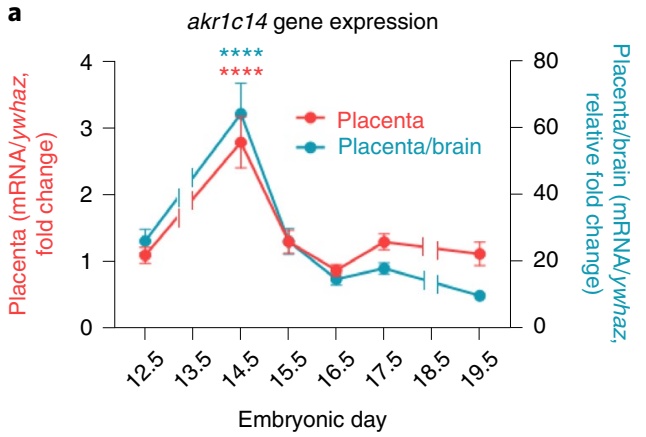

b

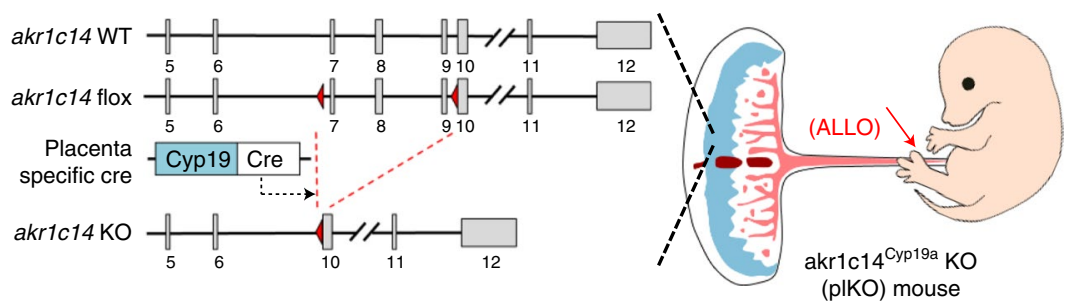

c

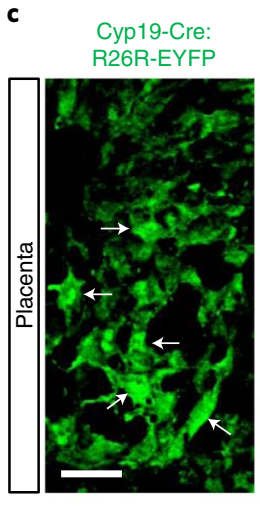

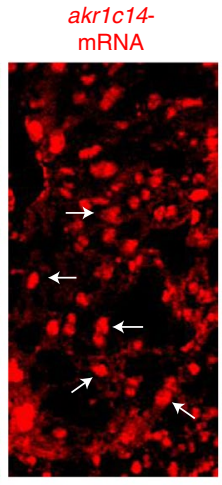
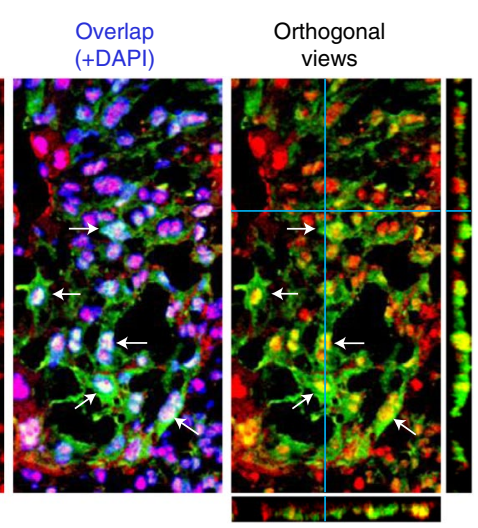

d
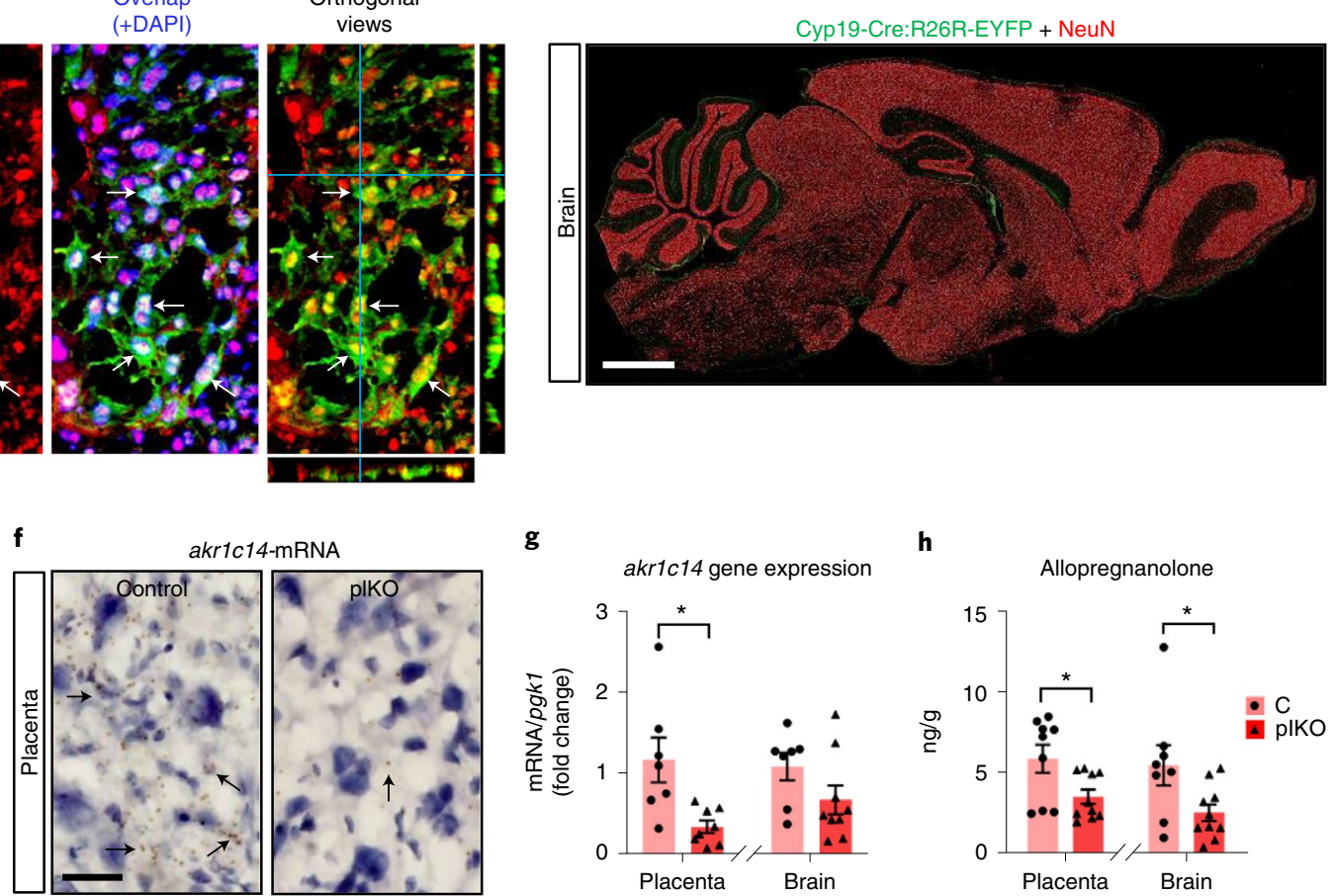

g

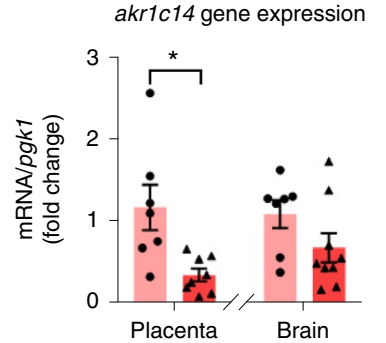

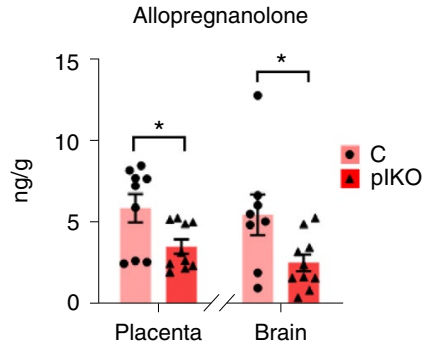

Fig. 1 | Conditional deletion of akr1c14 in Cyp19a-expressing trophoblasts results in reduced ALLO levels in the fetal brain. a, qRT-PCR for akr1c14 in wild-type (WT) mice. Data presented as mean fold changes \pm s.e.m. E12.5: $n=12 \mathrm{C}$ and 12 plKO; E14.5: $n=12 \mathrm{C}$ and 12 plKO; E15.5: $n=10 \mathrm{C}$ and 8 pIKO; E16.5: $n=7 \mathrm{C}$ and 7 plKO; E17.5: $n=12 \mathrm{C}$ and 12 pIKO; E19.5: $n=12 \mathrm{C}$ and 11 plKO. One-way ANOVA with Dunnett's multiple comparisons ( $P<0.0001$ compared to E12.5 value). There was no significant difference between males and females. ywhaz, tyrosine 3-monooxygenase/tryptophan 5-monooxygenase activation protein zeta. b, akr1c14 genetic locus before and after recombination. Exons 7-9 are conditionally targeted. Cyp19a promoter drives expression of Cre in the placenta only. LoxP sites are indicated by red triangles and exons 5-12 by gray boxes. c, In situ hybridization for akr1c14 in placenta from Cyp19a-Cre:R26R-EYFP mice at E17.5. Arrows show co-localizations. Scale bar, $50 \mu m$. d, Sagittal section of a Cyp19a-Cre:R26R-EYFP mouse brain immunostained against YFP (green) and NeuN (red). No Cyp19a promotor activity was evidenced in the brain at P30. Scale bar, 1 mm. e, A 164-bp-long PCR product ascertains the presence of the recombined LoxP site in the placenta of plKO mice. $\mathbf{f}$, In situ hybridization for akr1c14 in the placenta at E17.5. akr1c14-mRNA levels are drastically decreased in the spongiotrophoblasts (arrows) of pIKO compared to C mice. Scale bar, 30 um. $\mathbf{g}$, qRTPCR for akr1c14 normalized to pgk1 at E17.5. Data are presented as mean \pm s.e.m. Two-tailed unpaired $t$-test with Welch's correction ( ${ }^{\star} P<0.05$ ). Placenta: $n=7 \mathrm{C}$ and 8 pIKO ( $P=0.0247)$; Brain: $n=8 \mathrm{C}$ and 9 pIKO ( $P=0.122)$. Pgk1, phosphoglycerate kinase 1. h, Mass spectrometry ALLO assays at E17.5. Data are presented as mean \pm s.e.m. Two-tailed unpaired $t$-test with Welch's correction $\left({ }^{\star} P<0.05\right)$. Placenta: $n=9 \mathrm{C}$ and 10 plKO $(P=0.0315)$; Brain: $n=8 \mathrm{C}$ and 10 pIKO $(P=0.0329)$.

second half of gestation (Fig. 1a). To assess the neurodevelopmental role of placental ALLO, we generated akr1c14-floxed mice and crossed them with placenta-specific Cyp19a-Cre mice ${ }^{6}$ (Fig. 1b). Recombinase activity was confirmed specifically in placenta (Fig. 1c) but not in brain (Fig. 1d), as evidenced by crossing Cyp19-Cre with R26R-EYFP reporter mice. Akr1c14-mRNA co-localized with Cyp19a-Cre:R26R-EYFP-positive cells (arrows, Fig. 1c). Polymerase chain reaction (PCR) confirmed recombination of LoxP sites in placenta, but not brain, of akr1c14 ${ }^{\text {Cyp19a }}$ knockout $(\mathrm{KO})$ mice (plKO for placental conditional $\mathrm{KO}$ ) and no recombination in placenta or brain of control (C) animals (Fig. 1e). In the plKO mice, akr1c14 transcript was significantly reduced in the placenta but not brain (Fig. 1f,g). Direct steroid measurements revealed that ALLO was significantly reduced in the placenta and fetal brain in late gestation (Fig. 1h and Supplementary Table 1). The highest gestational conversion rate of ALLO (based on progesterone (PROG)/ALLO ratio) in $\mathrm{C}$ mice coincided with the akrlc14 gene expression peak at embryonic day 14.5 (E14.5) (Supplementary Table 1 and Fig. 1a). Thus, akr1c14 placental gene removal results in greatest placental ALLO insufficiency in the second half of mouse gestation (E14.5). 
The positive placenta-brain correlation of ALLO levels is consistent with placental provision of ALLO to the fetal brain (Extended Data Fig. 1a). Placental ALLO levels were not altered by sex (Extended Data Fig. 1a). ALLO precursors and other neuroactive steroids in the synthesis pathway were not altered (Supplementary Table 1 and Extended Data Fig. 1b-f), with the exception of a transient E14.5 reduction of placental allotetrahydrodeoxycorticosterone (ALLOTHDOC), which is also converted by placental $3 \alpha-\mathrm{HSD}$ (Supplementary Table 1). Placental ALLOTHDOC concentrations are negligible when compared to ALLO levels, suggesting that major effects in the plKO are primarily due to placental ALLO insufficiency (Supplementary Table 1). Additionally, the equivalent and specific decrease in both brain and placenta of plKO ALLO levels supports the specificity of this model (Fig. 1h and Extended Data Fig. 1b-f).

Placental ALLO loss alters postnatal cerebellar myelination. Unbiased RNA sequencing (RNA-seq) analysis of cerebral cortex, hippocampus and cerebellum of $\mathrm{C}$ and plKO mice at postnatal day 30 (P30) in both sexes was performed to define the most prominent postnatal molecular alterations associated with placental ALLO insufficiency. Threshold criteria used to identify significant differentially expressed genes (DEGs) were validated by RT-PCR and western blots (Extended Data Fig. 2). Male cerebellum was the brain structure affected most by placental ALLO deficiency as measured by the number of DEGs, which was 3-5 times higher than in any other brain region (Extended Data Fig. 3a,b and Supplementary Table 2, sheets 1 and 2). Cerebellar DEGs were equally distributed in up-regulated and down-regulated gene categories (Extended Data Fig. 3a-d). Ingenuity Pathway Analysis (IPA, Qiagen) identified white matter (WM)-associated pathways in the top categories of altered genes in both sexes (Extended Data Fig. 3e,f). Comparison of cerebellar DEGs to previous published oligodendrocyte (OL) and myelin transcriptomes ${ }^{7,8}$ revealed an overlap of 204 genes and 68 genes in males and females, respectively (Extended Data Fig. 3g). Striking sexual dimorphism was seen: male and female DEGs were qualitatively different, and most OL-/myelin-related DEGs were up-regulated in plKO males but down-regulated in plKO females (Extended Data Fig. 3h,i).

Investigation of the cerebellar myelin proteins at P30 revealed major sex-linked changes: in plKO males, myelin basic protein (MBP), myelin-associated glycoprotein (MAG) and myelin oligodendrocyte glycoprotein (MOG) levels were all significantly increased (Fig. 2a,b), whereas, in plKO females, MBP cerebellar content was reduced (Fig. 2c,d) - changes confirmed by immunohistochemistry (Fig. 2e-h) and RT-PCR (Extended Data Fig. 2h).
Similar sex-dependent MBP changes were previously described in the cerebellum of guinea pigs delivered preterm ${ }^{9}$. In addition, a migration downshift of MAG on western blots was observed in plKO males but not females (Fig. 2a,c), suggesting increased myelin maturation in males ${ }^{10}$. Further structural characterization of the cerebellar WM within lobule VI-VII by scanning electron microscopy (Fig. 2i) revealed thicker myelin (g-ratio measurements; Fig. 2j,k,l,o,s) and fewer unmyelinated axons in male plKOs (Fig. 2p). This increased myelin thickness was not associated with significant changes in axonal inner calibers (Fig. 2t). In contrast, cerebellar axons in plKO females had significantly thinner myelin sheath, and more axons were unmyelinated (Fig. $2 \mathrm{~m}, \mathrm{n}, \mathrm{q}, \mathrm{r}, \mathrm{u}$ ), although axonal inner diameters were unchanged (Fig. 2v) compared to C littermates.

A global comparison of regional, sex-linked and genotype-specific brain anatomy was performed by magnetic resonance imaging (MRI) at P30. Most notably, the mean fractional anisotropy (FA) of the cerebellar fiber tracts, measured by diffusion tensor imaging (DTI), was significantly increased in the plKO males, but not females, at P30 (Extended Data Fig. 4a; region effect: $F_{25,1014}=57.36$, $P<0.0001$; genotype effect: $F_{1,1014}=16.99, P<0.0001$; sex $\times$ genotype interaction: $F_{1,1014}=17.92, P<0.0001$; three-way analysis of variance (ANOVA)). In contrast, mean FAs in other WM tracts outside of the cerebellum were not significantly different in plKO mice compared to their $\mathrm{C}$ littermates (Extended Data Fig. 4b; region effect: $F_{8,351}=80.28, P<0.0001$; genotype effect: $F_{1,351}=0.1618, P=0.6877$; sex $\times$ genotype interaction: $F_{1,351}=2.589, P=0.1021$; three-way ANOVA). The male-specific increase in cerebellar FA is consistent with the enhanced degree of myelination ${ }^{11}$. OL-/myelin-related DEGs, including $m b p$, were mostly down-regulated in both male and female cerebral cortex in plKO mice (Extended Data Fig. 5). Both the DTI results and RNA-seq transcriptome patterns suggest overall sex-linked and regionally specific myelin abnormalities after placental ALLO loss.

Postnatal OL differentiation rate diverges in plKO males and females. Cerebellar myelination begins in late mouse gestation and is primarily a postnatal process ${ }^{12-14}$, so placental endocrine disruption leading to myelination anomalies was unanticipated. The adjustment of myelin sheath thickness notably relies on the axon diameter, whose distribution was unchanged in plKO cerebellum (Fig. 2t,v) and the local density of myelin-competent OLs that are primarily produced before birth as OL progenitor cells (OPCs) ${ }^{14}$, at a time when ALLO is highest. ALLO is a potent positive allosteric modulator of $\mathrm{GABA}_{\mathrm{A}} \mathrm{R}^{2}$. $\mathrm{GABA}_{\mathrm{A}} \mathrm{R}$ signaling is associated with

Fig. 2 | Placental ALLO insufficiency results in sex-linked cerebellar WM abnormalities at P30. a,b, Western blot analysis of myelin-related proteins in the male cerebellum. Data are presented as mean fold changes \pm s.e.m. Multiple unpaired $t$-tests with Holm-Sidak multiple comparison test $\left({ }^{\star} P<0.05\right.$; $\left.{ }^{* \star *} P<0.005\right)$. MBP: $n=12 \mathrm{C}$ and 28 pIKO $(P=0.038)$; MAG: $n=6 \mathrm{C}$ and 6 pIKO $(P=0.004) ;$ MOG: $n=6 \mathrm{C}$ and 6 plKO $(P<0.0001)$. c,d, Western blot analysis of myelin-related proteins in the female cerebellum. Data are presented as mean fold changes \pm s.e.m. Multiple unpaired $t$-tests with Holm-Sidak multiple comparison test $\left({ }^{\star} P<0.05\right)$. MBP: $n=5 \mathrm{C}$ and 4 pIKO $(P=0.031) ; \mathrm{MAG}: n=5 \mathrm{C}$ and 4 pIKO $(P=0.18) ; \mathrm{MOG}: n=5 \mathrm{C}$ and 4 pIKO $(P=0.54)$. GAPDH, glyceraldehyde 3-phosphate dehydrogenase. $\mathbf{e - h}$, Immunofluorescent staining of MBP in cerebellar lobule VII in males (e and $\mathbf{f}$ ) and females (g and $\mathbf{h}$ ). Scale bar, $150 \mu \mathrm{m}$. i, Scanning electron microscopy acquisition of a whole cerebellum ultrathin section showing the different cerebellar lobules (I-X) in a control mouse ( $\times 650$ low magnification). The region of interest (inter-lobule VI-VII) for high-magnification acquisitions and g-ratio quantifications is indicated by the yellow rectangle. Scale bar, $300 \mu \mathrm{m}$. $\mathbf{j}$, Schematic representation of an axon and its myelin sheath illustrating g-ratio. $r$, axon inner diameter; R, axon outer diameter. $\mathbf{k}-\mathbf{n}$, Representative scanning electron microscopy acquisitions $(\times 20,000)$ of myelinated axons in inter-lobule VIVII WM in males ( $\mathbf{k}$ and $\mathbf{I}$ ) and females ( $\mathbf{m}$ and $\mathbf{n}$ ). Scale bar, $300 \mathrm{~nm}$. $\mathbf{0}$, Scatter plot of g-ratios of $>400$ individual axons in males; data are from one representative control and one representative pIKO mouse. Fitted lines are linear regressions. p. Percent of unmyelinated axons. Data are presented as mean \pm s.e.m. Two-tailed unpaired Student's $t$-test with Welch's correction $\left({ }^{\star} P<0.05\right) . n=3 \mathrm{C}$ and 3 plKO $(P=0.0275)$. $\mathbf{q}$, Scatter plot of $g$-ratios of $>400$ individual axons in females; data are from one representative control and one representative pIKO mouse. Fitted lines are linear regressions. $\mathbf{r}$, Percent of unmyelinated axons. Data are presented as mean \pm s.e.m. Two-tailed unpaired Student's $t$-test with Welch's correction $\left({ }^{\star} P<0.05\right) . n=3 \mathrm{C}$ and 3 pIKO $(P=0.0194)$. s, g-ratios by axon caliber category in males. Data are presented as mean \pm s.e.m. Two-way ANOVA with Sidak's multiple comparisons test $\left({ }^{\star} P<0.05\right) . n=3 C$ and 3 pIKO ( $\left.<1 \mu \mathrm{m}: P=0.0299 ;>1 \mu \mathrm{m}: P=0.0165\right)$. $\mathbf{t}$, Axon caliber distribution in males $(n=3 C$ and 3 plKO). u, g-ratios by axon caliber category in females. Data are presented as mean \pm s.e.m. Two-way ANOVA with Sidak's multiple comparisons test $\left({ }^{\star} P<0.05\right) . n=3 \mathrm{C}$ and 3 pIKO $<1 \mu \mathrm{m}$ : $P=0.0162 ;>1 \mu \mathrm{m}: P=0.1185)$. $\mathbf{v}$, Axon caliber distribution in females $(n=3 \mathrm{C}$ and $3 \mathrm{plKO})$. 
blockade of OPC proliferation ${ }^{15,16}$, so we tested the hypothesis that prenatal ALLO insufficiency enhances OLs production.

We found that the density of OL lineage cells (Olig2 ${ }^{+}$), and particularly mature OLs $\left(\mathrm{CCl}^{+}\right)$, were significantly increased in the male plKO cerebellum at P30 compared to controls (Extended Data Fig. $6 \mathrm{a}, \mathrm{b})$. Increased OPC proliferation during fetal life is suggested by the results of co-labelling with BrdU at E15.5 and Olig2 expression at P30 (Extended Data Fig. 6c). Additionally, within the OL lineage, an acceleration of the maturation from PDGFR $\alpha^{+}$OPCs to mature $\mathrm{CC}^{+}$OLs was observed in the plKO mice at P15 (Extended Data Fig. 6d-i). Interestingly, a sex-divergent progression of the OL lineage was observed at P15. At this age, whereas the density of Olig2 ${ }^{+}$cells was increased in plKO females in the same range as in plKO males (Extended Data Fig. 6d,j), the OL maturation (given by the $\mathrm{CCl}^{+} /$ Olig2 ${ }^{+}$cell number ratio) was reduced (Extended Data Fig. 6h,k). Overall, these results are consistent with the ultrastructural analysis a

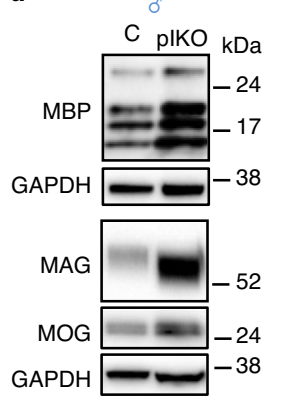

b

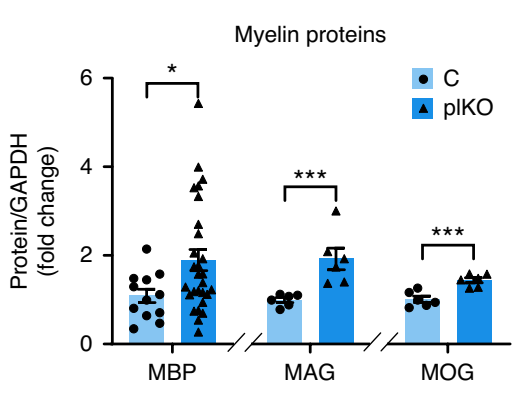

c

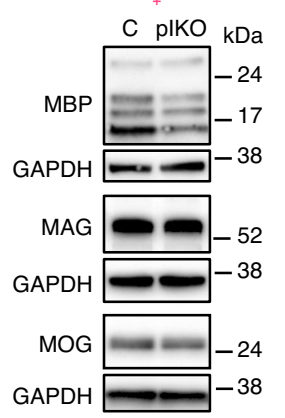

d

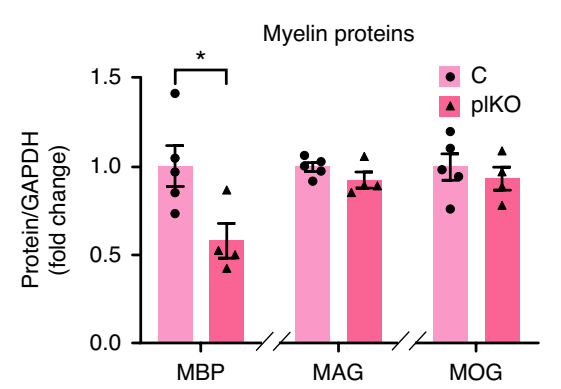

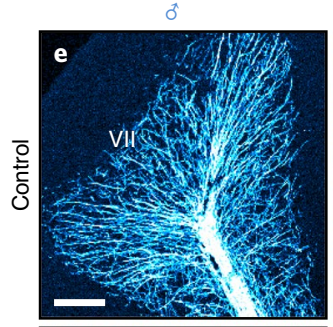
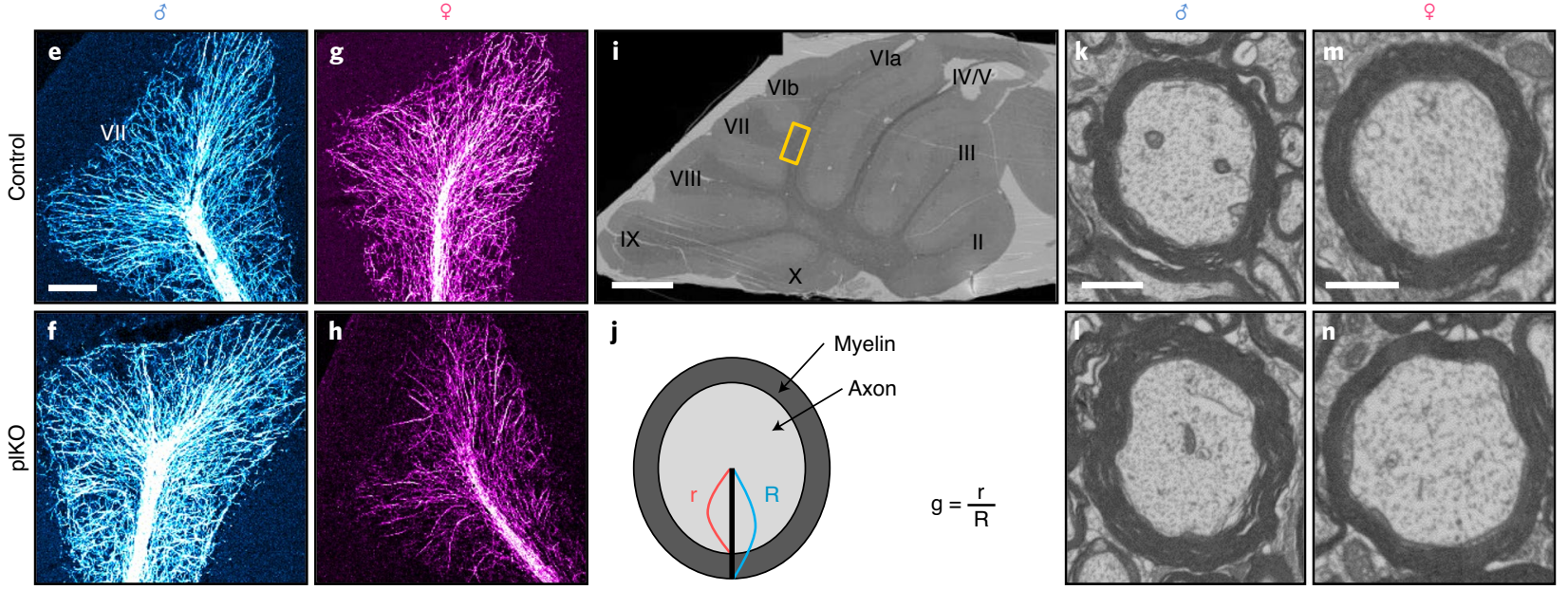

MBP

SEM
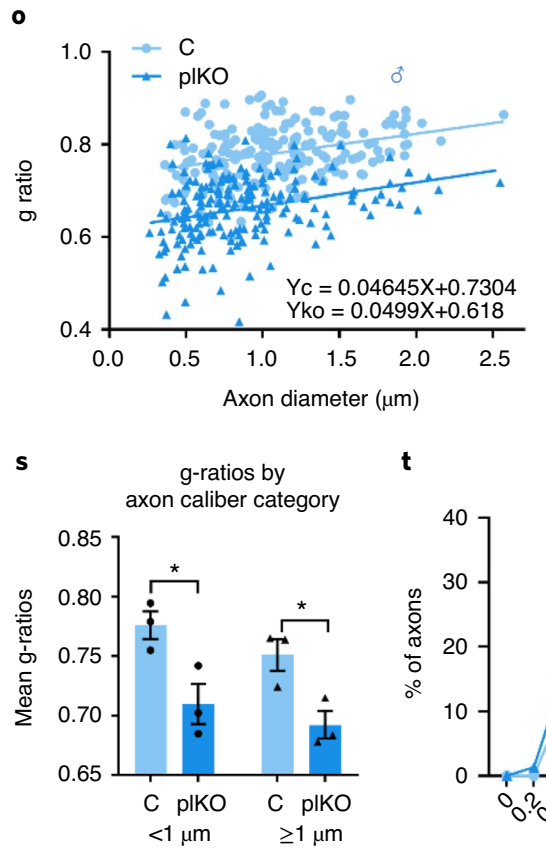

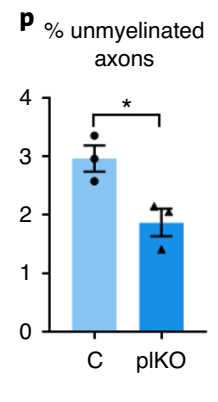

$\mathbf{t}$

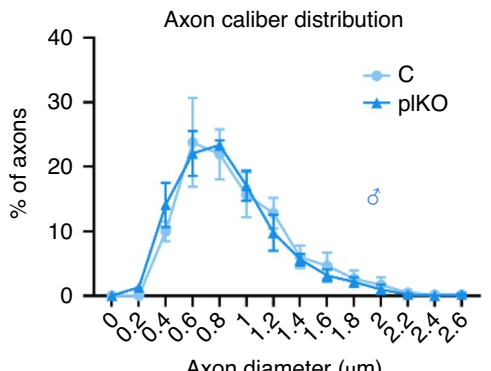

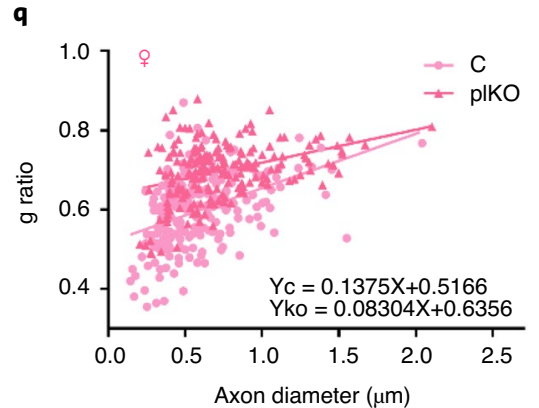

$$
\mathbf{r} \% \text { unmyelinated }
$$

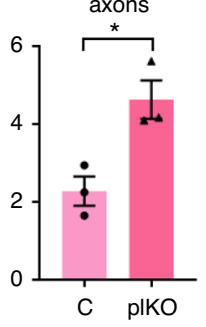

$\mathbf{u}$

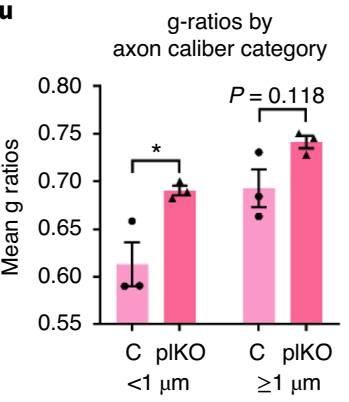

$\mathbf{v}$

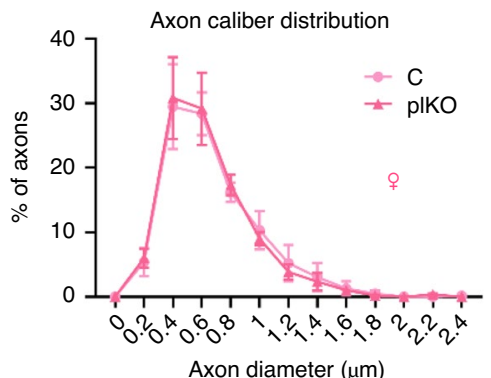




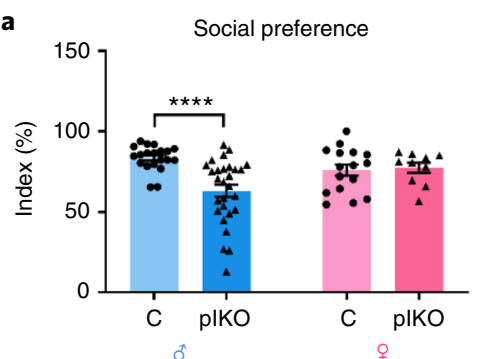

d

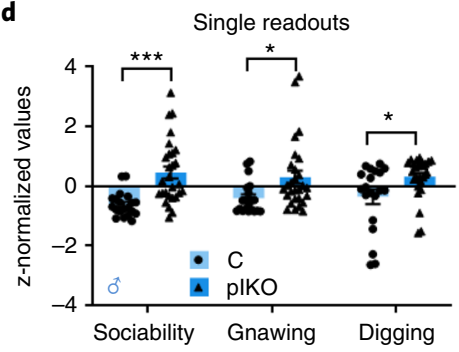

g
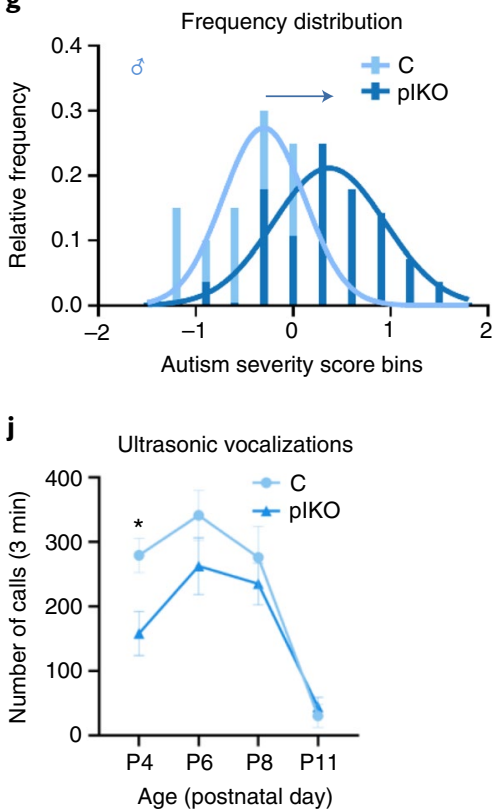

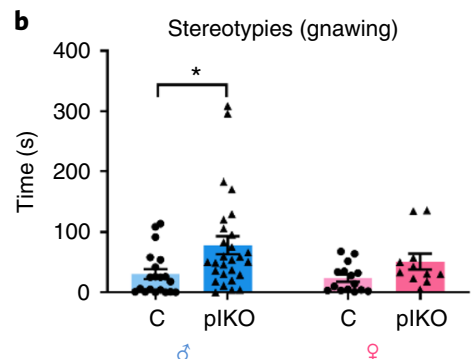

e

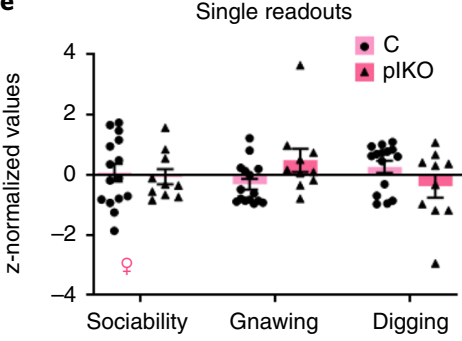

h
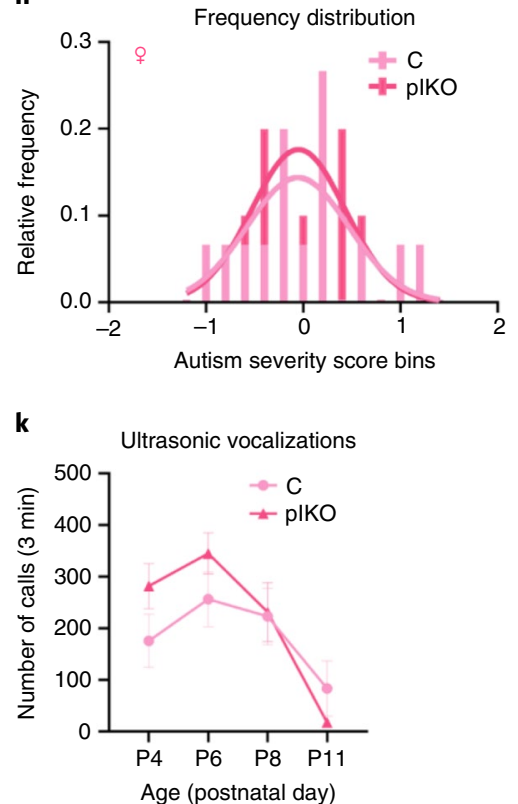

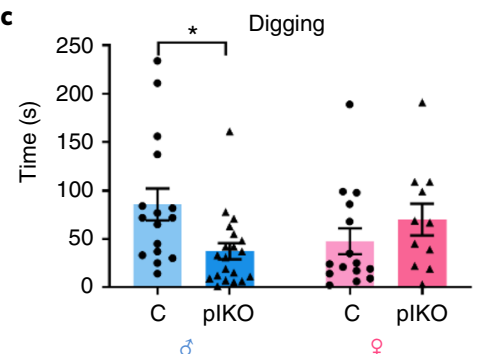

f Autism composite score

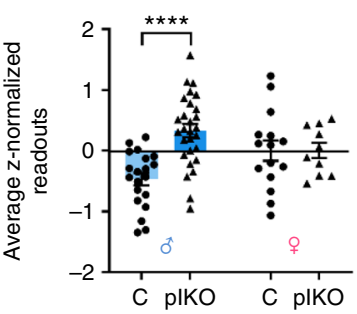

i

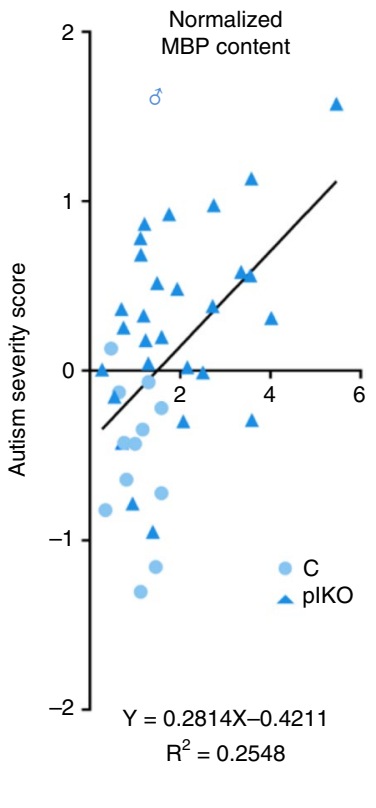

Fig. 3 | Male pIKO mice exhibit ASD-like behavior. a, Three-chamber sociability test at P30. Data are presented as mean \pm s.e.m. Two-way ANOVA with Sidak's multiple comparison test ( $\left.{ }^{\star \star \star \star} P<0.0001\right)$. Males: $n=20 \mathrm{C}$ and $28 \mathrm{plKO}(P=0.0001)$; Females: $n=16 \mathrm{C}$ and 10 plKO $(P>0.9999)$. b, Spontaneous gnawing time over $15 \mathrm{~min}$ at P30. Data are presented as mean \pm s.e.m. Two-way ANOVA with Sidak's multiple comparison test $\left({ }^{\star} P<0.01\right)$. Males: $n=20 \mathrm{C}$ and $28 \mathrm{pIKO}(P=0.0094) ;$ Females: $n=15 \mathrm{C}$ and $11 \mathrm{pIKO}(P=0.3982)$. c, Spontaneous digging time over $15 \mathrm{~min}$ at $P 30$. Data are presented as mean \pm s.e.m. Two-way ANOVA with Sidak's multiple comparison test $\left({ }^{\star} P<0.05\right)$. Males: $n=20 \mathrm{C}$ and 28 plKO $(P=0.0148)$; Females: $n=15 \mathrm{C}$ and 11 pIKO $(P=0.4781)$. d, $z$-standardized single significant behavior readouts that are integrated into the autism composite score in males at $P 30$. Data are presented as mean \pm s.e.m. Two-way ANOVA with Sidak's multiple comparisons test ( $\left.{ }^{\star} P<0.05 ;{ }^{\star \star \star} P<0.0005\right) . n=20 \mathrm{C}$ and 28 plKO. Sociability: $P=0.0002$; Gnawing: $P=0.0239$; Digging: $P=0.0301$. e, $z$-standardized single significant behavior readouts that are integrated into the autism composite score in females at P30. Data are presented as mean \pm s.e.m. Two-way ANOVA with Sidak's multiple comparisons test. $n=15 \mathrm{C}$ and 10 pIKO. Sociability: $P=0.9802$; Gnawing: $P=0.1419$; Digging: $P=0.2979$. f, ASD composite severity score at $P 30$. Data are presented as mean \pm s.e.m. Two-way ANOVA with Sidak's multiple comparison test ( $\left.{ }^{\star \star \star \star} P<0.0001\right)$. Males: $n=20 \mathrm{C}$ and 28 plKO $(P<0.0001)$; Females: $n=16 \mathrm{C}$ and 10 plKO $(P>0.9997)$. g,h, Relative frequency distribution of autism composite score bins at P30. Males: $n=20 \mathrm{C}$ and 28 pIKO; Females: $n=16 \mathrm{C}$ and 10 pIKO. i, Positive correlation between cerebellum MBP levels determined by western blot (normalized with GAPDH) and autism severity score in males at P30 ( $n=12 \mathrm{C}$ and 28 pIKO). Linear regression. Deviation from zero: $P=0.0009$. j,k, USVs at P4, P6, P8 and P11. Individual pups, males (j) and females (k), were separated from the dam and littermates, and their calls were recorded for $3 \mathrm{~min}$. Data are presented as mean \pm s.e.m. Two-way repeated measures ANOVA followed by Sidak's multiple comparisons test $\left({ }^{\star} P<0.05\right)$. Males: $n=13 \mathrm{C}$ and 15 plKO (P4: $\left.P=0.0378 ; \mathrm{P} 6: P=0.5714 ; P 8: P=0.9299 ; P 11: P=0.9609\right) ;$ Females: $n=7 \mathrm{C}$ and 7 plKO (P4: $P=0.4587 ; P 6: P=0.6051 ; P 8: P>0.9999 ; P 11: P=0.7135)$. GAPDH, glyceraldehyde 3-phosphate dehydrogenase.

of myelin and indicate short-term, sex-independent (embryonic OPC proliferation) and long-term, sex-dependent (OL maturation) modifications of cerebellar OL lineage progression and OL maturation in mice lacking placental ALLO. No volumetric change in the different cerebellar layers (Extended Data Fig. 7a-i) nor Purkinje cell linear density (Extended Data Fig. 7j) were observed in the plKO mice. 


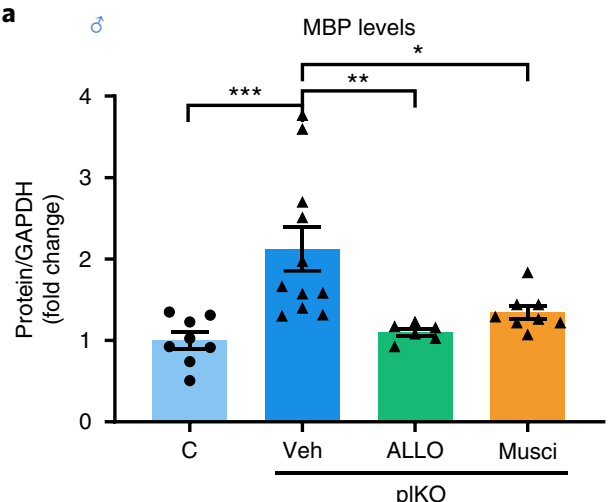

b
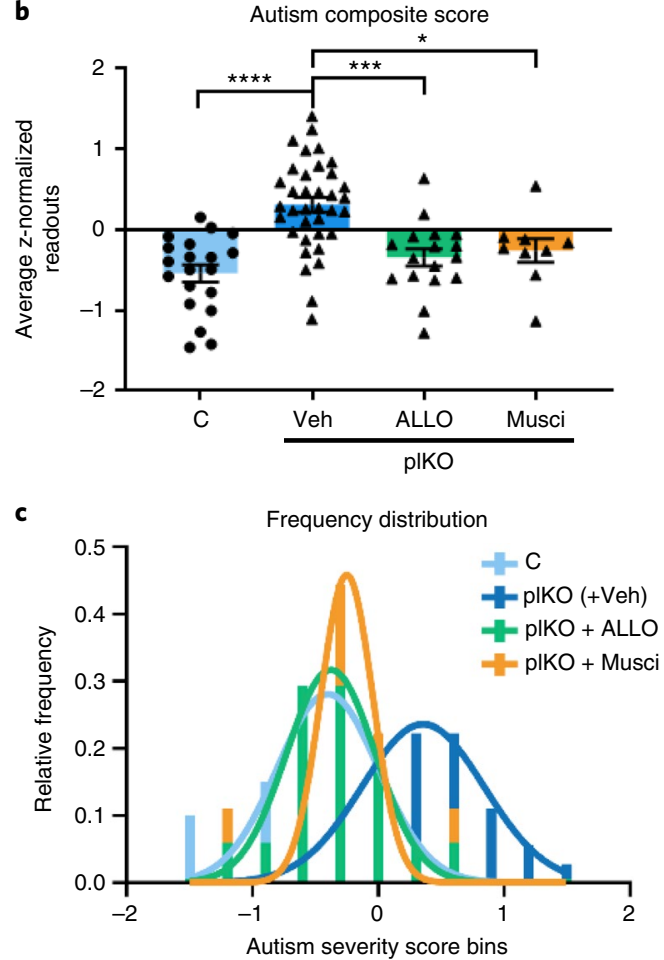

Fig. 4 | ALLO or muscimol administration during late gestation rescues MBP expression and abnormal behaviors in pIKO males at P30. a, Western blot determination of MBP contents in the cerebellum at P30. Normalized data to GAPDH contents are presented as mean fold changes \pm s.e.m. Dams received an intraperitoneal injection of ALLO $\left(10 \mathrm{mg} \mathrm{kg}^{-1}\right)$ or muscimol (Musci; $1 \mathrm{mg} \mathrm{kg}^{-1}$ ) at E15.5. One-way ANOVA with Tukey's multiple comparisons test $\left({ }^{\star} P<0.05 ;{ }^{\star \star} P<0.01 ;{ }^{\star \star \star} P<0.005\right) . n=8 \mathrm{C}$, 11 pIKO+Veh, 6 pIKO+ALLO and 8 pIKO+muscimol. Comparisons: $C$ versus pIKO+Veh: $P=0.009 ; C$ versus pIKO+ALLO: $P=0.988 ; C$ versus plKO+muscimol: $P=0.607$; IKO+Veh versus pIKO+ALLO: $P=0.0058$; pIKO+Veh versus pIKO+muscimol: $P=0.027$; pIKO+ALLO versus pIKO+muscimol: $P=0.8436 . \mathbf{b}, \mathbf{c}, A S D$ composite score and frequency distribution of autism severity score bins at P30. pIKO and pIKO+Veh mice were combined because there was no significant difference between both groups (named pIKO $(+V e h)$ ). One-way ANOVA with Tukey's multiple comparisons test ${ }^{\star \star \star} P<0.005$; $\left.{ }^{\star \star \star \star} P<0.0005\right) . n=20 \mathrm{C}, 3$ $6 \mathrm{plKO}(+\mathrm{Veh}), 17 \mathrm{pIKO}+\mathrm{ALLO}$ and 9 plKO+muscimol. Comparisons: $\mathrm{C}$ versus pIKO(+Veh): $P<0.0001 ; C$ versus pIKO+ALLO: $P=0.6226 ; C$ versus pIKO+muscimol: $P=0.4933 ; \mathrm{pIKO}(+V e h$ ) versus pIKO+ALLO: $P=0.0002 ; \mathrm{plKO}$ (+Veh) versus pIKO+muscimol: $P=0.0187$; $\mathrm{pIKO}+\mathrm{ALLO}$ versus plKO+muscimol: $P=0.9765$. GAPDH, glyceraldehyde 3-phosphate dehydrogenase.
Placental ALLO insufficiency leads to autism spectrum disorder-like behaviors in males. Cerebellar circuits provide precise spatiotemporal control of sensorimotor behavior, and cerebellar contribution to cognitive functions has more recently been recognized. Thus, developmental disruption of cerebellar circuits, either through neuronal or glial alterations, might modify motor and cognitive performance. Cerebellar-dependent tests were first selected to assess locomotor and motor learning behaviors. Behavior testing on the Erasmus ladder, an advanced, fully automated system for comprehensive real-time monitoring of cerebellar-mediated motor function, was performed at P30, the earliest validated age ${ }^{17}$, to align with the anatomical analysis. At baseline, male plKO mice exhibited a mild alteration of their walking pattern, with higher percentage of long steps (Extended Data Fig. 8a,b). The frequency of missteps, often increased in ataxia models, was unchanged (Extended Data Fig. 8g). The locomotion speed was similar in male plKO and C on the Erasmus ladder, consistent with open field testing results (Extended Data Fig. 8c,d). An associative cerebellar learning task pairing a tone with a ladder obstacle revealed no cerebellar motor learning deficit in male plKO mice (Extended Data Fig. 8e,f). Female plKOs exhibited no locomotor alterations nor learning deficits (Extended Data Fig. 8i-n). Overall, our data suggest that plKO mice do not display major locomotor, ataxia-like or associative motor learning alterations. The only abnormal locomotion feature identified in plKO mice on the Erasmus ladder was an increased frequency of long steps by males. More frequent long steps or longer stride was previously observed in genetic mouse models with impeded intrinsic or synaptic Purkinje cell plasticity ${ }^{18,19}$. However, contrasting outcomes on the Erasmus ladder were also described in mice with related physiological impairments ${ }^{20}$. Future studies focusing on Purkinje cell physiology might elucidate the precise mechanisms underlying the subtle walking pattern abnormalities seen in plKO males. Interestingly, longer stride was also reported as an autism spectrum disorder (ASD)-associated feature in human and mouse ${ }^{19,21}$.

To further evaluate balance, motor coordination and learning, mice were tested on an accelerating rotarod. Trained male, but not female, plKO mice displayed longer latency to fall and increased terminal speed (Extended Data Fig. 9a,b). The learning rate of individual mice, given by a linear regression analysis, was also enhanced in male plKO mice (Extended Data Fig. 9c,d). Greater learning in plKO males on the accelerating rotarod might be linked to their increased cerebellar FA, as previously described ${ }^{22}$. This gain of function, although rare, has been described in ASD mouse models, in which it has been attributed to increased stereotyped motor routine $^{23}$. Although the underlying molecular mechanisms and circuits might differ between models, similar repetitive/stereotypical behavior could play a role in the enhanced rotarod learning displayed by plKO males.

Beside its role in motor learning and coordination, the cerebellum contributes to cognitive processing and emotional control. Cerebellar impairments are often associated with ASD symptoms in humans and genetic mouse model ${ }^{24,25}$. Specifically, cerebellar WM abnormalities, including transient hyperplasia or dysregulation of myelin-related genes, were reported in patients with $\mathrm{ASD}^{26,27}$. We next asked whether mice lacking placental ALLO show autistic-like behavioral features by evaluating adult sociability and motor stereotypies, as well as isolation calling response at young ages. The three-chamber social behavior test revealed significant social interaction deficits in plKO males but not females (Fig. 3a). Stereotyped motor behavior was then investigated in spontaneously behaving mice. Male, but not female, plKO mice exhibited specific motor stereotypies, particularly increased gnawing (Fig. 3b). In addition, male plKO mice spent less time digging spontaneously or during a marble burying test (Fig. 3c and Extended Data Fig. 9e). Difficulties in social interactions and motor stereotypies are two ASD hallmarks, 
a
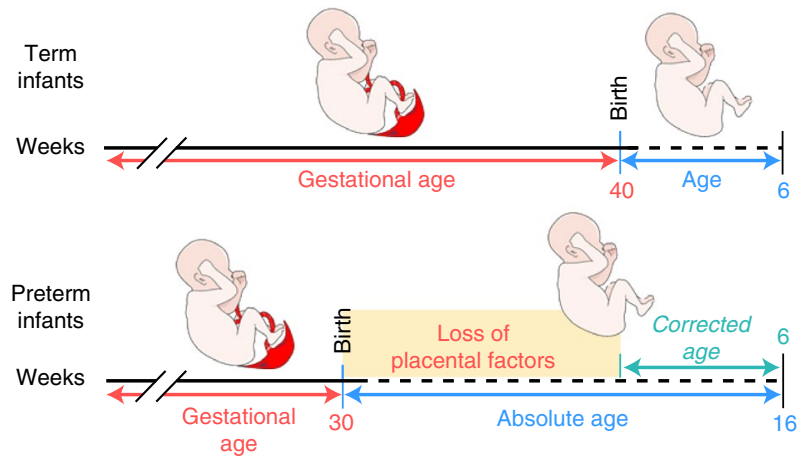

d

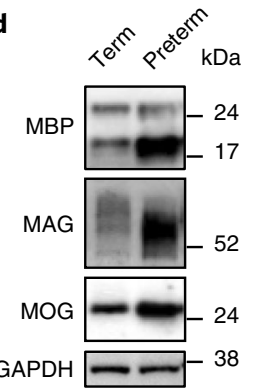

e

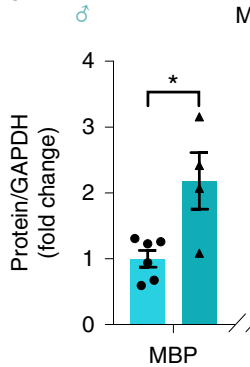

Myelin proteins

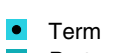

b

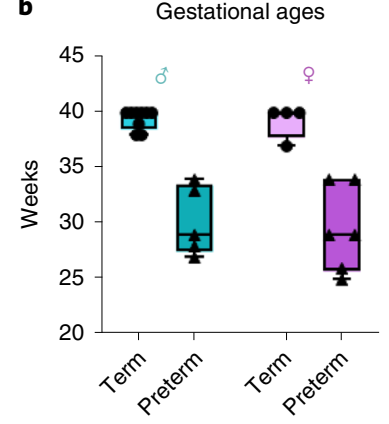

C Corrected ages at death

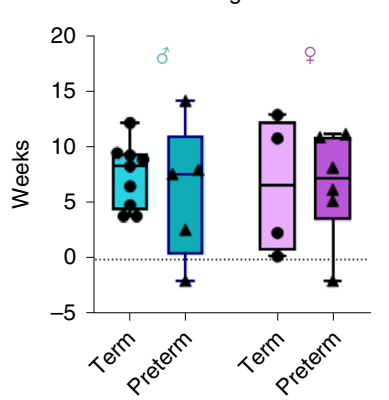

h

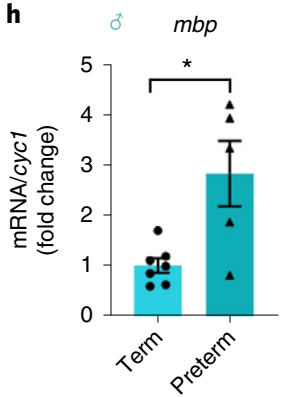

i

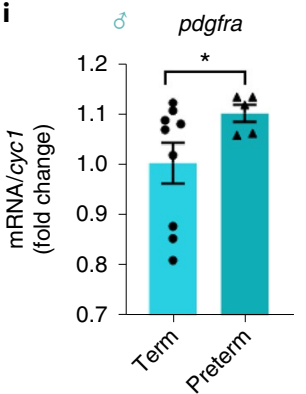

$f$

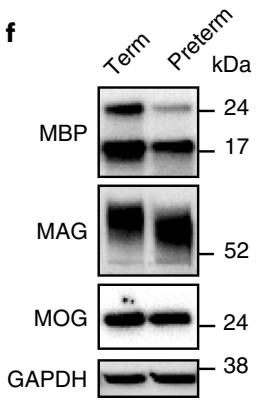

\section{g}

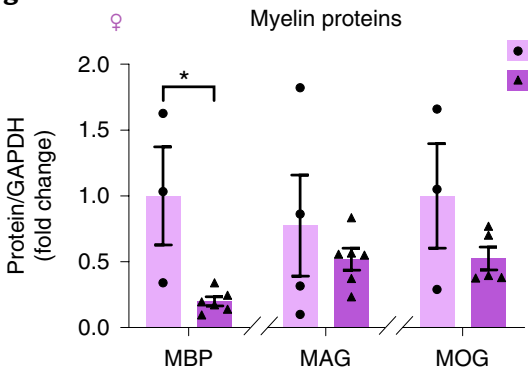

- Term

^ Preterm

Fig. 5 | Myelin proteins are dysregulated in a sex-linked manner in the cerebellar vermis of preterm infants. a, Schematic of the age terminology during the perinatal period used for the human study. b. Distribution of donors' gestational age at birth. c, Distribution of donors' corrected age at death. Graphs in $\mathbf{b}$ and $\mathbf{c}$ show box and whisker plots (including minima, maxima and median values) with single values. $\mathbf{d}$,e, Western blot analysis of myelin-related proteins in the cerebellar vermis of term and preterm male infants at an average corrected age of 6 weeks. Data are presented as mean fold changes \pm s.e.m. $(n=6 \mathrm{~T}$ and $4 \mathrm{PT})$. Multiple unpaired $t$-tests with Holm-Sidak multiple comparison test ( $\left.{ }^{\star} P<0.05\right)$. MBP: $P=0.014 ;$ MAG: $P=0.044 ;$ MOG: $P=0.024 . \mathbf{f}, \mathbf{g}$, Western blot analysis of myelin-related proteins in the cerebellar vermis of term and preterm female infants at an average corrected age of 6 weeks. Data are presented as mean fold changes \pm s.e.m. Multiple unpaired $t$-tests with Holm-Sidak multiple comparison test ( $\left.{ }^{\star} P<0.05\right)$. MBP: $n=3$ T and 6 PT ( $\left.P=0.015\right)$; MAG: $n=3$ T and 6 PT ( $\left.P=0.169\right) ;$ MOG: $n=3$ T and 5 PT $(P=0.179)$. h-j. qRT-PCR for myelin- and OL-related genes normalized to $c y c 1$ in male infants. Data are presented as mean fold changes \pm s.e.m. Two-tailed unpaired Student's $t$-test with Welch's correction ( $\left.{ }^{\star} P<0.05,{ }^{\star \star \star \star} P<0.0001\right) . m b p: ~ n=7$ T and 5 PT $(P=0.046) ; p d g f r a: ~ n=9$ T and 5 PT $(P=0.0482) ; c s p g 4: n=9$ T and 5 PT ( $\left.P<0.0001\right)$. $\mathbf{k}, \mathbf{l}$, qRT-PCR for myelin- and OL-related genes normalized to cyc1 in female infants. Data are presented as mean fold changes \pm s.e.m. Two-tailed unpaired Student's $t$-test with Welch's correction. mbp: $n=4$ T and 6 PT ( $P=0.9923)$; $p d g f r a: ~ n=4$ T and 6 PT ( $P=0.9122)$. Cspg4, chondroitin sulfate proteoglycan 4 gene; Cyc1, cytochrome C1 gene; GAPDH, glyceraldehyde 3-phosphate dehydrogenase; Pdgfra, platelet-derived growth factor receptor alpha gene. PT, preterm; T, term.

especially in males ${ }^{28}$. In addition, both increased and decreased digging and marble burying were noted as secondary ASD-like features related to emotional state and anxiety in several genetic ASD mouse models ${ }^{19,21,29-31}$.

Behavioral sex-linked differences between $\mathrm{C}$ and $\mathrm{plKO}$ mice can be detected even earlier in postnatal development. The ultrasonic vocalizations (USVs) emitted by the pups when separated from the dam (referred to as isolation calling response) were evaluated as a measure of the degree of aversive affective state and early communicative behavior. We found that plKO males produced fewer USV s than their littermate controls when separated from the dam (Fig. $3 j$ ). By contrast, no significant change was evidenced in the
plKO females (Fig. 3k). Deficient isolation calling response has been detected in different mouse models of $\mathrm{ASD}^{32}$, indicating that insufficient provision of placental ALLO to the fetal brain can lead to a wide array of autistic-like behaviors in the male progeny.

Cerebellar MBP correlates with ASD-like symptom severity. We calculated an autism severity composite index based on previously established scoring systems ${ }^{33}$. Behaviors were $\mathrm{z}$-standardized with higher values representing higher symptom severity. Individual behavioral scores were significantly higher in plKO males compared to $\mathrm{C}$ but not in females (Fig. 3d,e). Thus, the combined $\mathrm{z}$-scores yielded an elevated autism severity score in males (Fig. 3f). The 
relative frequency distribution of individual autism severity scores followed Gaussian distribution curves that differed in plKO males, which shifted to the right along the $x$ axis (Fig. 3g,h). Interestingly, some of the plKO mice exhibited scores similar to control mice, suggesting a spectrum of behavioral changes in male plKOs. This variability might arise from natural variations in placental progesterone and ALLO levels, variable deletion of akr1c14, mixed genetic background of the mice or subtle changes in hormone exposure due to in utero position of the fetus.

A significant positive correlation between autism symptom severity scores and cerebellar MBP levels was seen in plKO males (Fig. 3i). This finding is of particular interest knowing that transient WM hyperplasia, myelin thickening and/or over-expression of OL lineage and myelin markers such as olig2, $m b p$ and mag have been documented in the cerebellum of patients with $\operatorname{ASD}^{26,27}$ and several genetic ASD-like mouse model ${ }^{34,35}$. Together with the enhanced FA in the cerebellum-related tracts described above (Extended Data Fig. 4a), this correlation could reflect a short-range hyperconnectivity and/or a transient WM hyperplasia as seen in patients with autism ${ }^{26,36}$. The abnormal maturation speed of the OL lineage in plKO mice suggests dynamic, rather than uniform, changes of WM development, similar to the pattern seen across the lifespan of patients with $\mathrm{ASD}^{26}$. Overall, our placental mouse model recapitulates several characteristics of human studies, including the male greater vulnerability to perinatal brain injuries and $\mathrm{ASD}^{19,23,27,37}$.

ALLO or $\mathrm{GABA}_{\mathrm{A}}$ agonist rescues cerebellar and behavioral impairments. The therapeutic potential of ALLO to rescue cerebellar WM and behavioral abnormalities was then tested by administering ALLO (10 $\mathrm{mg} \mathrm{kg}^{-1}$ ) or vehicle (sesame oil) to dams carrying mixed litters at E15.5, the period of maximal difference of ALLO levels in the plKO mice compared to C. A single injection of ALLO during gestation prevented the cerebellar MBP up-regulation (Fig. 4a), normalized OL density in the cerebellar WM (Extended Data Fig. 10a) and reduced the autism severity score (Fig. 4b,c). To assess the specific contribution of $\mathrm{GABA}_{\mathrm{A}} \mathrm{R}$ signaling to this rescue, muscimol $\left(1 \mathrm{mg} \mathrm{kg}^{-1}\right)$, a selective $\mathrm{GABA}_{\mathrm{A}}$ agonist, was administered instead of ALLO. Muscimol injection resulted in similar molecular and behavioral rescue in plKO males (Fig. $4 \mathrm{a}-\mathrm{c}$ ), although the contribution of other minor, $\mathrm{GABA}_{\mathrm{A}}$-independent ALLO actions cannot be completely excluded ${ }^{38-40}$. Interestingly, a fraction of $\mathrm{C}$ mice exposed to ALLO treatment exhibited decreased social preference and subsequent higher autism score (Extended Data Fig. 10b,c), suggesting that excess ALLO exposure during fetal life might be detrimental. Interestingly, increased PROG exposure, along with other steroids, was previously linked to ASD risk in boys ${ }^{41}$. Our results support the potential therapeutic utility of ALLO administration during gestation if ALLO or its precursors are determined to be low (as might occur with chronic placental insufficiency) but, additionally, suggest the need to maintain fetal ALLO exposure within an appropriate physiological window.

Myelin genes are similarly altered in preterm infants and plKO mice. To further evaluate the translational potential of our findings, we asked whether preterm birth, a condition characterized by premature loss of placenta and a risk factor for $\mathrm{ASD}^{42}$, was associated with cerebellar myelin alterations similar to those seen in plKO mice. We examined 24 postmortem cerebellar vermises from term ( $>37$ weeks of gestation) and preterm $(<34$ weeks of gestation) infants who died at a mean corrected age of 6 weeks (Fig. 5a-c and Supplementary Table 3). Compelling similarities between our mouse model and human pathology were found in cerebellar MBP expression in preterm males and females (Fig. $5 \mathrm{~d}-\mathrm{h}, \mathrm{k}$ ) compared to corrected age-matched specimens. Furthermore, the migration downshift of MAG on western blot gels was observed in human preterm male cerebellum, similar to our findings in plKO males (Figs.
$2 \mathrm{a}$ and $5 \mathrm{~d}$ ), again suggesting accelerated maturation of myelin ${ }^{10}$. The higher levels of pdgfra- and cspg4-mRNA in the cerebellum of preterm males also suggest increased OPC proliferation (Fig. 5i,j,l), as temporarily seen in the plKO males (Extended Data Fig. 6e). This striking correlation between mice exposed to ALLO insufficiency and infants born preterm suggests that altered ALLO exposure might contribute to abnormal preterm brain development.

\section{Discussion}

Our study empirically defines a critical role for a specific placental hormone that can alter fetal brain development in late gestation with postnatal developmental consequences. Many neuroactive hormones are produced in the placenta in late gestation and might affect specific stages of neurogenesis and gliogenesis, neuronal migration and circuit formation. We focused on ALLO because this placental steroid hormone normally peaks in the second half of gestation, when placental insufficiency becomes evident and when preterm birth occurs. Interestingly, in severe pre-eclampsia, a pregnancy condition associated with both preterm birth and ASD, plasma ALLO levels in pregnant women are reduced, as compared to control group ${ }^{43}$, strengthening the potential link among ALLO, prematurity and ASD risk.

Many brain regions have been linked to autism, but structural and functional cerebellar abnormalities are among the most consistent findings ${ }^{44-46}$. Early cerebellar injury or dysfunction carries a high relative risk (up to $36 \times$ ) for ASD, exceeded only by genetic factors ${ }^{25}$. Both neuronal dysfunction, especially of cerebellar Purkinje cells, and WM changes have been linked to ASD phenotypes. Cerebellar transcriptome analysis highlighted WM-associated pathways as a top category of altered genes. Anatomical myelination changes, consistent with these transcriptomic findings, suggest that oligodendrogenesis and myelination significantly contribute to the observed outcomes. In our mouse model, Purkinje cell number appears normal (Extended Data Fig. 7j), but the contribution of Purkinje cell physiology to the myelin and behavioral phenotypes will be assessed in future studies. Additionally, the local specificity of changes will need to be further assessed. Motor and non-motor functions of the cerebellum are controlled by specific subdivisions-that is, lobules and modules-that are characterized by specific inputs and outputs, Purkinje cell firing frequency, degree of myelination and neurochemical signatures (for example, zebrin expression) ${ }^{47}$. Future studies addressing whether the increased myelin thickness in the cerebellum of plKO mice is global or restricted to functional zones, and whether it correlates with Purkinje cell firing frequency, might elucidate how placental ALLO insufficiency alters specific cerebellar functions that are linked to ASD.

Our experiments demonstrate a treatment-reversible link between specific placental ALLO insufficiency and sex-specific cerebellar myelination associated with an ASD-like phenotype. It is well documented that female and male brains might take different developmental trajectories in physiological and pathological conditions $^{48}$, but there is still limited mechanistic information on the underlying causes of sex differences ${ }^{49}$. The results we describe here suggest that, in the case of ALLO insufficiency and cerebellar myelination, this sex-linked divergence occurs primarily in the postnatal period. Furthermore, the sex-linked phenotypes of our mouse model parallel human pathological features, strengthening the translational value of our findings. These experiments suggest that the cerebellum is the primary brain region affected by the lack of placental ALLO in males. Other brain regions might be altered specifically in females, as our transcriptomic assessment suggests (Extended Data Fig. 5). Undoubtedly, such complex sexual dimorphism builds upon the interplay of a myriad of factors throughout prenatal and postnatal development. Further investigation of brain region-specific, sex-linked trajectories under normal and pathological conditions can pave the way for personalized risk 
assessment, diagnosis and treatment informed by patient sex and hormone levels.

Increased risk of ASD diagnosis after extremely preterm birth ${ }^{50}$, particularly in males, suggests that loss of placental hormones might contribute to specific human neurobehavioral outcomes in previously unanticipated ways. Our findings lay the groundwork for developing hormone replacement strategies to normalize the developmental milieu and prevent long-term impairments in neurobehavior.

\section{Online content}

Any methods, additional references, Nature Research reporting summaries, source data, extended data, supplementary information, acknowledgements, peer review information; details of author contributions and competing interests; and statements of data and code availability are available at https://doi.org/10.1038/ s41593-021-00896-4.

Received: 3 March 2021; Accepted: 23 June 2021; Published online: 16 August 2021

\section{References}

1. Kratimenos, P. \& Penn, A. A. Placental programming of neuropsychiatric disease. Pediatr. Res. 86, 157-164 (2019).

2. Hosie, A. M., Wilkins, M. E., da Silva, H. M. \& Smart, T. G. Endogenous neurosteroids regulate $\mathrm{GABA}_{\mathrm{A}}$ receptors through two discrete transmembrane sites. Nature 444, 486-489 (2006).

3. Belelli, D., Hogenkamp, D., Gee, K. W. \& Lambert, J. J. Realising the therapeutic potential of neuroactive steroid modulators of the GABA receptor. Neurobiol. Stress 12, 100207 (2020).

4. Schumacher, M. et al. Revisiting the roles of progesterone and allopregnanolone in the nervous system: resurgence of the progesterone receptors. Prog. Neurobiol. 113, 6-39 (2014).

5. Brunton, P. J., Russell, J. A. \& Hirst, J. J. Allopregnanolone in the brain protecting pregnancy and birth outcomes. Prog. Neurobiol. 113, 106-136 (2014).

6. Wenzel, P. L. \& Leone, G. Expression of Cre recombinase in early diploid trophoblast cells of the mouse placenta. Genesis 45, 129-134 (2007).

7. Marques, S. et al. Oligodendrocyte heterogeneity in the mouse juvenile and adult central nervous system. Science 352, 1326-1329 (2016).

8. Thakurela, S. et al. The transcriptome of mouse central nervous system myelin. Sci. Rep. 6, 25828 (2016).

9. Shaw, J. C., Palliser, H. K., Palazzi, K. \& Hirst, J. J. Administration of progesterone throughout pregnancy increases maternal steroids without adverse effect on mature oligodendrocyte immunostaining in the guinea pig. Reprod. Sci. 25, 395-405 (2018).

10. Marton, L. S. \& Stefansson, K. Developmental alterations in molecular weights of proteins in the human central nervous system that react with antibodies against myelin-associated glycoprotein. J. Cell Biol. 99, 1642-1646 (1984).

11. Chang, E. H. et al. The role of myelination in measures of white matter integrity: combination of diffusion tensor imaging and two-photon microscopy of CLARITY intact brains. Neuroimage 147, 253-261 (2017).

12. Baumann, N. \& Pham-Dinh, D. Biology of oligodendrocyte and myelin in the mammalian central nervous system. Physiol. Rev. 81, 871-927 (2001).

13. Chen, V. S. et al. Histology atlas of the developing prenatal and postnatal mouse central nervous system, with emphasis on prenatal days E7.5 to E18.5. Toxicol. Pathol. 45, 705-744 (2017).

14. van Tilborg, E. et al. Origin and dynamics of oligodendrocytes in the developing brain: Implications for perinatal white matter injury. Glia $\mathbf{6 6}$, 221-238 (2018).

15. Hamilton, N. B. et al. Endogenous GABA controls oligodendrocyte lineage cell number, myelination, and CNS internode length. Glia 65, 309-321 (2017).

16. Zonouzi, M. et al. GABAergic regulation of cerebellar NG2 cell development is altered in perinatal white matter injury. Nat. Neurosci. 18, 674-682 (2015).

17. Sathyanesan, A., Kundu, S., Abbah, J. \& Gallo, V. Neonatal brain injury causes cerebellar learning deficits and Purkinje cell dysfunction. Nat. Commun. 9 , 3235 (2018).

18. Grasselli, G. et al. SK2 channels in cerebellar Purkinje cells contribute to excitability modulation in motor-learning-specific memory traces. PLoS Biol. 18, e3000596 (2020).

19. Piochon, C. et al. Cerebellar plasticity and motor learning deficits in a copy-number variation mouse model of autism. Nat. Commun. 5, 5586 (2014).
20. Vinueza Veloz, M. F. et al. Cerebellar control of gait and interlimb coordination. Brain Struct. Funct. 220, 3513-3536 (2015).

21. Kindregan, D., Gallagher, L. \& Gormley, J. Gait deviations in children with autism spectrum disorders: a review. Autism Res Treat. 2015, 741480 (2015).

22. Scholz, J., Niibori, Y., Frankland, P. W. \& Lerch, J. P. Rotarod training in mice is associated with changes in brain structure observable with multimodal MRI. Neuroimage 107, 182-189 (2015).

23. Rothwell, P. E. et al. Autism-associated neuroligin-3 mutations commonly impair striatal circuits to boost repetitive behaviors. Cell 158, 198-212 (2014).

24. Stoodley, C. J. et al. Altered cerebellar connectivity in autism and cerebellar-mediated rescue of autism-related behaviors in mice. Nat. Neurosci. 20, 1744-1751 (2017)

25. Wang, S. S., Kloth, A. D. \& Badura, A. The cerebellum, sensitive periods, and autism. Neuron 83, 518-532 (2014).

26. Courchesne, E. et al. Unusual brain growth patterns in early life in patients with autistic disorder: an MRI study. Neurology 57, 245-254 (2001).

27. Zeidan-Chulia, F. et al. Up-regulation of oligodendrocyte lineage markers in the cerebellum of autistic patients: evidence from network analysis of gene expression. Mol. Neurobiol. 53, 4019-4025 (2016).

28. Silverman, J. L., Yang, M., Lord, C. \& Crawley, J. N. Behavioural phenotyping assays for mouse models of autism. Nat. Rev. Neurosci. 11, 490-502 (2010).

29. Gallo, I. et al. Formyl peptide receptor as a novel therapeutic target for anxiety-related disorders. PLoS ONE 9, e114626 (2014).

30. Kim, H., Lim, C. S. \& Kaang, B. K. Neuronal mechanisms and circuits underlying repetitive behaviors in mouse models of autism spectrum disorder. Behav. Brain Funct. 12, 3 (2016).

31. Lugo, J. N. et al. Deletion of PTEN produces autism-like behavioral deficits and alterations in synaptic proteins. Front. Mol. Neurosci. 7, 27 (2014).

32. Premoli, M. et al. Specific profile of ultrasonic communication in a mouse model of neurodevelopmental disorders. Sci. Rep. 9, 15912 (2019).

33. El-Kordi, A. et al. Development of an autism severity score for mice using Nlgn4 null mutants as a construct-valid model of heritable monogenic autism. Behav. Brain Res. 251, 41-49 (2013).

34. Fraser, M. M., Bayazitov, I. T., Zakharenko, S. S. \& Baker, S. J. Phosphatase and tensin homolog, deleted on chromosome 10 deficiency in brain causes defects in synaptic structure, transmission and plasticity, and myelination abnormalities. Neuroscience 151, 476-488 (2008).

35. Pacey, L. K. et al. Delayed myelination in a mouse model of fragile X syndrome. Hum. Mol. Genet 22, 3920-3930 (2013).

36. Uddin, L. Q., Supekar, K. \& Menon, V. Reconceptualizing functional brain connectivity in autism from a developmental perspective. Front Hum. Neurosci. 7, 458 (2013).

37. Dachew, B. A., Mamun, A., Maravilla, J. C. \& Alati, R. Pre-eclampsia and the risk of autism-spectrum disorder in offspring: meta-analysis. Br. J. Psychiatry 212, 142-147 (2018).

38. Guennoun, R. Progesterone in the brain: hormone, neurosteroid and neuroprotectant. Int. J. Mol. Sci. 21, 5271 (2020).

39. Pang, Y., Dong, J. \& Thomas, P. Characterization, neurosteroid binding and brain distribution of human membrane progesterone receptors $\delta$ and $\varepsilon$ ( $\mathrm{mPR} \delta$ and $\mathrm{mPR} \varepsilon$ ) and $\mathrm{mPR} \delta$ involvement in neurosteroid inhibition of apoptosis. Endocrinology 154, 283-295 (2013).

40. Thomas, P. \& Pang, Y. Anti-apoptotic actions of allopregnanolone and ganaxolone mediated through membrane progesterone receptors (PAQRs) in neuronal cells. Front. Endocrinol. (Lausanne) 11, 417 (2020).

41. Baron-Cohen, S. et al. Foetal oestrogens and autism. Mol. Psychiatry 25, 2970-2978 (2020)

42. Agrawal, S., Rao, S. C., Bulsara, M. K. \& Patole, S. K. Prevalence of autism spectrum disorder in preterm infants: a meta-analysis. Pediatrics 142, e20180134 (2018)

43. Hertig, A. et al. Steroid profiling in preeclamptic women: evidence for aromatase deficiency. Am. J. Obstet. Gynecol. 203, 477 e471-477 (2010).

44. Becker, E. B. \& Stoodley, C. J. Autism spectrum disorder and the cerebellum. Int. Rev. Neurobiol. 113, 1-34 (2013).

45. Fatemi, S. H. et al. Consensus paper: pathological role of the cerebellum in autism. Cerebellum 11, 777-807 (2012).

46. Rogers, T. D. et al. Is autism a disease of the cerebellum? An integration of clinical and pre-clinical research. Front. Syst. Neurosci. 7, 15 (2013).

47. De Zeeuw, C. I. \& Ten Brinke, M. M. Motor learning and the cerebellum. Cold Spring Harb. Perspect. Biol. 7, a021683 (2015).

48. McCarthy, M. M. Sex differences in the developing brain as a source of inherent risk. Dialogues Clin. Neurosci. 18, 361-372 (2016).

49. McCarthy, M. M., Arnold, A. P., Ball, G. F., Blaustein, J. D. \& De Vries, G. J Sex differences in the brain: the not so inconvenient truth. J. Neurosci. 32, 2241-2247 (2012)

50. Limperopoulos, C. Autism spectrum disorders in survivors of extreme prematurity. Clin. Perinatol. 36, 791-805 (2009).

Publisher's note Springer Nature remains neutral with regard to jurisdictional claims in published maps and institutional affiliations. 


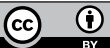

Open Access This article is licensed under a Creative Commons Attribution 4.0 International License, which permits use, sharing, adaptation, distribution and reproduction in any medium or format, as long as you give appropriate credit to the original author(s) and the source, provide a link to the Creative Commons license, and indicate if changes were made. The images or other third party material in this article are included in the article's Creative Commons license, unless indicated otherwise in a credit line to the material. If material is not included in the article's Creative Commons license and your intended use is not permitted by statutory regulation or exceeds the permitted use, you will need to obtain permission directly from the copyright holder. To view a copy of this license, visit http://creativecommons. org/licenses/by/4.0/.

(C) The Author(s) 2021 


\section{Methods}

Mice. All the procedures on experimental animals were performed in accordance with the protocol approved by the Institutional Animal Care and Use Committee at Children's National Medical Center (protocol no. 30534 (PI: Penn)) and at Columbia University Medical Center (protocol nos. AC-AABE 6553 (PI: Penn) and AC-AABF 5550 (PI: Yang)). Mice were housed on a 12-h light/dark cycle and had ad libitum access to food and water.

The akrlc14-floxed mouse was constructed with inGenious Targeting Laboratories. The construct targeted a 3.15-kb region that includes exons 7-9 of akr1c14, with a $5^{\prime}$ homology arm extending to the position of a LoxP-FRT flanked Neo cassette and a $3^{\prime}$ homology arm extending to a single LoxP site just $5^{\prime}$ of exon 10 (Fig. 1b). The targeting vector was electroporated into embryonic stem cells, which were cloned and screened. Confirmed clones were micro-injected into C57Bl/6 blastocysts. The Neo cassette was later removed using flipase. The resulting mice are designated as the akrlc14-floxed line. akr1c14 $4^{\mathrm{t} / \mathrm{wt}}$ and akr1c14 $4^{\mathrm{t} /}$ ${ }^{\mathrm{fl}}$ mice appear phenotypically normal. The final placental $3 \alpha-\mathrm{HSD}$ knockout was generated by successive crosses of progenitor akrlc14-floxed mice and Cyp19a-Cre mice (generous gift of G. Leone), which express Cre exclusively in placental trophoblast cells ${ }^{6}$. Mouse, unlike human, does not express endogenous placental Cyp19a, which codes for aromatase, but the human Cyp19a promotor effectively drives placenta-specific Cre expression ${ }^{6}$. Cyp19a-Cre females were crossed to homozygous akrlc1 $4^{\mathrm{t} / \mathrm{f}}$ males. Female offspring of these crosses that were positive for Cre with two floxed alleles were used as the dams of experimental mice. These akr1c14/1/1/ females carrying Cyp19a-Cre were crossed to akr1c14 $4^{\mathrm{A} / \mathrm{l}}$ males, so that half of resulting offspring are both homozygous akr1c1 $4^{\mathrm{fl} / \mathrm{l}}$ and positive for placental Cre. In tests of recombination specificity (Fig. 1e), the presence of both LoxP sites (indicating un-recombined DNA) was ascertained using a primer set, including a forward primer homologous to a region $5^{\prime}$ of exon 7 , and a reverse primer incorporating the $3^{\prime}$ LoxP site between exons 9 and 10 . This primer pair was specific to the floxed allele due to the inclusion of the LoxP site in the reverse primer sequence. It did not amplify the wild-type allele. The presence of the recombined LoxP site was ascertained using a primer set, which flanks exon 10 such that the recombined allele ( $164 \mathrm{bp}$ in length) amplifies, whereas the intact allele is too long to amplify (3,396bp). These mice in which akr1c14 is deleted specifically in the placenta are akr1c14 $4^{\mathrm{Cyp} 19 \mathrm{a}-/-}$, designated as akr1c14 ${ }^{\mathrm{Cyp} 19 \mathrm{a}} \mathrm{KO}$ (plKO) for simplicity. An additional half of offspring are negative for placental Cre and designated as controls $(\mathrm{C})$.

Cyp19a-Cre transgenic mice were crossed with ROSA26-YFP mice (The Jackson Laboratory, no. 006148) to obtain Cyp19a-Cre:R26R-EYFP littermates.

Human samples. Human cerebellar vermes were obtained from the National Institutes of Health (NIH) NeuroBioBank (request ID no. 709). Donors consisted of 6-week-old term infants (average age) and corrected age-matched preterm infants, excluding those with major congenital anomalies or known genetic diagnoses and those with meningitis or stroke as cause of death. Sex, gestational weeks, absolute age (postnatal age) and corrected age are shown in Supplementary Table 3. Frozen tissues were preserved at $-80^{\circ} \mathrm{C}$.

Drug injections. Pregnant dams received one intraperitoneal injection of allopregnanolone (Tocris Bioscience, no. 3653) diluted in sesame oil (vehicle; Sigma-Aldrich, no. S3547) at E15.5 at a dose of $10 \mathrm{mg} \mathrm{kg}^{-1}$ of body weight. Muscimol (Tocris, no. 0289) was diluted in saline solution and injected intraperitoneally in pregnant mice at E15.5 $\left(1 \mathrm{mg} \mathrm{kg}^{-1}\right)$. Allopregnanolone and muscimol injections were done in the morning, during sleep time, to limit the effect of the drug-induced sedation on dams' activity. Doses were chosen based on prior pharmacological studies in mice ${ }^{51,52}$, and the timing was an empirical choice based on akrlc14 gene expression peak at E14.5 (Fig. 1a). BrdU (50 $\left.\mathrm{mg} \mathrm{kg}^{-1}\right)$ dissolved in saline solution was injected intraperitoneally in dams at E15.5.

Mass spectrometry. Steroid extraction. Pregnenolone (PREG), PROG, ALLO, epiallopregnanolone (EPIALLO), ALLOTHDOC and $3 \alpha 5 \alpha$-tetrahydrotestosterone (3 $\alpha 5 \alpha-$ THT) were identified and quantified simultaneously in individual tissues by gas chromatography-tandem mass spectrometry (GC-MS/MS) as previously described ${ }^{53}$. Placental $(65-185 \mathrm{mg})$ and fetal brain $(61-130 \mathrm{mg})$ tissues of male and female wild-type and plKO mice at E17.5 were weighed and stored at $-20^{\circ} \mathrm{C}$ until GC-MS/MS analysis. Briefly, steroids were first extracted from placentas, and brains with 10 volumes of methanol $(\mathrm{MeOH})$ and the following internal standards were added to the extracts for steroid quantification: $2 \mathrm{ng}$ of epietiocholanolone for PREG, ALLO, EPIALLO, ALLOTHDOC and $3 \alpha 5 \alpha$-THT and $2 \mathrm{ng}$ of ${ }^{13} \mathrm{C}_{3}$-PROG for PROG. Samples were purified and fractionated by solid-phase extraction with the recycling procedure ${ }^{54}$. The extracts were dissolved in $1 \mathrm{ml}$ of $\mathrm{MeOH}$ and applied to the $\mathrm{C} 18$ cartridge $(500 \mathrm{mg}, 6 \mathrm{ml}$, International Sorbent Technology), followed by $5 \mathrm{ml}$ of $\mathrm{MeOH} / \mathrm{H}_{2} \mathrm{O}(85 / 15, \mathrm{vol} / \mathrm{vol})$. The flow-through, containing the free steroids, was collected and dried. After a previous re-conditioning of the same cartridge with $5 \mathrm{ml}$ of $\mathrm{H}_{2} \mathrm{O}$, the dried samples were dissolved in $\mathrm{MeOH} / \mathrm{H}_{2} \mathrm{O}(2 / 8$, $\mathrm{vol} / \mathrm{vol}$ ) and re-applied. The cartridge was then washed with $5 \mathrm{ml}$ of $\mathrm{H}_{2} \mathrm{O}$ and $5 \mathrm{ml}$ of $\mathrm{MeOH} / \mathrm{H}_{2} \mathrm{O}(1 / 1, \mathrm{vol} / \mathrm{vol})$, and unconjugated steroids were eluted with $5 \mathrm{ml}$ of $\mathrm{MeOH} / \mathrm{H}_{2} \mathrm{O}(9 / 1, \mathrm{vol} / \mathrm{vol})$. The fraction containing the unconjugated steroids was then filtered and further purified by high-performance liquid chromatography
(HPLC). The HPLC system is composed of a WPS-3000SL analytical autosampler and an LPG-3400SD quaternary pump gradient coupled with a SR-3000 fraction collector (Thermo Fisher Scientific). The HPLC separation was achieved with a LiChrosorb Diol column $(25 \mathrm{~cm}, 4.6 \mathrm{~mm}, 5 \mu \mathrm{m})$ in a thermostated block at $30^{\circ} \mathrm{C}$ The column was equilibrated in a solvent system of $90 \%$ heptane and $10 \%$ of a mixture composed of heptane/isopropanol $(85 / 15, \mathrm{vol} / \mathrm{vol})$. Elution was performed at a flow rate of $1 \mathrm{ml} \mathrm{min}^{-1}$, first $90 \%$ heptane and $10 \%$ of heptane/isopropanol $(85 / 15, \mathrm{vol} / \mathrm{vol})$ for $15 \mathrm{~min}$, and then with a linear gradient to $100 \%$ acetone in $2 \mathrm{~min}$. The column was washed with acetone for $15 \mathrm{~min}$. Steroids were collected in the time range of 15-29 min and were derivatized with $25 \mu \mathrm{l}$ of HFBA and $25 \mu \mathrm{l}$ of anhydrous acetone for $1 \mathrm{~h}$ at $20^{\circ} \mathrm{C}$. Samples were dried under a stream of nitrogen and resuspended in heptane.

GC-MS/MS analysis. GC-MS/MS analysis of the biological extracts was performed using an AI 1310 autosampler, a Trace 1310 gas chromatograph and a TSQ 8000 mass spectrometer (Thermo Fisher Scientific). Injection was performed in the splitless mode at $250^{\circ} \mathrm{C}$ ( $1 \mathrm{~min}$ of splitless time), and the temperature of the gas chromatograph oven was initially maintained at $80^{\circ} \mathrm{C}$ for $1 \mathrm{~min}$ and ramped between $80^{\circ} \mathrm{C}$ and $200^{\circ} \mathrm{C}$ at $20^{\circ} \mathrm{C} \mathrm{min}^{-1}$ and then ramped to $300^{\circ} \mathrm{C}$ at $5^{\circ} \mathrm{C}$ $\mathrm{min}^{-1}$ and finally ramped to $350^{\circ} \mathrm{C}$ at $30^{\circ} \mathrm{C} \mathrm{min}^{-1}$. The helium carrier gas flow was maintained constant at $1 \mathrm{ml} \mathrm{min}^{-1}$ during the analysis. The transfer line and ionization chamber temperatures were $300^{\circ} \mathrm{C}$ and $200^{\circ} \mathrm{C}$, respectively. Electron impact ionization was used for mass spectrometry with ionization energy of $70 \mathrm{eV}$, and GC-MS/MS analysis was performed in multiple reaction monitoring mode with argon as the collision gas. GC-MS/MS signals were evaluated using a computer workstation by means of the software Excalibur, release 3.0 (Thermo Fisher Scientific). Identification of steroids was supported by their retention time and two or three transitions. Quantification was performed according to the more abundant transition with a previously established calibration curve. The range of the limit of detection was roughly $0.5-20 \mathrm{pg}$ according to the steroid structure. The GC-MS/MS analytical procedure was fully validated in terms of accuracy, reproducibility and linearity in mouse brain ${ }^{53}$

$3^{\prime}$ mRNA sequencing. Tissue collection and RNA extraction: Cerebellum, bilateral cerebral cortices and bilateral hippocampi from $\mathrm{C}$ and $\mathrm{plKO}$ mice (males and females) at P30 (three animals per group) were dissected and flash-frozen. Tissue homogenization and total RNA extraction were performed using the mirVana Isolation Kit (Thermo Fisher Scientific, no. AM1560) according to the manufacturer's instructions.

Library preparation and sequencing for $m R N A$. The cDNA libraries were prepared using the QuantSeq 3' mRNA-Seq Library Prep Kit FWD for Illumina (Lexogen) as per the manufacturer's instructions. Briefly, total RNA was reverse transcribed using oligo $(\mathrm{dT})$ primers. The second cDNA strand was synthesized by random priming, in a manner that DNA polymerase is efficiently stopped when reaching the next hybridized random primer; therefore, only the fragment closed to the $3^{\prime}$ end was captured for indexed adapter ligation and PCR amplification. The processed libraries were assessed for its size distribution and concentration using BioAnalyzer High Sensitivity DNA Kit (Agilent Technologies, no. 5067-4626). Pooled libraries were diluted to $2 \mathrm{nM}$ in EB buffer (Qiagen, no. 19086) and then denatured using the Illumina protocol. The libraries were pooled and diluted to $3 \mathrm{nM}$ using $10 \mathrm{mM}$ Tris- $\mathrm{HCl}, \mathrm{pH}$ 8.5, and then denatured using the Illumina protocol. The denatured libraries were diluted to $10 \mathrm{pM}$ by pre-chilled hybridization buffer and loaded onto a TruSeq v2 Rapid flow cell on an Illumina HiSeq 2500 and run for 50 cycles using a single-read recipe according to the manufacturer's instructions. De-multiplexed sequencing reads were generated using Illumina bcl2fastq (release version 2.18.0.12) allowing no mismatches in the index read.

Data analysis. After the quality and polyA trimming by BBDuk ${ }^{55}$ and alignment by HISAT2 (version 2.1.0) ${ }^{56}$, read counts were calculated using HTSeq ${ }^{57}$ by supplementing Ensembl gene annotation (GRCm38.78). DE analysis and MA plots were done using TCC R package (version 1.12.1, https://bioconductor. riken.jp/packages/3.3/bioc/manuals/TCC/man/TCC.pdf) ${ }^{58}$. After getting DEGs, hierarchical clustering of DEGs as a heat map was performed using Partek Genomics Suite (version 6.6, http://www.partek.com/partek-genomics-suite/) (Partek). No outlier was identified in our biological replicates, as indicated by principal component analysis and scatter matrices. DEGs met the following guidelines: $P<0.05$; fragments per kilobase of transcript per million mapped reads values $>1$; and fold change $>1.5$. The limits of these cutoffs were validated by RT-PCR on samples from other mouse cohorts. IPA was used to identify the top biological functions and disease processes that were differentially regulated. This software is based on the Ingenuity Pathway Knowledge Base for genetic interaction, which derives from the scientific literature, each network connection being supported by previous publications $s^{59}$. To determine and visualize the degree of gene overlaps in datasets, Venn analysis was performed using Venny 2.1 (http:// bioinfogp.cnb.csic.es/tools/venny/).

RT-PCR. Tissues were homogenized in TRIzol Reagent (Thermo Fisher Scientific); total RNA was extracted with the RNeasy Mini Kit (Qiagen, no. 74104). 
Next, $1 \mu \mathrm{g}$ of RNA was used to make cDNA with the iScript cDNA Synthesis Kit (Bio-Rad, no. 1708891). All primer pairs were designed and validated in-house for efficiency and specificity. RT-PCR experiments were performed on cDNA samples in the presence of PowerUp SYBR Green Master Mix (Thermo Fisher Scientific, no. 1725271) with specific primers at $100 \mathrm{nM}$ using the CFX96 Touch Real-Time PCR Detection System (Bio-Rad). The cDNA-generated signals for target genes were internally corrected with phosphoglycerate kinase $1(p g k 1)$ in mouse postnatal brains, tyrosine 3-monooxygenase/tryptophan 5-monooxygenase activation protein zeta ( $y$ whaz) in mouse placentas and embryonic brains or cytochrome $\mathrm{C}$ $(c y c 1)$ in human tissues. The regulation fold changes were determined with the $2^{-\Delta \Delta \mathrm{Cq}}$ method $^{60}$

MRI. Brain preparation. Brains left within the skull were prepared for MRI using the following method. Mice were anesthetized and intracardially perfused at a rate of $1 \mathrm{ml} \mathrm{min}^{-1}$, with $30 \mathrm{ml}$ of $0.1 \mathrm{M}$ PBS containing $10 \mathrm{U} / 100 \mathrm{ml}$ heparin (Sigma-Aldrich) and $2 \mathrm{mM}$ ProHance (gadolinium contrast agent, Bracco Diagnostics) followed by $30 \mathrm{ml}$ of $4 \%$ paraformaldehyde (PFA) and $2 \mathrm{mM}$ ProHance. After perfusion, the mice were decapitated, and the skin and the lower jaw were removed. Brains in skulls were post-fixed in 4\% PFA and $2 \mathrm{mM}$ ProHance at $4{ }^{\circ} \mathrm{C}$ overnight and then kept in $0.1 \mathrm{M}$ PBS containing $2 \mathrm{mM}$ ProHance and $0.02 \%$ sodium azide for at least 1 month before MRI scanning ${ }^{61,62}$.

DTI acquisition. A multi-channel 7-Tesla MRI scanner (Agilent) was used to image the brains. For DTI Imaging, 12 brains were scanned in parallel using a 3D diffusion-weighted fast spin echo $\operatorname{scan}^{63,64}$. Parameters for the DTI sequence were as follows: TR of $270 \mathrm{~ms}$, echo train length of 6 , first TE of $32 \mathrm{~ms}$ and a TE of $10 \mathrm{~ms}$ for the remaining five echoes, 1 average, field of view of $14 \mathrm{~mm} \times 14 \mathrm{~mm} \times 25 \mathrm{~mm}$ and a matrix size of $180 \times 180 \times 324$, resulting in an image with $78-\mu \mathrm{m}$ isotropic voxels. Five $b=0 \mathrm{~s} \mathrm{~mm}^{-2}$ images and 30 high $b$-value $\left(b=2,147 \mathrm{~s} \mathrm{~mm}^{-2}\right)$ images in 30 different directions were acquired, using the Jones 30 scheme $^{65}$. Total imaging time was approximately $12 \mathrm{~h}$. The FSL software package (FMRIB) was used to generate FA and mean diffusivity (MD) maps for each brain sample.

$M R I$ registration and analysis. To assess any changes to the mouse brains due to genotype and sex, the $b=0 \mathrm{~s} \mathrm{~mm}^{-2}$ images were registered linearly ( 6 followed by 12 parameter) and non-linearly together. A combination of mni_autoreg tools ${ }^{66}$ and ANTs (advanced normalization tools) ${ }^{67}$ were used to perform the registrations. A population atlas, representing the average anatomy of the study samples, was created when all scans were resampled with an appropriate transform. The final registration results are the individual images deformed into alignment with one another in an unbiased manner. The analysis of the individual DTI parameters compare intensity differences of FA and MD between genotype and sex. Warping an available classified MRI mouse brain atlas onto the population average allows for significant differences in the diffusion measures of segmented structures to be calculated ${ }^{68-71}$

Histology. Section preparation and staining. Animals were anesthetized using isoflurane (Isothesia, Henry Schein Animal Health) and transcardially perfused with $20 \mathrm{ml}$ of $1 \times$ PBS followed by $30 \mathrm{ml}$ of $4 \%$ PFA, as previously described. Brains were post-fixed for $24 \mathrm{~h}$ in $4 \%$ PFA and cryoprotected in $20 \%$ sucrose in PBS. Serial $40-\mu \mathrm{m}$-thick sagittal brain/cerebellar sections were then collected using a sliding microtome. Placentas were collected at E17.5 after deep anaesthesia of the dams, drop-fixed in 4\% PFA and cryoprotected in $30 \%$ sucrose. Frozen $20-\mu \mathrm{m}$ cross-sections were obtained with a cryostat. Immunohistochemistry on brain or placenta sections was performed using the following antibodies: rabbit anti-MBP (Abcam, no. ab40390, 1:500), mouse anti-APC clone CC1 (EMD Millipore, no. MABC20, 1:500), rabbit anti-Olig2 (Abcam, no. ab9610, 1:500), mouse anti-neuronal nuclei (NeuN) (Millipore, no. MAB377, 1:500), goat anti-NeuroD1 (RD Systems, no. AF2746, 1:500), rabbit anti-calbindin (Swant, no. CB38, 1:1,000), rat anti-mouse PDGFR $\alpha$ (CD140a; BD Biosciences, 17-1401-81, 1:500) and chicken anti-GFP (Abcam, no. ab13970, 1:500). Sections were incubated with primary antibodies overnight at $4{ }^{\circ} \mathrm{C}$ containing $0.3 \%$ Triton and $10 \%$ normal donkey serum. Sections were then incubated with secondary antibodies (1:500) together with DAPI (Invitrogen, no. D1306, 1:1,000) for $2 \mathrm{~h}$ at room temperature. FluoroMyelin Green (Invitrogen, F34651, 1:500) was also added at this time for those sections. Secondary antibodies were used as follows: donkey anti-mouse Alexa-488 (Invitrogen, A-21202), donkey anti-rabbit Alexa-488 (Invitrogen, A-21206), donkey anti-mouse Alexa-555 (Invitrogen, A-31570), donkey anti-rabbit Alexa-555 (Invitrogen, A-31572), donkey anti-mouse Alexa-647 (Invitrogen, A-31571) and donkey anti-rabbit Alexa-647 (Invitrogen, A-31573). Finally, floating sections were mounted and cover-slipped using ProLong Gold Antifade Mountant (Thermo Fisher Scientific, P36930) before imaging.

In situ hybridization. For analysis of akrlc14-mRNA, E17.5 placental tissue was drop-fixed overnight in $4 \%$ PFA and then transferred through a sucrose gradient of $10 \%, 20 \%$ and $30 \%$ over $3 \mathrm{~d}$. Tissue was cross-sectioned at $12 \mu \mathrm{m}$ and assayed for akr1c14-mRNA using the Advanced Cell Diagnostics RNAScope system and an ACD probe designed to target the 621-1,989 region of akr1c14 (NM_134072.1).
Fluorescence imaging and cell counting. Placenta cross-sections and whole brain or cerebellum sagittal sections were imaged using a virtual slide microscope using cellSens version 2.3 (VS 120, Olympus Life Science) under a $\times 20$ objective. High-magnification images were taken under a confocal microscope with LAS X Life Science Microscope software version 2.7 (TCS SP8, Leica Microsystems). $z$-stack images were acquired with a step size of $1.55 \mu \mathrm{m}$ and viewed using NIH ImageJ 1.53c (http://imagej.nih.gov/ij). The different cerebellar layers were delineated manually using the freehand selection tool in ImageJ. The density of Olig2-, CC1-, PDGFR $\alpha$ - and BrdU-positive cells was estimated using Stereo Investigator (MBF Bioscience). After delineating the cerebellar WM using the freehand selection tool on corresponding DAPI images, immunopositive cells were manually counted under the $\times 40$ objective (Zeiss Axio Imager M2) using the optical fractional as a probe in grids randomly distributed on and covering 30\% of the cerebellar WM. Cell densities were then calculated by using the individual WM area values and the mean measured section thickness. The linear density of Purkinje cells (Purkinje cells per millimeter) was determined in the lobule VI-VII by drawing a freehand line through the center of the cell bodies.

Electron microscopy. Sample preparation. P30 mice were anesthetized with a 10:1 mixture of ketamine/xylazine in saline vehicle such that each mouse receives $100 \mathrm{mg} \mathrm{kg}^{-1}$ of ketamine and $10 \mathrm{mg} \mathrm{kg}^{-1}$ of xylazine, respectively. Under deep anaesthesia, mice were perfused with $20 \mathrm{ml}$ of $0.12 \mathrm{M}$ cacodylate buffer followed by $30 \mathrm{ml}$ of fixative made of $2.5 \%$ glutaraldehyde and 1\% PFA (electron microscopy grade, EMS nos. 16221 and 15713) in cacodylate buffer ( $\mathrm{pH} 7.4)$. Brains were removed and post-fixed for $1 \mathrm{~h}$ in the same fixative. Next, 300- $\mu$ m-thick sagittal slices were made using a vibratome (VT 1000S, Leica Biosystems). Slices were post-fixed with $1 \%$ osmium tetroxide in $0.12 \mathrm{M}$ cacodylate buffer for $2 \mathrm{~h}$ at room temperature and 'en block' stained with $1 \%$ uranyl acetate in $0.1 \mathrm{M}$ acetate buffer overnight at room temperature. After several washes in acetate buffer, slices were dehydrated by passing them through increasing concentrations of ethyl alcohol (up to $100 \%)$. Slices were then progressively infiltrated in epoxy resin and placed for $48 \mathrm{~h}$ at $60^{\circ} \mathrm{C}$ for resin polymerization. Then, 100 -nm-thick sagittal ultrasections were performed using an ultramicrotome (Ultracut UC7, Leica Biosystems) through the whole cerebellum (+ brainstem) or the whole anterior brain (for corpus callosum analyses).

Image acquisition. Large ultrathin sections absorbed on silicon were observed with a FEI Helios NanoLab 660 FIBSEM field emission scanning electron microscope (FEI, Thermo Fisher Scientific) using high-resolution immersion mode and equipped with a solid-state concentric ring (insertable) back-scattering electron detector. Image acquisition was done using MAPS Software version 3.7 (Thermo Fisher Scientific). Low-magnification images $(\times 600)$ were first taken to delineate our regions of interest (vermal inter-lobule 6-7 of the cerebellum). Then, high magnifications of two-dimensional image registration $(\times 20,000-50,000)$ were taken using $4 \mathrm{kV} / 0.2 \mathrm{nAmp}$ landing electron beam.

Myelin thickness measurement. The outer diameter (including myelin sheath) and inner diameter of at least 400 randomly selected myelinated axons were measured on one ultrathin section per animal (three animals per group). The g-ratio (equal to the ratio of the inner-to-outer diameter of a myelinated axon) of each axon was calculated. Scatter plots of the axon caliber and g-ratios were then analyzed. The slopes and elevations of the linear regressions from $\mathrm{C}$ and plKO mice were compared.

Western blots. Whole mouse cerebellums and human vermal samples were homogenized in $250 \mu \mathrm{l}$ of radioimmunoprecipitation assay lysis buffer consisting of (in mM) 50 Tris- $\mathrm{HCl}, \mathrm{pH} 7.4,150 \mathrm{NaCl}, 2$ EDTA, $50 \mathrm{NaF}, 1 \mathrm{Na}_{3} \mathrm{VO}_{4}, 1 \%$ Triton $\mathrm{X}-100,0.1 \%$ SDS, $0.5 \%$ Na-deoxycholate and a Protease/Phosphatase Inhibitor Cocktail (Santa Cruz Biotechnology). After centrifugation at $14,000 \mathrm{~g}$ for $10 \mathrm{~min}$, protein concentration was determined using Bradford protein assay kit (Bio-Rad). Sample total proteins were resolved by sodium dodecyl sulfate-polyacrylamide gel electrophoresis using 10\% Bis-Tris precast gel (Thermo Fisher Scientific) and transferred to polyvinylidene fluoride membranes. Membranes were incubated with blocking buffer consisting of $4 \%$ non-fat milk in $1 \%$ Tween-20 in Tris-buffered saline (TBS-T) for $1 \mathrm{~h}$, followed by overnight incubation at $4{ }^{\circ} \mathrm{C}$ with one of the following primary antibodies diluted in 3\% BSA TBST-T: rabbit anti-MBP (Abcam, no. ab40390, 1:500), rabbit anti-MAG (Thermo Fisher Scientific, no. PA5-79620, 1:500), rabbit anti-MOG (Abcam, 1:500), mouse anti-cofilin-1 (Cfl1) (Santa Cruz Biotechnology, no. sc-53934, 1:500), mouse anti-prosaposin (Santa Cruz Biotechnology, no. sc-390184, 1:500), rabbit anti-hnRNPא (R332) (Cell Signaling Technology, no. 4675, 1:500) and rabbit anti-GAPDH (Cell Signaling Technology, no. 5174, 1:2,000). After three washes with TBS-T, membranes were incubated with horseradish peroxide-conjugated secondary antibodies (Jackson ImmunoResearch, 1:2,000), and protein bands were visualized using the chemiluminescent ECL detection system (Bio-Rad) according to the manufacturer's instruction. Signal intensities of protein bands were quantified using Image J software (http://rsb.info.nih.gov/ij/) and normalized with GAPDH as an internal control. 
Behavioral testing. Tests were performed on 30- and 60-d-old mice. No more than three behavioral tests were done on each mouse, accordingly to our Institutional Animal Care and Use Committee recommendations. A minimal resting period of 3 $\mathrm{d}$ was allowed between two consecutive tests.

Pup ultrasonic vocalizations. Mouse pups emit USVs when separated from the dam. This innate behavior is essential for eliciting maternal care behaviors that the pups need to survive. Separation-induced vocalizations were tested on postnatal days 4, 6,8 and 11, with vocalizations declining drastically after P12. USVs were recorded for $3 \mathrm{~min}$, and the number of calls was tracked using Avisoft-SASLab Pro software (Avisoft Bioacoustics).

Erasmus ladder. The Erasmus ladder (Noldus) is a fully automated system allowing for the assessment of baseline gait and locomotor coordination and associative cerebellar learning. The instrument consists of a horizontal ladder with $2 \times 37$ pressure-sensitive rungs for both the left and right sides, stationed between two shelter boxes. Each shelter is equipped with a white LED spotlight and pressurized air outlet serving as cues to signal time to departure from shelters. As the mouse is prompted to move, pressure sensors continuously monitor and analyze the walking pattern of the mouse in real time, sending data to the computer system, which, in turn, adjusts air pressure, calculates interventions and predicts future steps. Through this prediction, the software can also be programmed to move rungs mid-trial by high-speed pneumatic slides to create an obstacle. Mice were trained with 42 runs per day for four successive days without obstacles. Missteps, trial times and jumps were measured for each run. After training, cerebellar associative motor learning was assessed on days 4-8 with the introduction of a tone as a conditioning stimulus (CS) and a rising rung (obstacle) as the unconditioned stimulus (US). These perturbations were used to evaluate mouse ability to adapt motor behavior in real time. Repeated perturbations were used to assess associative motor learning throughout successive trials. With the inter-stimulus interval fixed at $285 \mathrm{~ms}$, mice that associated the tone with the obstacle learned to increase walking speed to avoid being hit. In this sense, they decreased their 'pre-perturbation step time' (step before US) and their 'post-perturbation step time' (step just after the US). The Erasmus ladder is particularly valuable for detailed phenotyping of deficits associated with cerebellar pathology ${ }^{17}$

Rotarod. The accelerating rotarod performance test is a standard rodent assay for motor-associated functions such as coordination and balance. A 4-d testing paradigm (three sessions per day) was performed as previously described ${ }^{23}$ with a continuously accelerating rod that forces animals to modulate their balance. For the first $2 \mathrm{~d}$, animals were placed on the rod, which accelerated from 4 to 40 r.p.m. in 180 s. The test was stopped when the animal fell off the rod, was unable to maintain coordinated movements or 180 s passed. Additionally, if animals displayed 'cartwheeling', or clutching the rod and circling without walking, the test was stopped. To increase the challenge, for days 3-4 the acceleration of the rod increased from 8 to 80 r.p.m. in 180 s. The latency to fall from the rod and terminal velocity were then recorded. Linear regression analysis on data from each individual mouse was used to estimate initial motor coordination (given by the intercept) and learning rate (given by the slope).

Spontaneous behavior/stereotypies. Assessment of repetitive behavior was done to test autism-like behavior. Mice were allowed to habituate to the testing room before placement in a new, empty cage with bedding materials. A video camera was set with a lateral view to record spontaneous behavior for $15 \mathrm{~min}$. Videos were manually scored for time spent engaging in behaviors, including digging, gnawing, grooming, rearing and jumping.

Marble burying. The anxiety and stereotypic and/or obsessive-compulsive-like behavior components of ASD-like behavior can be assessed in mice by their behavioral responses to new 'diggable' media ${ }^{72,73}$ in the marble burying test. This test was performed in a clean, large $(26 \times 16 \mathrm{~cm})$ cage filled with $5 \mathrm{~cm}$ of bedding and 12 glass marbles evenly spaced on the bedding surface. The animal was left to explore the cage for 30 min undisturbed. Marbles covered 2/3 or more with bedding were counted as buried.

Socialization test. The three-chamber test (Crawley's paradigm ${ }^{74}$ ) was used to assess social behavior as previously described ${ }^{28}$. A three-chamber Plexiglas box was used with small openings in each dividing wall to allow free access to each chamber. The center chamber was kept empty, wereas the flanking chambers were each equipped with an identical wire cup. After a 10-min habituation period to allow for exploration of the three-chamber equipment (session 1), an unfamiliar adult mouse of similar weight and coloration was placed within the wire cup in one of the side chambers and an 'object' within the wire cup in the opposite chamber. The dividers were raised to allow the test subject to move freely throughout all three chambers of the apparatus over a 10-min test session (session 2). The second session was analyzed for social preference given by the social preference index (SPI). SPI was calculated as follows: if time spent with the mouse (social) is S and the object (non-social) is NS, then SPI $=S /(S+N S)$.
Determination of the autism composite severity score. We used the same procedure as previously described in ref. ${ }^{33}$. The score calculation allows for the combination of individual discrete symptoms of the autistic syndrome, in a continuous manner, so that higher values reflect higher severity of autistic-like behavior. For each mouse, we recorded their spontaneous behaviors (grooming, digging, rearing, jumping and gnawing) and determined their SPI in the three-chamber test. The significant readouts (digging time, gnawing time and SPI) were then $\mathrm{z}$-standardized and averaged to establish the autism composite severity score.

Statistics and reproducibility. Mouse assignment to groups was not randomized, because it relies on their known genotype ( $\mathrm{C}$ versus plKO), but mice from at least three different litters were used in each group for all experiments. No litter-associated effect was seen. All data analyses were conducted blinded to group allocation. Statistical analysis was carried out with GraphPad Prism 7 software. Detection of EYFP in Cyp19-cre:R26R-EYFP placenta and brain sections, and in situ hybridizations in placenta sections, were performed in at least three animals. Cell and area quantification on cerebellar immunofluorescent-labelled sections was done on six sections per animal, homogeneously distributed throughout the cerebellar vermis. Myelinated axon g-ratios were measured from more than 400 axons on one electron microscopy scan per animal. For western blot analyses, at least two technical replicates were performed. When more than ten samples, and, thus, several membranes, needed to be compared at once, two common samples were resolved in the different electrophoresis gels and used as reference for quantification. The sample sizes are shown in the legends and chosen to meet or exceed sample sizes typically used in the field. Robust regression and outlier removal methodology with $Q$ value equal to 1 was used to determine outliers. Before conducting all analyses, variable distributions were evaluated for normality. If distribution was not normal, non-parametric methods were used. Differential gene expression across development in $\mathrm{C}$ and plKO mice was analyzed using one-way ANOVA with Dunnett's multiple comparisons test. Comparisons between two groups were analyzed using parametric test (unpaired $t$-test with Welch's correction) or non-parametric test (Mann-Whitney test). The Holm-Sidak method was used for multiple unpaired $t$-test comparison. Analyses involving data from three or more groups while considering only one independent variable were performed using one-way ANOVA with Tukey's multiple comparisons test. Two-way ANOVA was used to compare means across two or more dependent variables. Two-way repeated-measures ANOVA with Sidak's multiple comparisons tests were used for the Erasmus ladder and the rotarod. Summary data are presented in the text as mean \pm s.e.m. from $n$ animals. Differences were considered significant at $P<0.05$. A supplementary statistics methods checklist is available.

Reporting Summary. Further information on research design is available in the Nature Research Reporting Summary linked to this article.

\section{Data availability}

Raw and processed data are available from the corresponding authors upon reasonable request. The RNA-seq data discussed in this publication have been deposited in NCBI's Gene Expression Omnibus (Vacher et al., 2021) and are accessible through GEO Series accession number GSE173440. Source data are provided with this paper.

\section{References}

51. Irwin, R. W. \& Brinton, R. D. Allopregnanolone as regenerative therapeutic for Alzheimer's disease: translational development and clinical promise. Prog. Neurobiol. 113, 40-55 (2014).

52. Ngo, D. H. \& Vo, T. S. An updated review on pharmaceutical properties of gamma-aminobutyric acid. Molecules 24, 2678 (2019).

53. Zhu, X. et al. A role of endogenous progesterone in stroke cerebroprotection revealed by the neural-specific deletion of its intracellular receptors. $J$. Neurosci. 37, 10998-11020 (2017).

54. Liere, P. et al. Novel lipoidal derivatives of pregnenolone and dehydroepiandrosterone and absence of their sulfated counterparts in rodent brain. J. Lipid Res. 45, 2287-2302 (2004).

55. Kechin, A., Boyarskikh, U., Kel, A. \& Filipenko, M. cutPrimers: a new tool for accurate cutting of primers from reads of targeted next generation sequencing. J. Comput Biol. 24, 1138-1143 (2017).

56. Goldstein, L. D. et al. Prediction and quantification of splice events from RNA-seq data. PLoS ONE 11, e0156132 (2016).

57. Anders, S., Pyl, P. T. \& Huber, W. HTSeq-a Python framework to work with high-throughput sequencing data. Bioinformatics 31, 166-169 (2015).

58. Sun, J., Nishiyama, T., Shimizu, K. \& Kadota, K. TCC: an R package for comparing tag count data with robust normalization strategies. BMC Bioinformatics 14, 219 (2013).

59. Kramer, A., Green, J., Pollard, J. Jr. \& Tugendreich, S. Causal analysis approaches in ingenuity pathway analysis. Bioinformatics 30, 523-530 (2014).

60. Livak, K. J. \& Schmittgen, T. D. Analysis of relative gene expression data using real-time quantitative PCR and the $2^{-\Delta \Delta C_{T}}$ method. Methods 25, 402-408 (2001). 
61. Cahill, L. S. et al. Preparation of fixed mouse brains for MRI. Neuroimage 60, 933-939 (2012).

62. de Guzman, A. E., Wong, M. D., Gleave, J. A. \& Nieman, B. J. Variations in post-perfusion immersion fixation and storage alter MRI measurements of mouse brain morphometry. Neuroimage 142, 687-695 (2016).

63. Lerch, J. P., Sled, J. G. \& Henkelman, R. M. MRI phenotyping of genetically altered mice. Methods Mol. Biol. 711, 349-361 (2011).

64. Nieman, B. J., Flenniken, A. M., Adamson, S. L., Henkelman, R. M. \& Sled, J. G. Anatomical phenotyping in the brain and skull of a mutant mouse by magnetic resonance imaging and computed tomography. Physiol. Genomics 24, 154-162 (2006).

65. Jones, D. K., Horsfield, M. A. \& Simmons, A. Optimal strategies for measuring diffusion in anisotropic systems by magnetic resonance imaging. Magn. Reson. Med. 42, 515-525 (1999).

66. Collins, D. L., Neelin, P., Peters, T. M. \& Evans, A. C. Automatic 3D intersubject registration of MR volumetric data in standardized Talairach space. J. Comput. Assist. Tomogr. 18, 192-205 (1994).

67. Avants, B. B. et al. A reproducible evaluation of ANTs similarity metric performance in brain image registration. Neuroimage 54, 2033-2044 (2011).

68. Dorr, A. E., Lerch, J. P., Spring, S., Kabani, N. \& Henkelman, R. M. High resolution three-dimensional brain atlas using an average magnetic resonance image of 40 adult C57Bl/6J mice. Neuroimage 42, 60-69 (2008).

69. Richards, K. et al. Segmentation of the mouse hippocampal formation in magnetic resonance images. Neuroimage 58, 732-740 (2011).

70. Steadman, P. E. et al. Genetic effects on cerebellar structure across mouse models of autism using a magnetic resonance imaging atlas. Autism Res. 7, 124-137 (2014).

71. Ullmann, J. F., Watson, C., Janke, A. L., Kurniawan, N. D. \& Reutens, D. C. A segmentation protocol and MRI atlas of the C57BL/6J mouse neocortex. Neuroimage 78, 196-203 (2013).

72. Egashira, N. et al. Impaired social interaction and reduced anxiety-related behavior in vasopressin Vla receptor knockout mice. Behav. Brain Res. 178, 123-127 (2007).

73. Gyertyan, I. Analysis of the marble burying response: marbles serve to measure digging rather than evoke burying. Behav. Pharmacol. 6, 24-31 (1995)

74. Nadler, J. J. et al. Automated apparatus for quantitation of social approach behaviors in mice. Genes Brain Behav. 3, 303-314 (2004).

\section{Acknowledgements}

We thank G. Leone for providing us with Cyp19a-Cre mice. We also would like to express our gratitude to J. Scafidi, L.-J. Chew and I. Papazoglou for their comments on the manuscript. We thank M. Yang, the head of the Neurobehavioral Core at Columbia University Medical Center, for giving us access to the equipment. We thank E. Rafikian for scoring the ultrasonic vocalizations. We also thank the contribution of families to the NIH NeuroBioBank, as well as J. Cottrell (NIH NeuroBioBank), who assisted us throughout the process of human tissue selection. We gratefully acknowledge the Children's Research Animal Facility, directed by J. Bradford, for taking care of our mouse colonies and the DC-IDDRC Animal Neurobehavioral Core, headed by J. Corbin, for providing advice, space and equipment to conduct the behavioral phenotyping of our mice. Light and confocal microscopic analyses were carried out at the DC-IDDRC's Cell and Tissue Microscopy Core. The DC-IDDRC cores are supported by National Institutes of Health NICHD U54HD090257 (V.G.). This work was funded by National Institutes of Health grants R01HD092593 and 3R01HD092593-S1 (A.A.P.), R37NS109478 (V.G.) and F31HD098886 (J.J.O.), the Simons Foundation (SFARI Pilot Award no. 572832; A.A.P.), the Children's National Board of Visitors (A.A.P. and V.G.) and the Research Foundation of Cerebral Palsy Alliance (no. 3720; A.A.P.).

\section{Author contributions}

C.-M.V. and A.A.P. conceived the project and designed the experiments. C.-M.V. and J.S performed the pilot experiments. J.J.O., H.L. and D.B. performed the genetic validation of the model. Y.I., K.H.-T., T.S. and C.-M.V. performed the RNA sequencing analysis. H.L. and S.S. performed the qRT-PCRs. C.-M.V. and J.S. performed the histological experiments. P.K. counted Purkinje cells. C.-M.V., J.S. and S.S. performed the behavioral experiments. A.S. and V.G. contributed to the Erasmus ladder data acquisition and analysis. C.C.-P., A.P. and C.-M.V. performed the electron microscopy experiments. S.S. and H.L. performed the molecular and biochemical experiments on human tissues. P.L. and M.S. performed the mass spectrometry experiments. J.E. and J.P.L. performed the brain MRI. S.S. and J.S. performed the genotyping. C.-M.V. and A.A.P. wrote the manuscript. All authors reviewed and revised the manuscript.

\section{Competing interests}

The authors declare no competing financial interests.

\section{Additional information}

Extended data is available for this paper at https://doi.org/10.1038/s41593-021-00896-4. Supplementary information The online version contains supplementary material available at https://doi.org/10.1038/s41593-021-00896-4.

Correspondence and requests for materials should be addressed to C.-M.V. or A.A.P.

Peer review information Nature Neuroscience thanks Margaret McCarthy and the other, anonymous, reviewer(s) for their contribution to the peer review of this work.

Reprints and permissions information is available at www.nature.com/reprints. 
a

ALLO levels

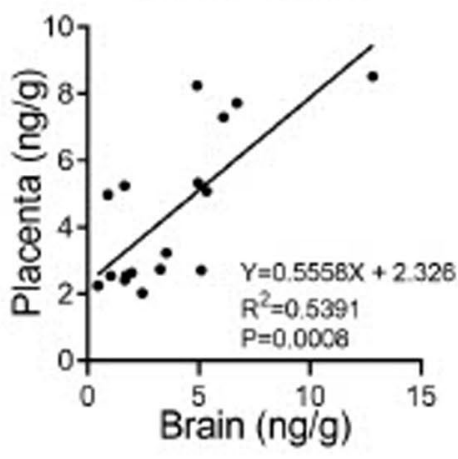

d
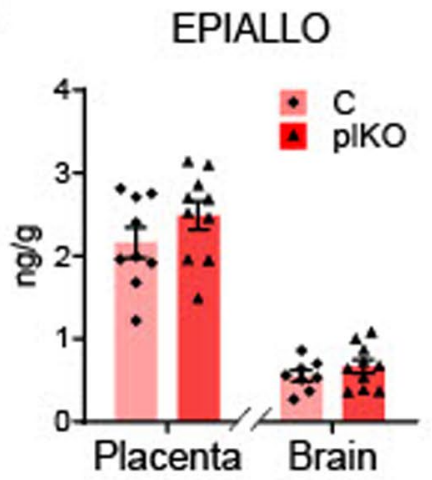

b

PREG

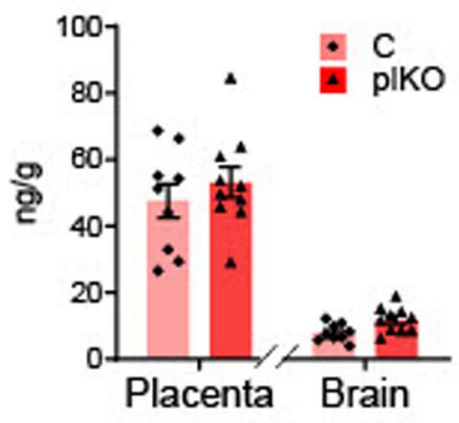

e

ALLOTHDOC

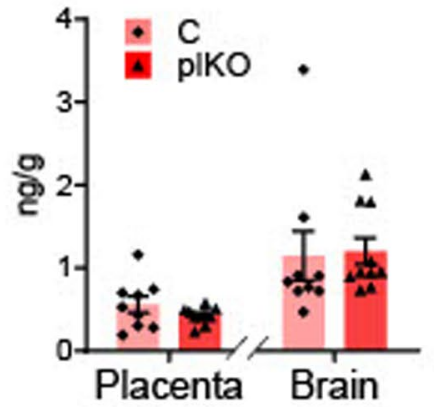

C

PROG

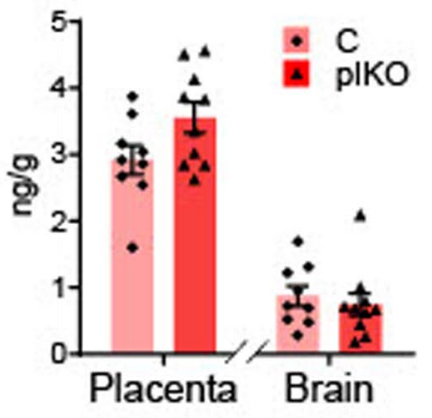

f

Androstanediol

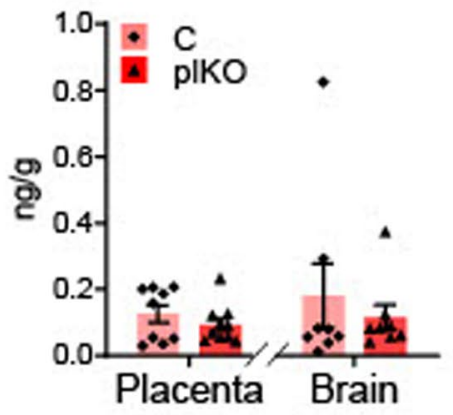

Extended Data Fig. 1 | Chemical validation of the pIKO model by mass spectrometry. a, Linear regression of ALLO levels in placenta and brain, all sexes and genotypes included. ALLO levels in placenta and brain show a positive correlation $(p=0.0008 ; n=18)$. $\mathbf{b}-\mathbf{f}$, Mass spectrometry data in placenta and brain at E17.5. Data is presented as means $\pm \operatorname{SEM}(n=9 \mathrm{C}$ and $10 \mathrm{pIKO})$. Two-way ANOVA with Sidak's multiple comparison test. Sex mixed as no sex effect was evidenced. ALLO, allopregnanolone; EPIALLO, epiallopregnanolone; PREG, pregnenolone; PROG, progesterone; ALLOTHDOC, allotetrahydrodeoxycorticosterone. 
$\begin{array}{llllll}\text { a } & \text { efnb3 } & \text { b } & \text { sbf1 } & \text { C } & \text { fabp7 }\end{array}$
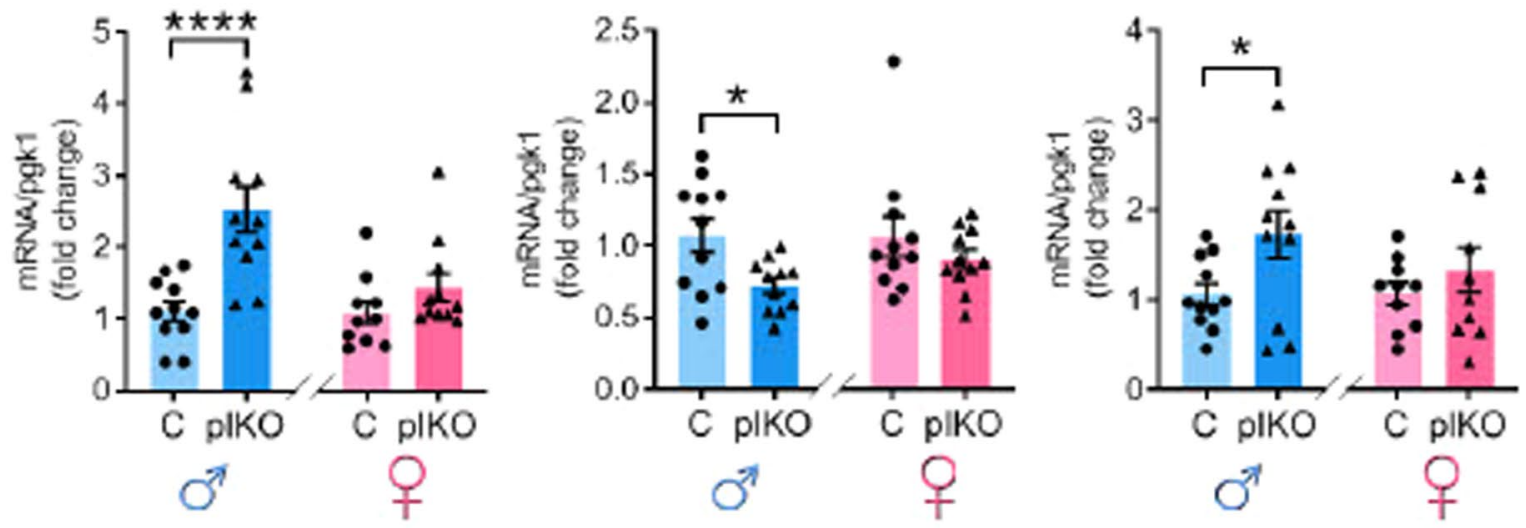

d tcf7/2

e nf1

f pip1
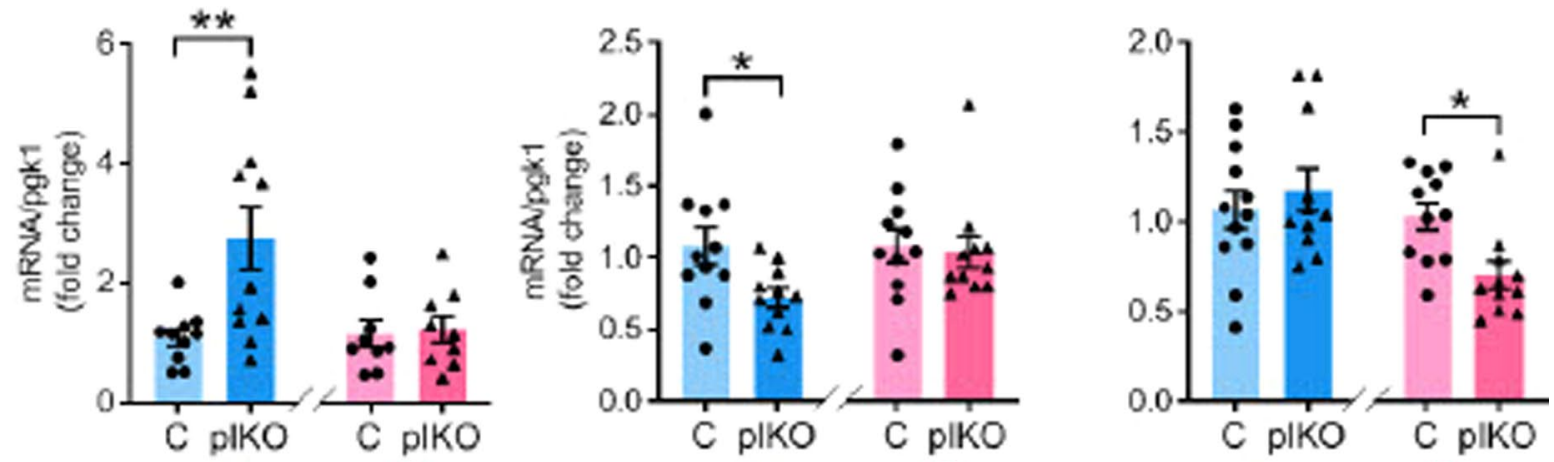

g
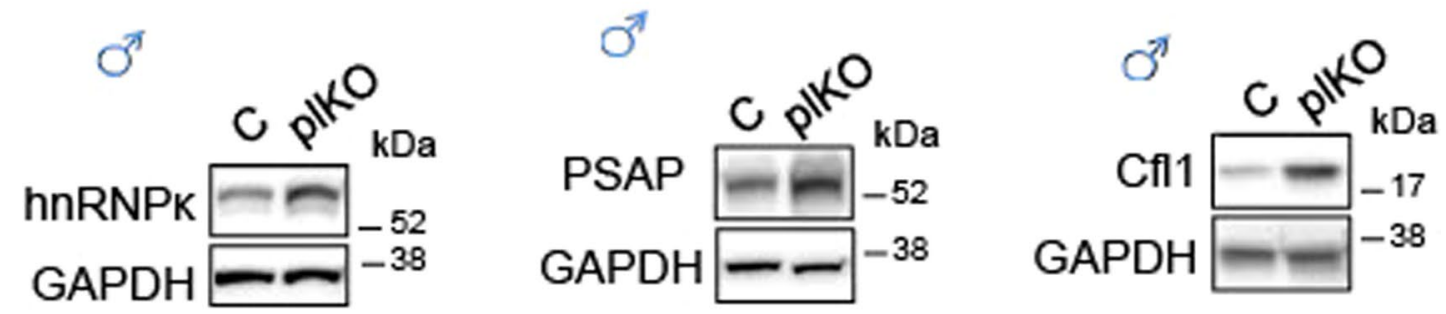

h
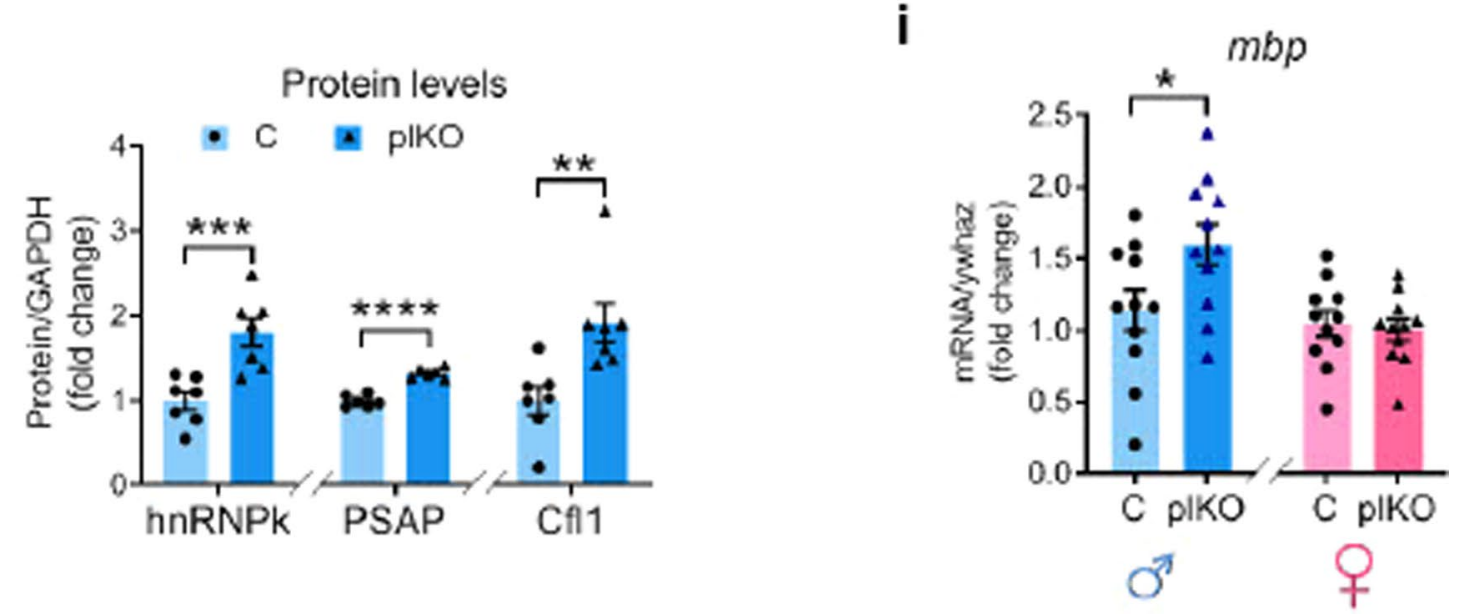

Extended Data Fig. 2 | See next page for caption. 
Extended Data Fig. 2 | Molecular validation of the RNA sequencing cutoffs. Changes in mRNA (a-f) or protein ( $g$-h) contents of selected cerebellar dysregulated genes after normalization. a, efnb3, ephrinB3 (RNAseq rank \#26/1192 in males; up-regulated; $q=0.018 ; p=3.41 E-05$ ). b, sbf1, SET Binding Factor 1 (RNAseq rank \#82/1192 in males; down-regulated; $q=0.067 ; p=0.00039$ ). $\mathbf{c}$, fabp7, Fatty Acid Binding Protein 7 (RNAseq rank \#84/1192 in males; up-regulated; $q=0.0725 ; p=0.00043$ ). $\mathbf{d}$, $t c f 712$, transcription factor 7 like 2 (RNAseq rank \#795/1192; up-regulated; $q=0.514 ; p=0.029$ ). e, $n f 1$, Neurofibromin 1 (RNAseq rank \#1189/1192 in males; down-regulated; $q=0.59 ; p=0.0499$ ). f, plp1, Proteolipid Protein 1 (RNAseq rank \#64/389 in females; down-regulated; $q=1 ; p=0.0054$ ). Normalized $q R T-P C R$ data (to $p g k 1$ ) is presented as mean fold changes $\pm S E M, n=10$ samples/group.). Twoway ANOVA with Sidak's multiple comparison test (a-f). ${ }^{\star} p<0.05,{ }^{\star \star} p<0.01,{ }^{\star \star \star \star} p<0.0001$. g-h, Cerebellum protein contents determined by Western blot in males, and normalized to GAPDH levels. $\mathrm{hnRNP}_{\mathrm{K}} / \mathrm{Cf11}: \mathrm{n}=7 \mathrm{C}$ and $7 \mathrm{pIKO} ; \mathrm{PSAP}: \mathrm{n}=6 \mathrm{C}$ and 6 plKO. Multiple unpaired $\mathrm{t}$ tests with Holm-Sidak multiple comparison test $\left({ }^{\star \star} p<0.01,{ }^{\star \star \star} p<0.005,{ }^{\star \star \star \star} p<0.0001\right)$. PSAP, prosaposin (RNAseq rank \#950/1192 in males; up-regulated; $q=0.5329$; $p=0.0359$ ). Cfl1, cofilin-1 (RNAseq rank \#27/1192 in males; up-regulated; $q=0.0192 ; p=4.03 E-05$ ). hnRNPк, heterogeneous nuclear ribonucleoprotein $\kappa$ (rank \#787; up-regulated; $q=0.509 ; p=0.0284$ ). i, mbp, myelin basic protein. Normalized qRT-PCR data (to ywhaz, Tyrosine 3-Monooxygenase/ Tryptophan 5-Monooxygenase Activation Protein Zeta). Two-way ANOVA with Sidak's multiple comparison test $\left(n=11 \mathrm{C}\right.$ and $11 \mathrm{plKO}$; $\left.{ }^{\star} \mathrm{p}<0.05\right)$. See Extended Data Table 1 sheets 1 and 2 for list of ranked DEGs. 

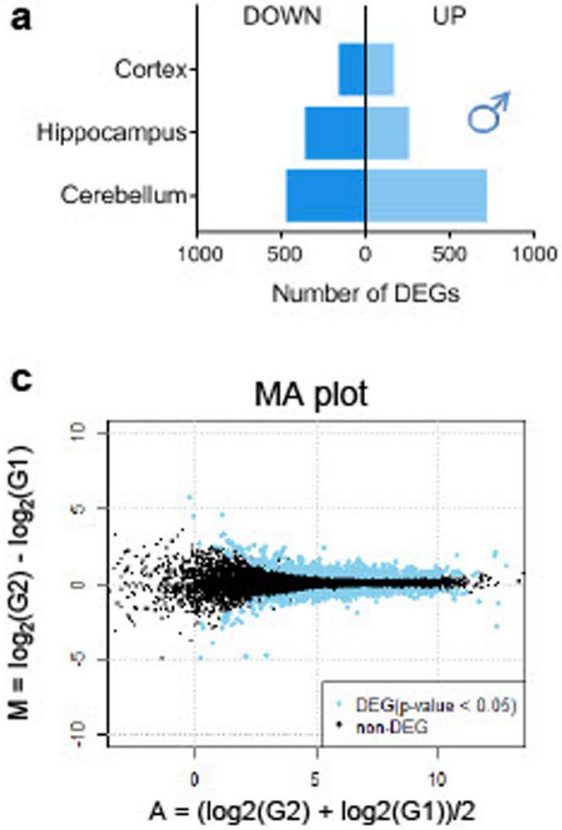

e

\begin{tabular}{|cc|}
\hline Diseases and Functions Annotation in Males & p value \\
\hline atrophy of white matter & $5.34 \mathrm{E}-03$ \\
abnormal morphology of white matter & $9.47 \mathrm{E}-03$ \\
long-term potentiation & $1.69 \mathrm{E}-02$ \\
migration of Purkinje cells & $1.76 \mathrm{E}-02$ \\
cell-cell contact & $2.62 \mathrm{E}-02$ \\
apoptosis & $3.15 \mathrm{E}-02$ \\
\hline
\end{tabular}

g

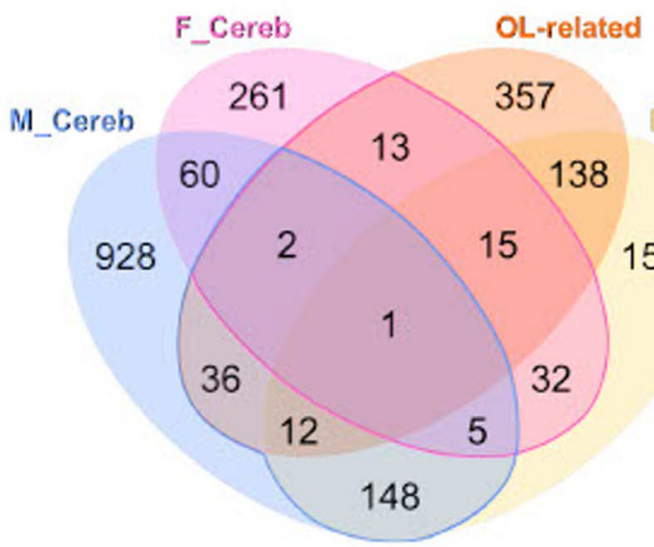

M_Cereb $\cap(\mathrm{OL} \cup$ Myelin $)=\mathbf{2 0 4}$ genes

F_Cereb $\cap(\mathrm{OL} \cup$ Myelin $)=68$ genes

f
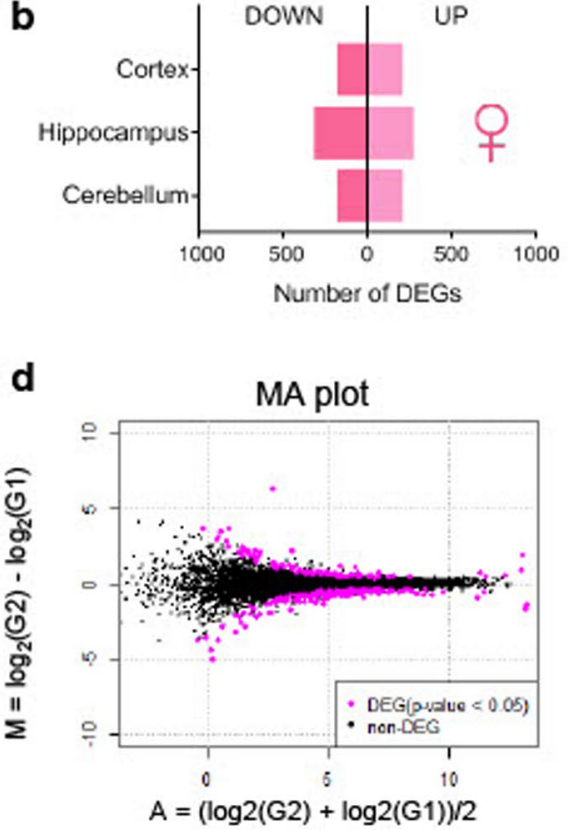

\begin{tabular}{|cc|}
\hline Diseases or Functions Annotation in Females & P value \\
\hline microtubule dynamics & $1.23 \mathrm{E}-02$ \\
cell death of granule cells & $1.81 \mathrm{E}-02$ \\
synaptic transmission & $1.94 \mathrm{E}-02$ \\
neurotransmission & $2.82 \mathrm{E}-02$ \\
abnormal morphology of white matter & $4.18 \mathrm{E}-02$ \\
apoptosis & $4.44 \mathrm{E}-02$ \\
\hline
\end{tabular}

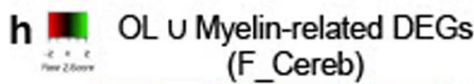

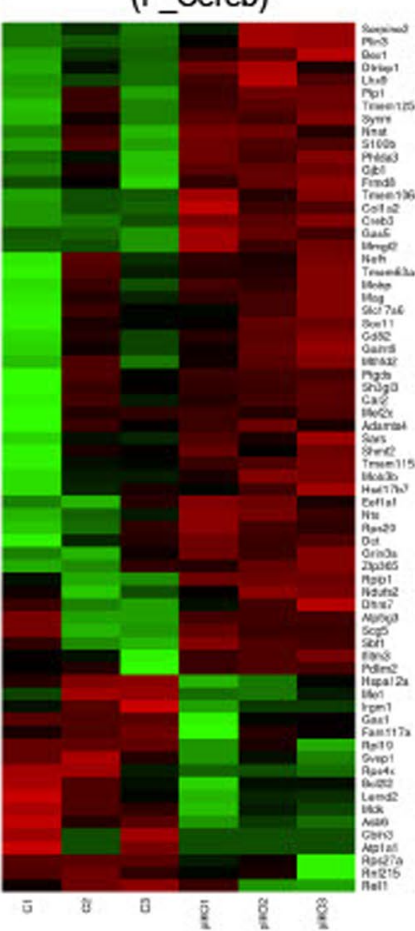

OL U Myelin-related DEGs (M_Cereb)

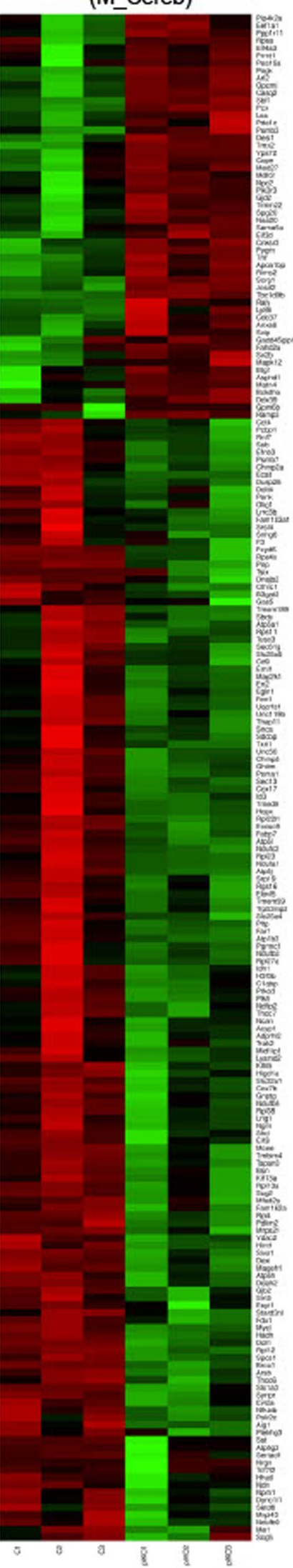


Extended Data Fig. 3 | Long-term impact of placental ALLO insufficiency on brain transcriptome: highlight on the myelin-associated genes in the cerebellum. $\mathbf{a}-\mathbf{b}$, Number of differentially expressed genes (DEGs) that are up- or down-regulated in the cerebral cortex, hippocampus and cerebellum of pIKO vs $C$ mice at P3O in males (blue) and females (pink). The majority of DEGs were found in the male cerebellum. c-d, MA plots for the cerebellar RNAseq analysis of pIKO vs C mice at P30. M, log ratio; A, mean average. DEGs are represented as colored dots (blue for males and pink for females). e-f, Top significant diseases and functions IPA annotations. g, Venn diagrams showing the overlaps between cerebellar DEGs in the pIKO males (M_Cereb) and females (F_Cereb), and oligodendrocyte (OL) and myelin transcriptomes ${ }^{3,4} \cdot \mathbf{h}-\mathbf{i}$, Heatmaps of OL-and myelin-related cerebellar DEGs in C and pIKO samples. Genes and samples were hierarchically clustered based on Pearson distance of $z$-score data and average linkage. $n=3$ independent samples/ group 7.8 . 


\section{Mean FA in cerebellar tracts}

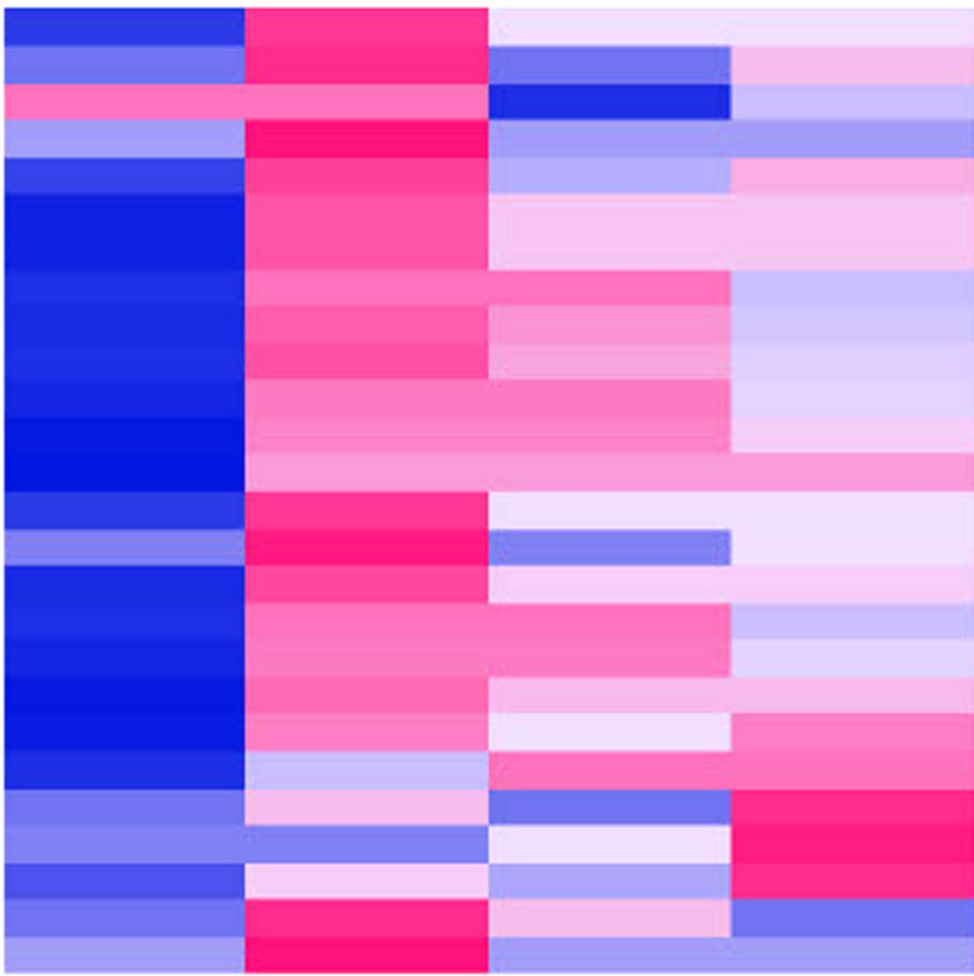

cerebellum related fiber tracts cerebellar peduncles

inferior cerebellar peduncle middle cerebellar peduncle superior cerebellar peduncle arbor vitae

trunk of arbor vita

lobule 1-2 white matter

lobule 3 white matter

trunk of lobules 1-3 white matter

lobules 4-5 white matter lobules 6-7 white matter lobule 8 white matter trunk of lobules $6-8$ white matter lobule 9 white matter lobule 10 white matter anterior lobule white matter simple lobule white matter crus 1 white matter trunk of simple and crus 1 white matter crus 2 white matter paramedian lobule trunk of crus 2 and paramedian white matter copula white matter paraflocculus white matter flocculus white matter

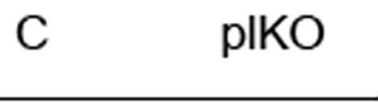

males

\section{C $\quad$ plKO}

L

\section{females}

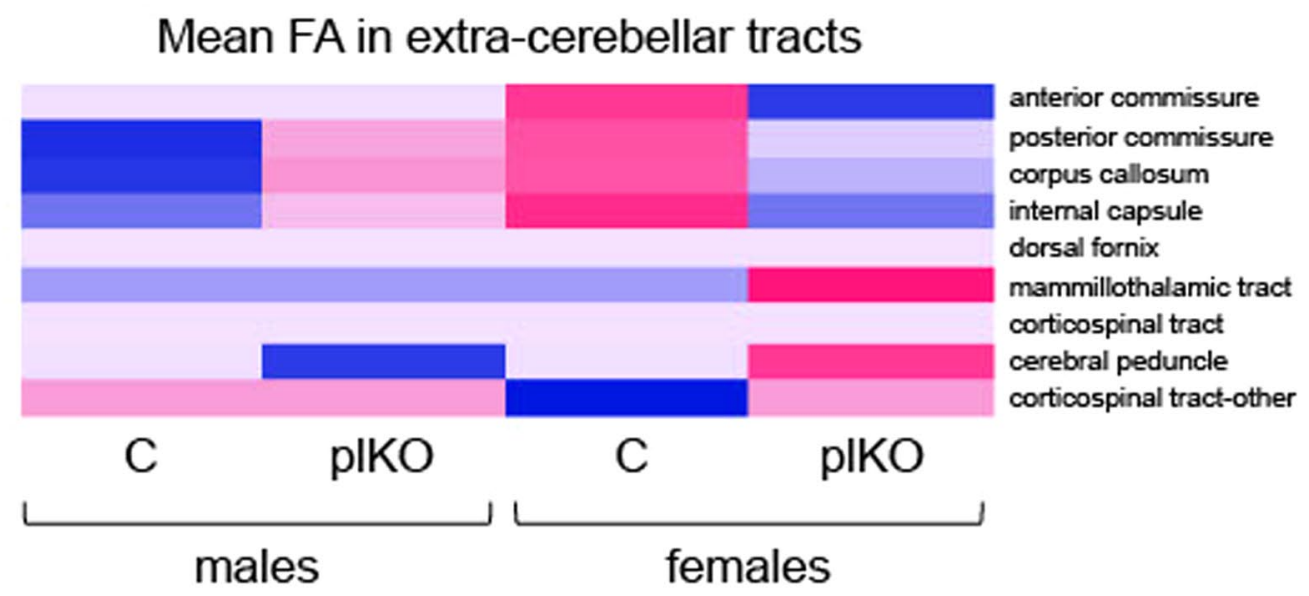

Row Z-score

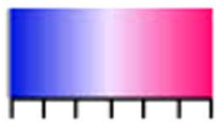


Extended Data Fig. 4 | Diffusion Tensor Image (DTI) analysis at P30. a, Heatmap representing mean fractional anisotropies (FA) in the cerebellum related tracts. Mean FA is increased in the cerebellar WM of pIKO males compared to $C$ littermates. Data is presented in a hierarchical tree [region effect: $F(25,1014)=57.36, p<0.0001$; sex effect: $F(1,1014)=2.097, p=0.1479$; genotype effect: $F(1,1014)=16.99$, $p<0.0001$; sex $\times$ genotype interaction: $F(1,1014)=17.92, p<0.0001 ; 3$-way ANOVA]. b. Heatmap representing mean FA in extra-cerebellar tracts. No significant change was evidenced between groups when compared for genotype or sex [region effect: $F(8,351)=80.28, p<0.0001$; sex effect: $F(1,351)=1.011, p=0.3152$; genotype effect: $F(1,351)=0.1618, p=0.6877$; sex $\times$ genotype interaction: $F(1,351)=2.589, p=0.1021 ; 3$-way ANOVA]. Males: $n=11 \mathrm{C}$ and $13 p \mid K O ; F e m a l e s: n=8 C$ and 11 pIKO. 
a

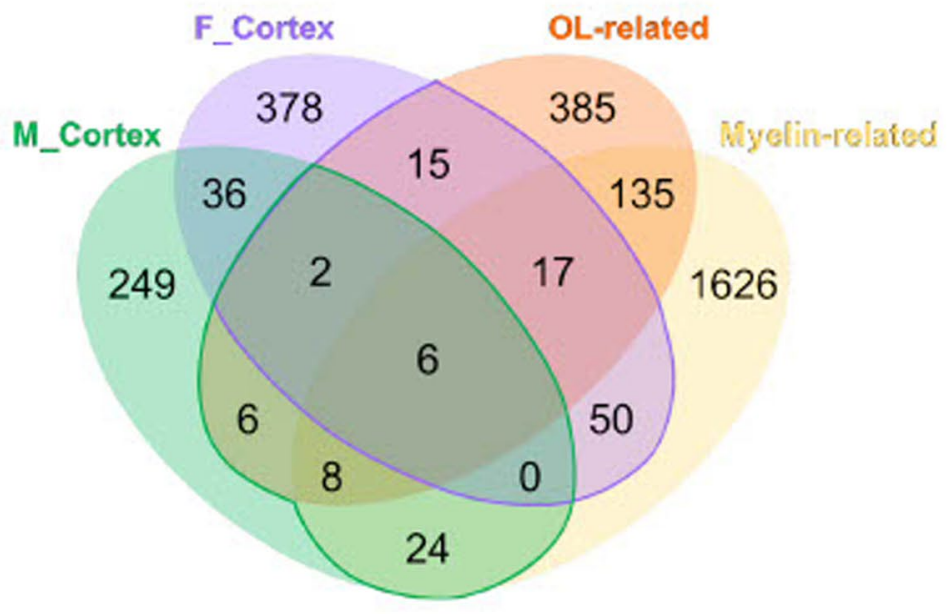

M_Cortex $\cap(\mathrm{OL} \cup$ Myelin $)=46$ genes

F_Cortex $\cap(\mathrm{OL} \cup$ Myelin $)=90$ genes

b

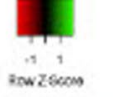

OL/Myelin-related DEGs (M_Cortex)

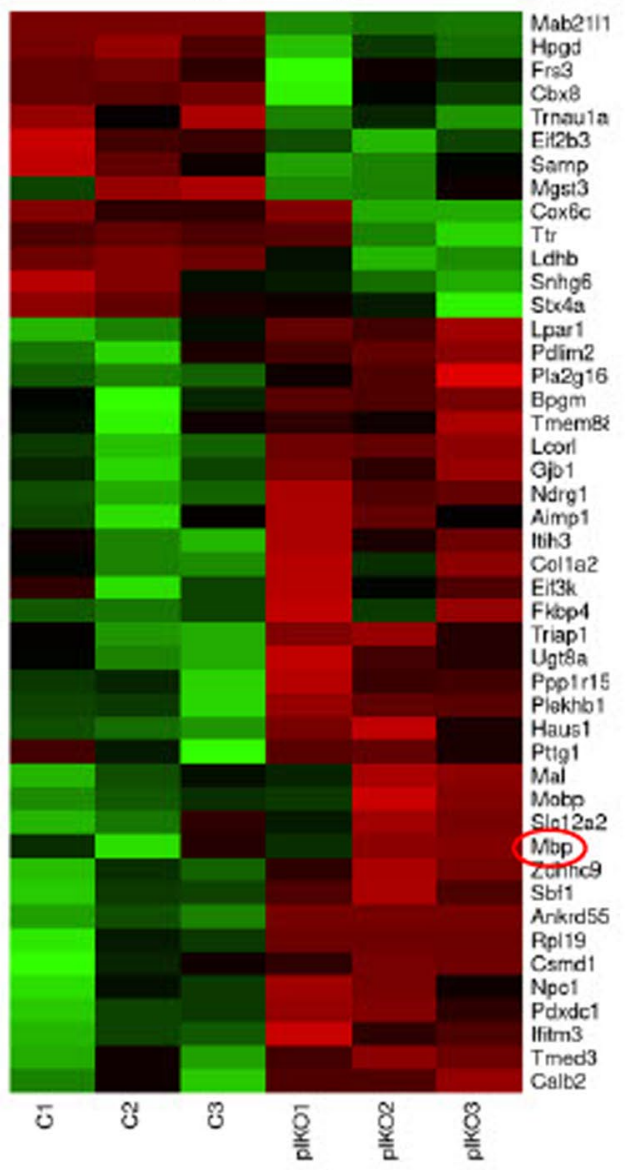

C
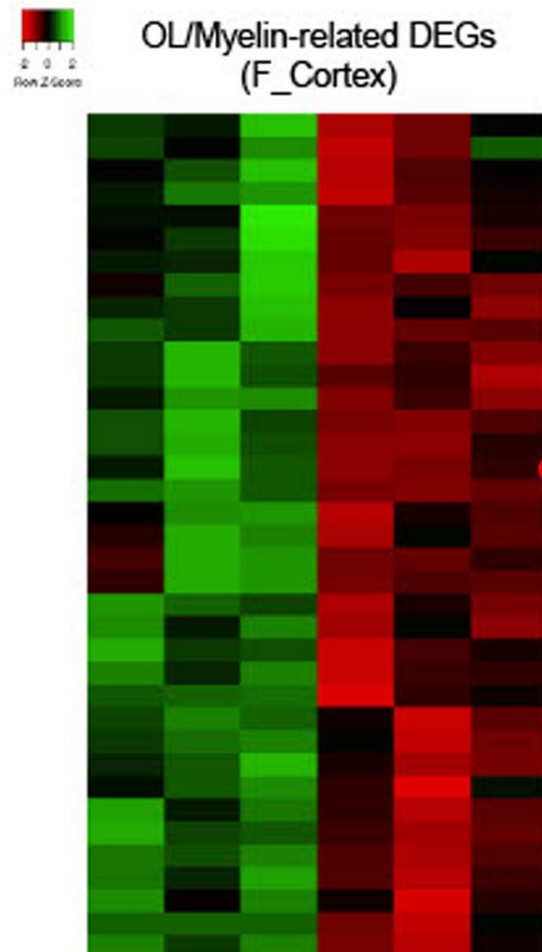

Kesd 13 Mrpl2 $\operatorname{sen} 14 a$ Apasiti: Mgst3

sotis

S100a1 $\mathrm{H} 3 \mathrm{Bb}$ Ewecs Tox264 Mpp coss Sors Ppp1r14 Nofufte6 Rplp1 Sinpe gsto impo Nis Fmod Nkmo.2 Gatn Snnpeli Socpch Pirg1 Sorti Math1

Gag

Tsem

Tsim

Quedc2 Nithto2 Ieraipt Mobp Cур.2 25 Sik11 Mag Gat10 Dat Timern11 Pencta Ndufs? Sag 5 sus Chepe par1 Fazh Aramise Jumis Dodah2 Lysmed2 To Ednt Tus:3 Soink8 Potim2 Atp5: Nounte Adant Jup Jinern4 1 Passine Npc1 Fepa 12 \& Frt2 Aiplat Big2 Fosp1 Sicla? Fos Jư⿱宀 Avc R3hom 1 ler2 Egr? Dusp! Te.k1 Tuso3 Pmp22

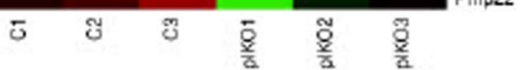

Extended Data Fig. 5 | See next page for caption. 
Extended Data Fig. 5 | Long-term impact of placental ALLO insufficiency on cerebral cortex transcriptome at P30: highlight on the OL- and myelin-related genes. a, Venn diagrams showing the overlaps between cortical DEGs in the pIKO males (M_Cortex) and females (F_Cortex), oligodendrocyte (OL) and myelin transcriptomes ${ }^{3,4}$. b-c, Heatmaps of OL-and myelin-related DEGs in the cerebral cortex of $\mathrm{C}$ and pIKO samples. Genes and samples were hierarchically clustered based on Pearson distance of $z$-score data and average linkage. $n=3$ independent samples/group. 


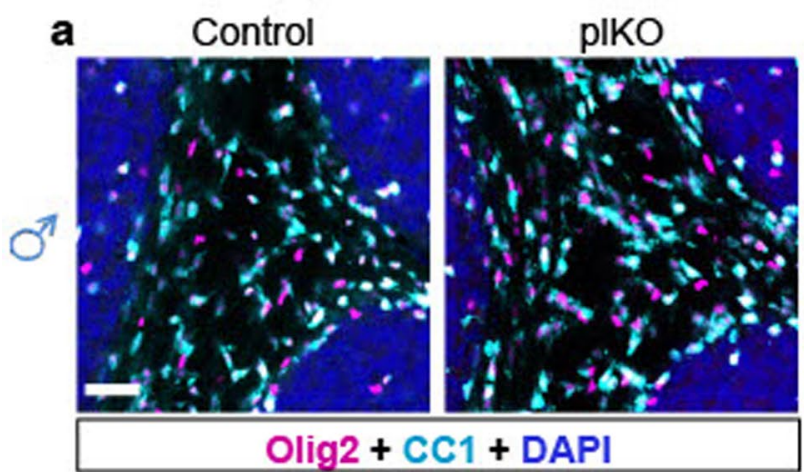

b

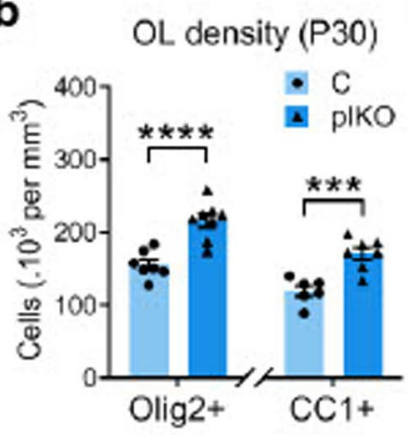

C $\mathrm{BrdU}+(\mathrm{E} 15.5)$ /Olig2 $+(\mathrm{P} 30)$

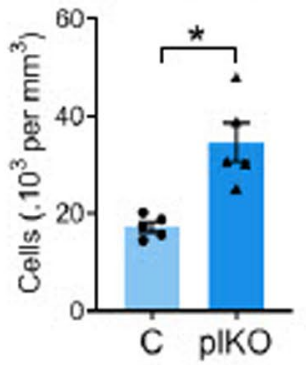

d

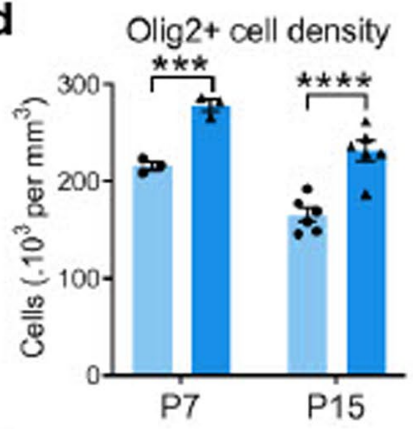

g

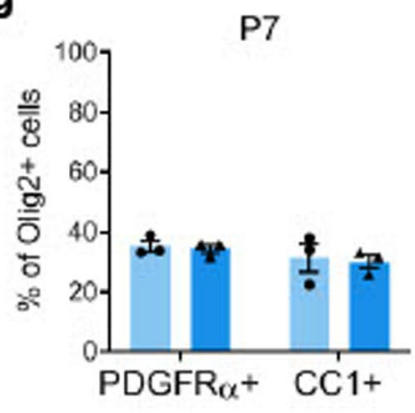

j

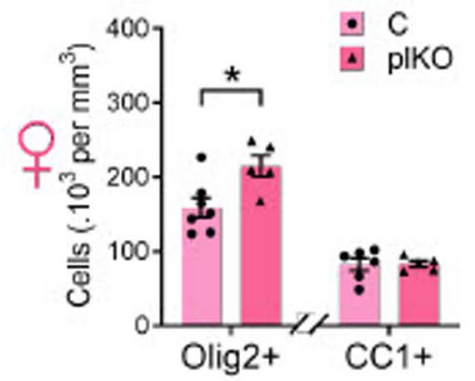

e

PDGFR $\alpha+$ cell density

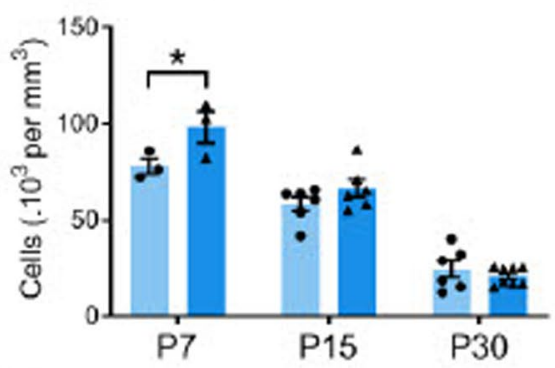

h

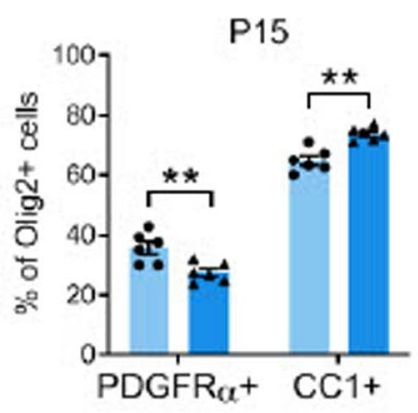

k

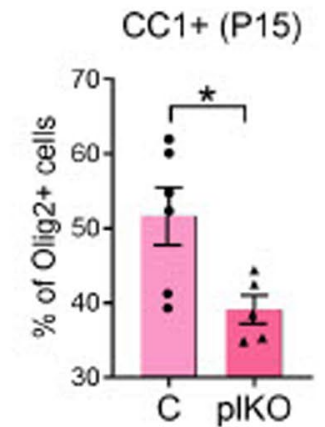

Extended Data Fig. 6 | See next page for caption.

f $\quad$ CC1+ cell density

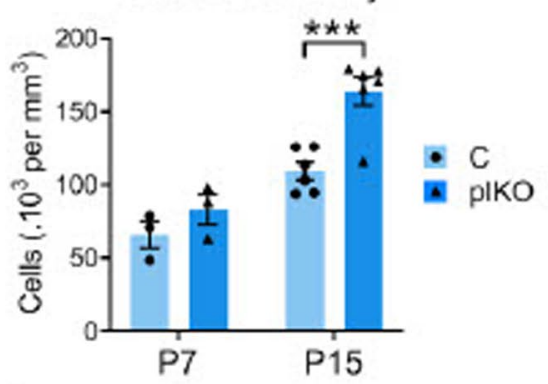

i

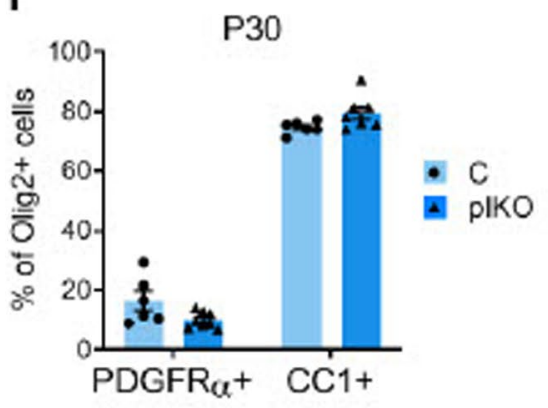


Extended Data Fig. 6 | Oligodendrocyte lineage progression during the postnatal period. a, Immunofluorescent staining of Olig2 and CC1 in inter-lobule VI-VII WM at P30. Scale bar, $60 \mu \mathrm{m}$. b, Quantification of Olig2+ and CC1+ cell density in the male cerebellar WM at P30. Data presented as means \pm SEM. Two-way ANOVA with Sidak's multiple comparisons test $\left({ }^{\star \star \star} p<0.005,{ }^{\star \star \star \star} p<0.0001\right)$. Olig2: $n=7 \mathrm{C}$ and 8 pIKO $(p<0.0001) ; C C 1: n=6 C$ and 7pIKO ( $p=0.0005)$. c, Quantification of BrdU + /Olig2+ cell density in the male cerebellar WM at P30. BrdU (50 mg/kg) was injected to dams at E15.5. Data presented as means \pm SEM. Two-tailed unpaired Student's $t$ test with Welch's correction $\left({ }^{*} p<0.05\right) . n=5 C$ and $5 p \mid K O(p=0.0107)$. d, Quantification of Olig2+ cell density in the male cerebellar WM at P7 and P15. Data is presented as means \pm SEM. Two-way ANOVA with Sidak's multiple comparisons test $\left({ }^{\star \star \star} p<0.005,{ }^{\star \star \star \star} p<0.00010\right)$. P7: $n=3$ mice/group $(p=0.0026) ; P 15: n=6$ mice/group $(p<0.0001)$. The density of Olig2+ cells in the cerebellar WM is significantly increased in pIKO males as compared with C littermates. e, OPC (PDGFR $\alpha+$ cell) density at P7, P15 and P30. Data is presented as means \pm SEM. Two-way ANOVA with Sidak's multiple comparisons test ( $\left.{ }^{\star} p<0.05\right)$. P7: $n=3$ mice/group $(p=0.0435)$; P15: $n=6$ mice/ group ( $p=0.3644) ; P 30: n=6 C$ and 8 pIKO ( $p=0.8558)$. The density of OPCs in the cerebellar WM is transiently higher in plKO males as compared with C littermates at P7. f, Quantification of mature OL $(C C 1+$ cell) density within the male cerebellar WM at P7 and P15. Data is presented as means \pm SEM. Two-way ANOVA with Sidak's multiple comparisons test ( $\left.{ }^{\star \star \star} p<0.005\right)$. P7: $n=3$ mice/group $(p=0.4951) ; P 15: n=6$ mice/group ( $\left.p<0.0004\right)$. The density of mature OLs in the cerebellar WM is significantly increased in pIKO males as compared with C littermates at P15. g-i, Ratios of OPCs (PDGFR $\alpha+$ cells) or mature OLs (CC1+ cells) within the total oligodendrocyte lineage (Olig2 + cells) in the male cerebellar WM across postnatal period. Data is presented as means \pm SEM. Two-way ANOVA with Sidak's multiple comparisons test (** $p<0.005)$. P7: $n=3$ mice/group [p=0.9741 (PDGFR $\alpha$, $p=0.9393$ (CC1)]; P15: $n=6$ mice/group $[p=0.002$ (PDGFR $), p=0.001(C C 1)] ; P 30: n=7 C$ and $8 p \mid K O[p=0.052(P D G F R \alpha), p=0.17(C C 1)]$. At P15, a transient acceleration of the OL maturation is observed in the male plKO. $\mathbf{j}$, Quantification of total OL lineage $(\mathrm{Olig} 2+)$ and mature OL $(\mathrm{CC} 1+)$ cell densities in the female cerebellar WM at P15. Data is presented as means \pm SEM. Two-way ANOVA with Sidak's multiple comparisons test ( $\left.{ }^{*} p<0.01\right)$. Olig2 $+: n=7 C$ and 5 pIKO $(p=0.0058) ; C C 1+: n=6 C$ and 5 pIKO $(p=0.9984)$. The density of total OL is increased in the cerebellar WM of pIKO females compared to C littermates. k, Ratio of mature OLs (CC1+ cells) within the total OL lineage (Olig2 + cells) in the female cerebellar WM at P15. Data is presented as means \pm SEM. Two-tailed unpaired Student's t test with Welch's correction $\left.{ }^{*} p<0.05\right) . n=6 C$ and $5 p I K O(p=0.0225)$. A significantly reduction of $\mathrm{OL}$ maturation is observed in the female pIKO as compared with littermate $\mathrm{C}$. 

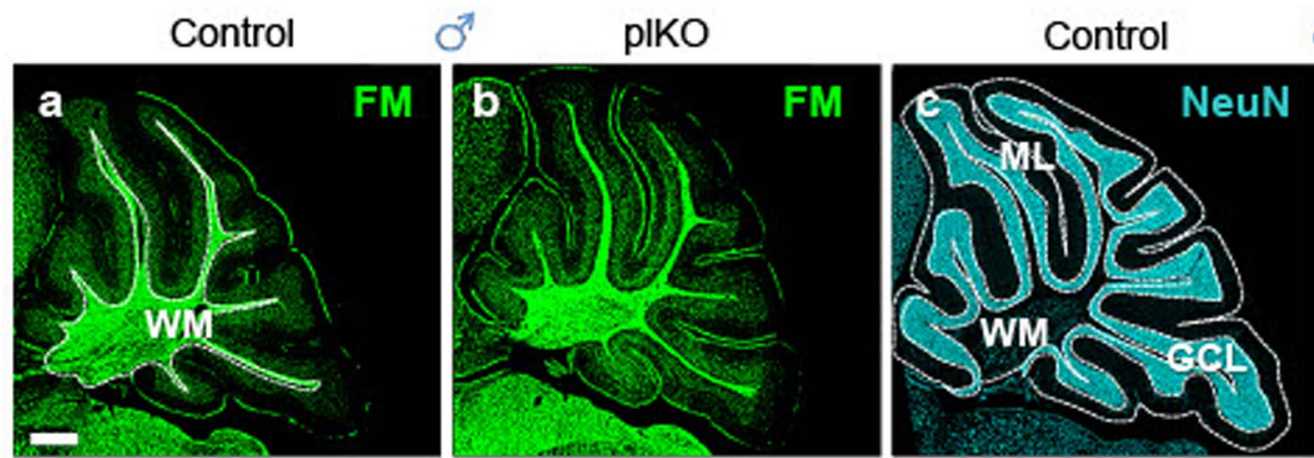

O $\quad$ PlKO
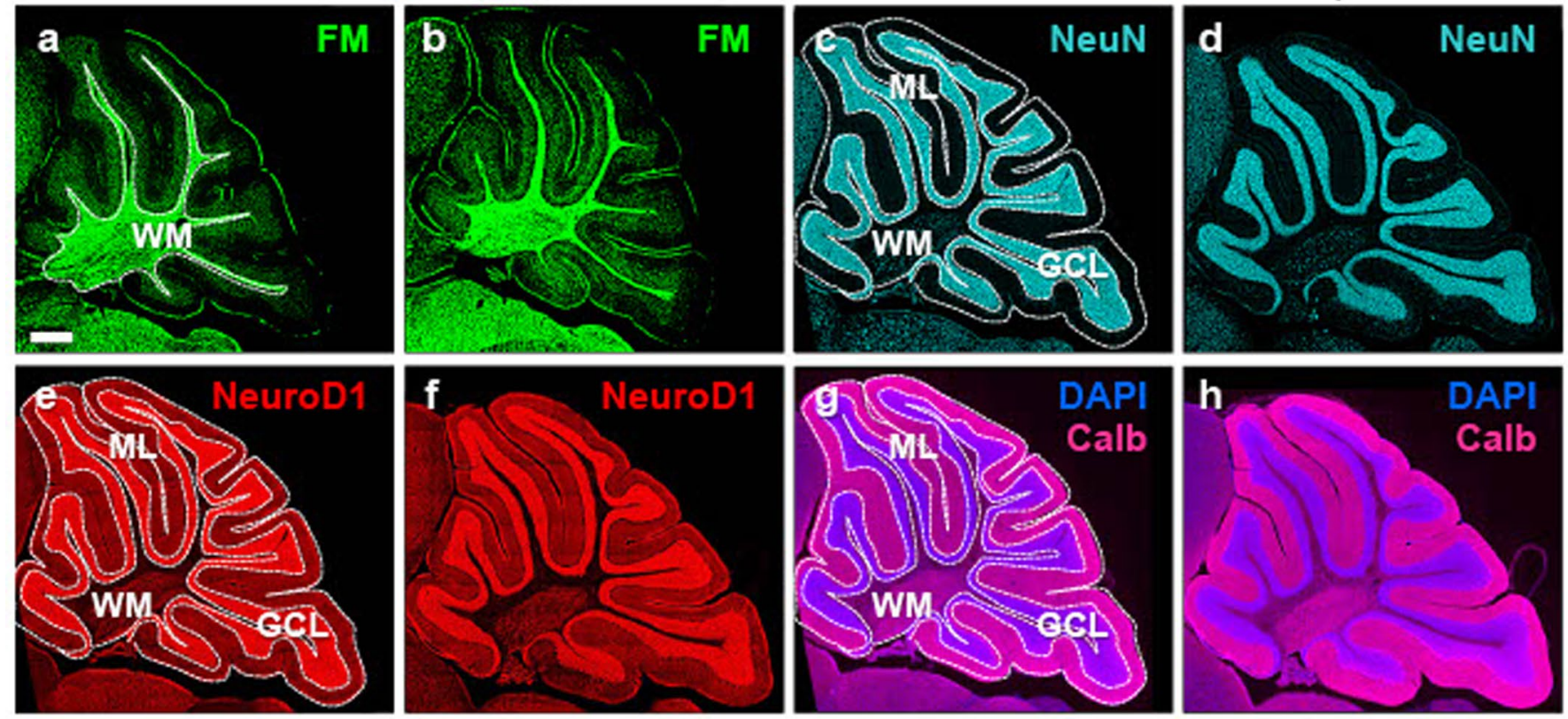

i

Relative cerebellar layer volumes

j
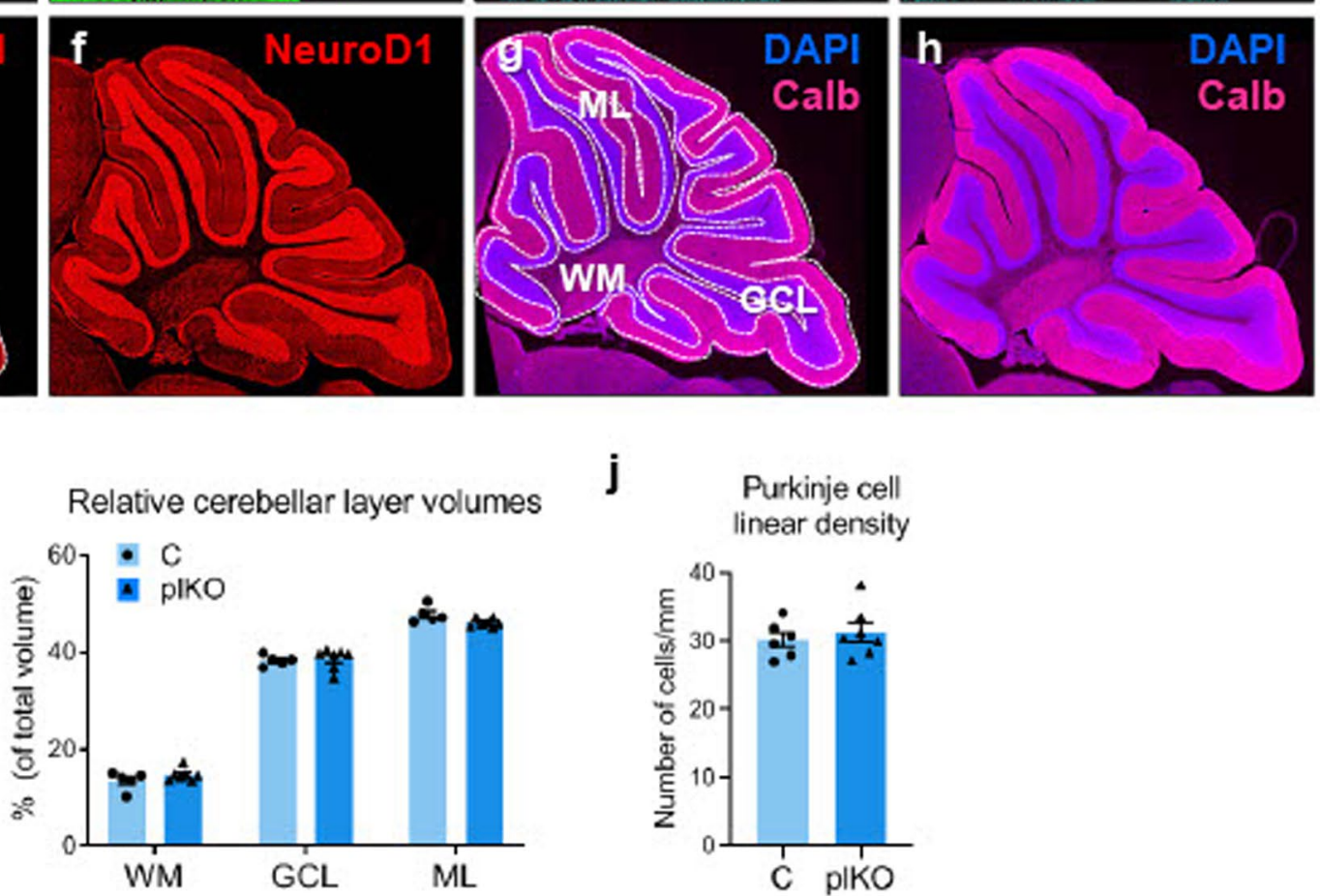

Purkinje cell linear density

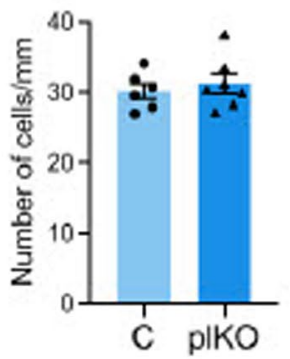

Extended Data Fig. 7 | Anatomical analysis of cerebellar grey and white matters in C and plKO at P30. a-h, Cerebellar sagittal 40- $\mu$ m-thick sections through the vermis in males. a-b, FluoroMyelin (FM) green staining. c-d, NeuN-immunofluorescence (marker for neuronal nuclei). e-f, NeuroD1-immunofluorescence (marker for granule cells). g-h, Calbindin-immunofluorescence (Purkinje cell marker; in magenta) and DAPI (in blue). $\mathbf{i}$, Cerebellar layer volumes were unchanged in pIKO when compared with C mice. Data is presented as means \pm SEM from 6 sections/animal. Two-way ANOVA with Sidak's multiple comparisons test. $n=5 \mathrm{C}$ and 7pIKO (WM: $p=0.48 ; G C L: p=0.98 ; M L: p=0.22$ ). $\mathbf{j}$, Purkinje cell linear density in the lobule VI-VII of $C$ and pIKO males at P30. Data is presented as means \pm SEM from 6 sections/animal. Two-tailed unpaired Student's $t$ test with Welch's correction. $n=6 C$ and 7pIKO ( $p=0.556$ ). Calb, calbindin; $G C L$, granule cell layer; $M L$, molecular layer; WM, white matter. Scale bar, $200 \mu m$. 
a

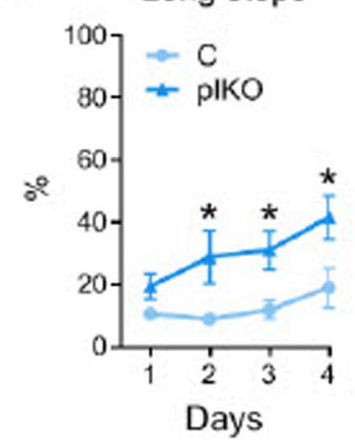

e

Pre-perturbation time

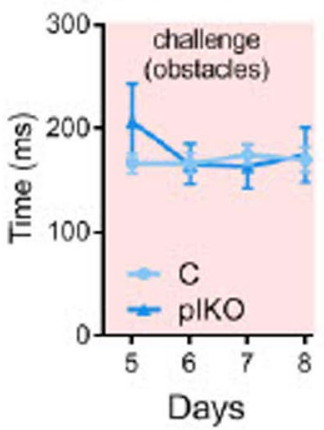

i

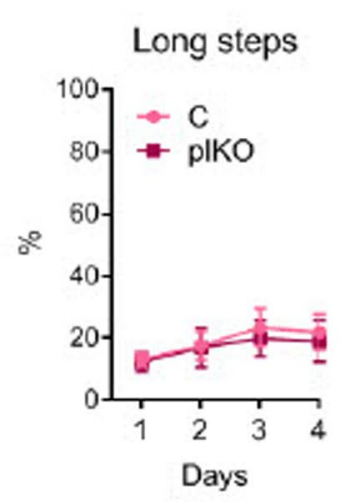

b

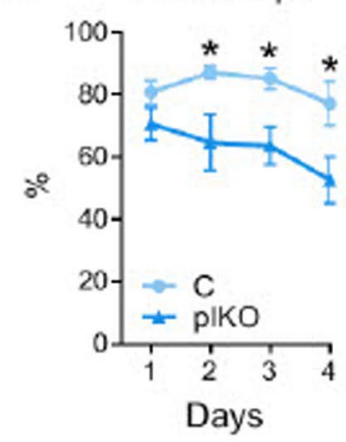

f Post-perturbation time

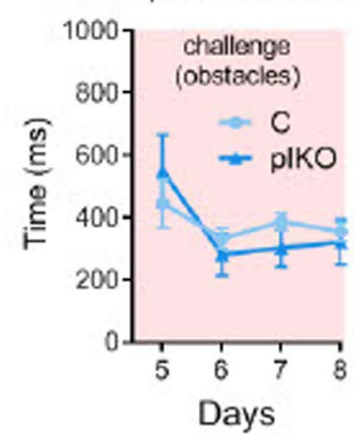

j

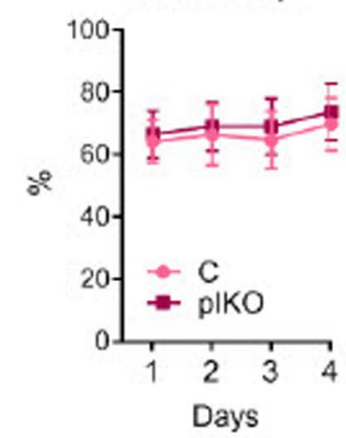

c

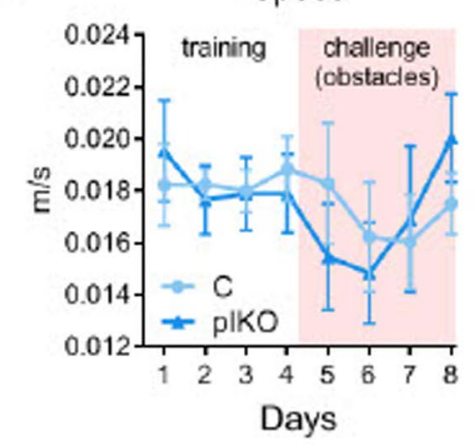

g Missteps

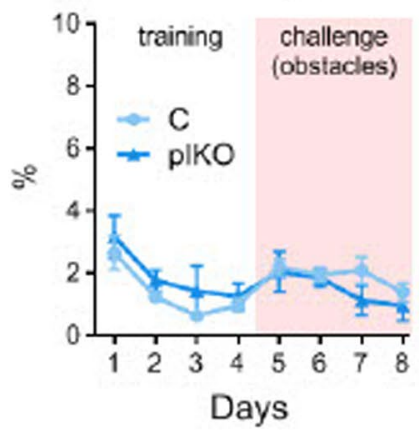

$\mathbf{k}$

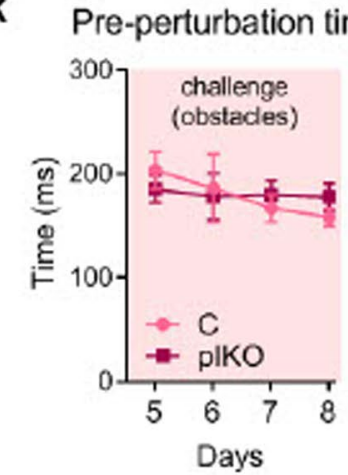

d

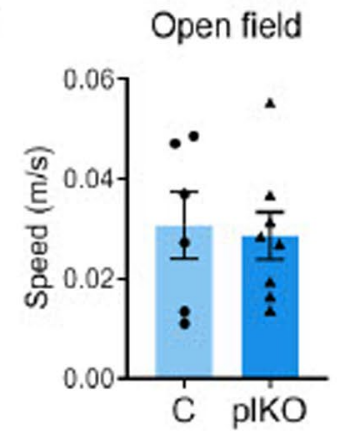

h

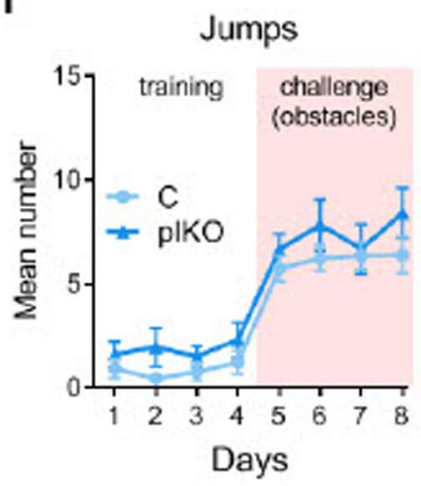

I

Post-perturbation time

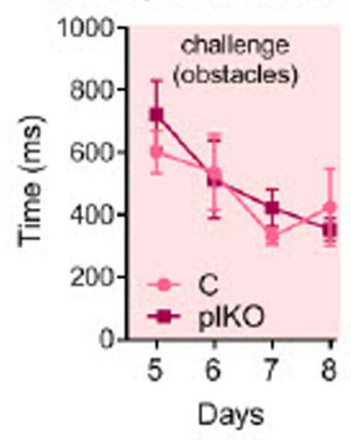

m

Missteps

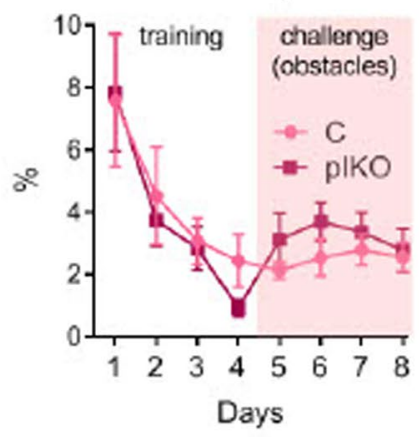

n

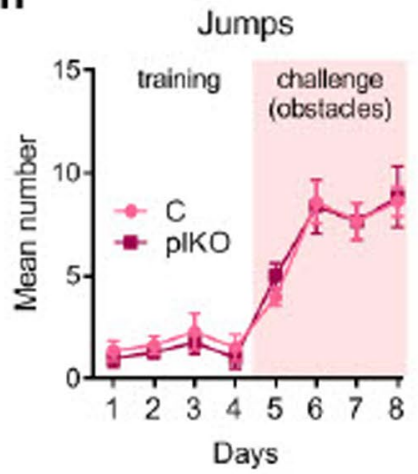

Extended Data Fig. 8 | See next page for caption. 
Extended Data Fig. 8 | Locomotor assessment in the Erasmus ladder at P30. a-h, Performance on the Erasmus ladder. a-b, The analysis of baseline gait and coordination on days 1-4 showed significantly longer steps in adult male plKO mice than in their control littermates. Data is presented as means \pm SEM. Two-way repeated-measures ANOVA with Sidak's multiple comparisons test $\left({ }^{*} p<0.05\right) . n=8 \mathrm{C}$ and 6 plKO. Long steps $=$ Day 1: $p=0.64 ;$ Day 2: $p=0.031$; Day 3: $p=0.039 ;$ Day 4: $p=0.011$. Short steps=Day 1: $p=0.61$; Day 2: $p=0.029 ;$ Day 3: $p=0.039 ;$ Day 4: $p=0.015$. $\mathbf{c}$, Speed on the ladder was unchanged between $C$ and pIKO mice. Data is presented as means \pm SEM. Two-way repeated-measures ANOVA with Sidak's multiple comparisons test $(n=8 \mathrm{C}$ and $6 \mathrm{pIKO})$. d, Locomotor speed was unchanged in the open-field. Data is presented as means \pm SEM. Two-tailed unpaired Student's t test with Welch's correction ( $n=6 \mathrm{C}$ and 8 plKO). e-h, The associative cerebellar learning was tested with the introduction of a conditioned tone and obstacle on days 5-8. The pIKO mice exhibited no motor learning deficit. Data is presented as means \pm SEM. Two-way repeated-measures ANOVA with Sidak's multiple comparisons test $(n=8 \mathrm{C}$ and $6 \mathrm{pIKO})$. i-j, The analysis of baseline gait and coordination on days 1-4 showed no difference in female plKO mice vs their control littermates. Data is presented as means \pm SEM. Two-way repeated-measures ANOVA with Sidak's multiple comparisons test $(n=7 \mathrm{C}$ and 8 PIKO). $\mathbf{k}-\mathbf{n}$, The associative cerebellar learning was tested with the introduction of a conditioned tone and obstacle on days 5-8. The pIKO mice exhibited no motor learning deficit. Data is presented as means \pm SEM. Two-way repeated-measures ANOVA with Sidak's multiple comparisons test $(n=7 \mathrm{C}$ and 8 pIKO). 


\section{ARTICLES}

a

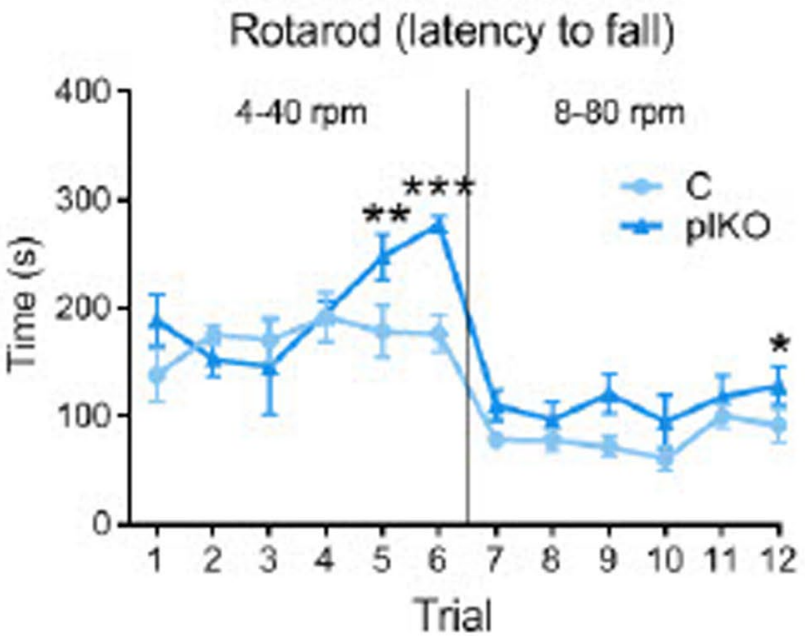

C

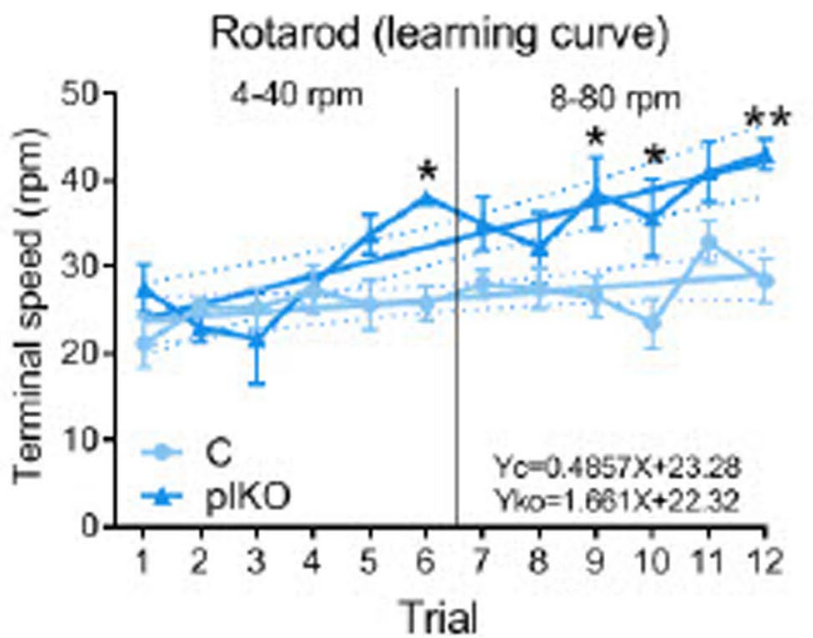

b

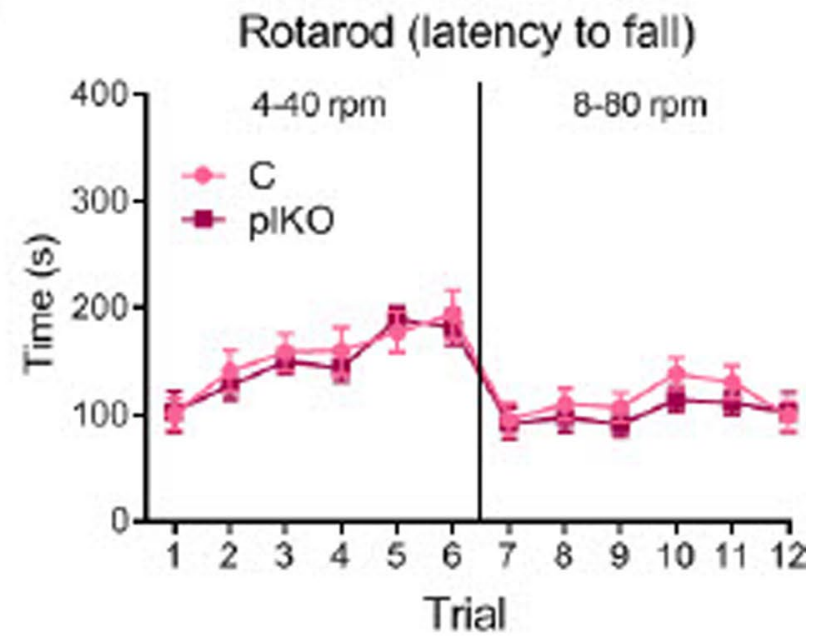

d

Rotarod (learning curve)

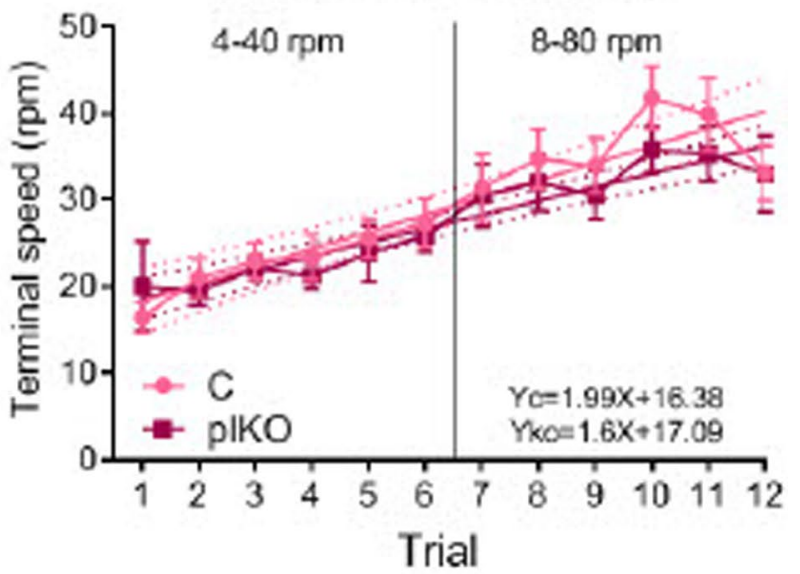

e

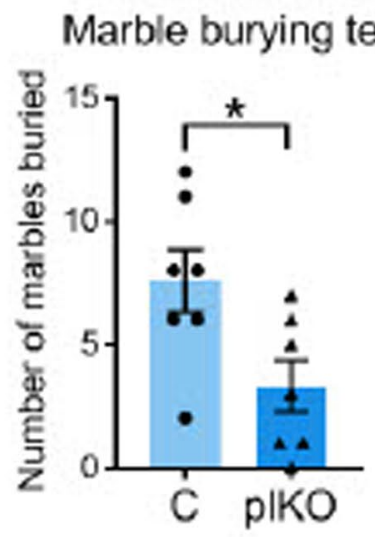

Extended Data Fig. 9 | See next page for caption. 
Extended Data Fig. 9 | Locomotor and balance behavioral testing at P30. a-d, Accelerating rotarod performance at 4 to 40 rpm (trials 1-6) and 8 to $80 \mathrm{rpm}$ (trials 7-12). a-b, Time to fall off is presented at 4 to $40 \mathrm{rpm}$ and 8 to $80 \mathrm{rpm}$ in males (a) and females (b). Data is presented as means \pm SEM. Two-way repeated-measures ANOVA with Sidak's multiple comparisons test ( $\left.{ }^{\star} p<0.05,{ }^{\star \star} p<0.01,{ }^{\star \star \star} p<0.005\right)$. Males: $n=9 \mathrm{C}$ and 7plKO (Trial1: $p=0.4346 ;$ Trial2: $p=0.9961 ;$ Trial3: $p=0.9918 ;$ Trial4: $p>0.9999 ;$ Trial5: $p=0.0386 ;$ Trial6: $p=0.0016 ;$ Trial7: $p=0.9420 ;$ Trial8: $p=0.9995 ;$ Trial9: $p=0.4957$; Trial10: $p=0.9118$; Trial11: $p=0.9377$; Trial12: $p=0.0495$ ); Females: $n=6 C$ and 4 plKO (Any trial: $p>0.98$ ). $c-d$, Terminal speed of rotation on the accelerating rotarod, presented at 4 to $40 \mathrm{rpm}$ (trials 1-6) and 8 to $80 \mathrm{rpm}$ (trials 7-12), was used to calculate learning rate (linear regression). The slopes from $C$ and pIKO are significantly different in males $(c)(F=11.32, D F d=20, p=0.0031)$ but not in females $(d)$. Data is presented as means \pm SEM. Two-way repeated-measures ANOVA with Sidak's multiple comparisons test $\left({ }^{\star} p<0.05,{ }^{\star \star} p<0.01,{ }^{\star \star \star} p<0.005\right)$. Males: $n=9 \mathrm{C}$ and $7 \mathrm{plKO}$ (Trial1: $p>0.99 ;$ Trial2: $p=0.4176 ;$ Trial3: $p=0.0328 ;$ Trial4: $p=0.6343 ;$ Trial5: $p=0.9589 ;$ Trial6: $p=0.0328 ;$ Trial7: $p=0.6343 ;$ Trial8: $p=0.9589 ;$ Trial9: $p=0.0372$; Trial10: $p=0.0328$; Trial11: $p=0.4022$; Trial12: $p=0.0045$ ); Females: $n=6 \mathrm{C}$ and 4 pIKO (Any trial: $p>0.9$ ). e, Marble burying test. Graph represents the number of marbles at least $2 / 3^{\text {rd }}$ buried over $30-\mathrm{min}$. Data is presented as means $\pm \mathrm{SEM}$. Two-tailed unpaired Student's $t$ test with Welch's correction ( $\left.{ }^{\star} p<0.05\right) \cdot n=7$ mice/group $(p=0.023)$. 
a

\section{OL lineage cell density}

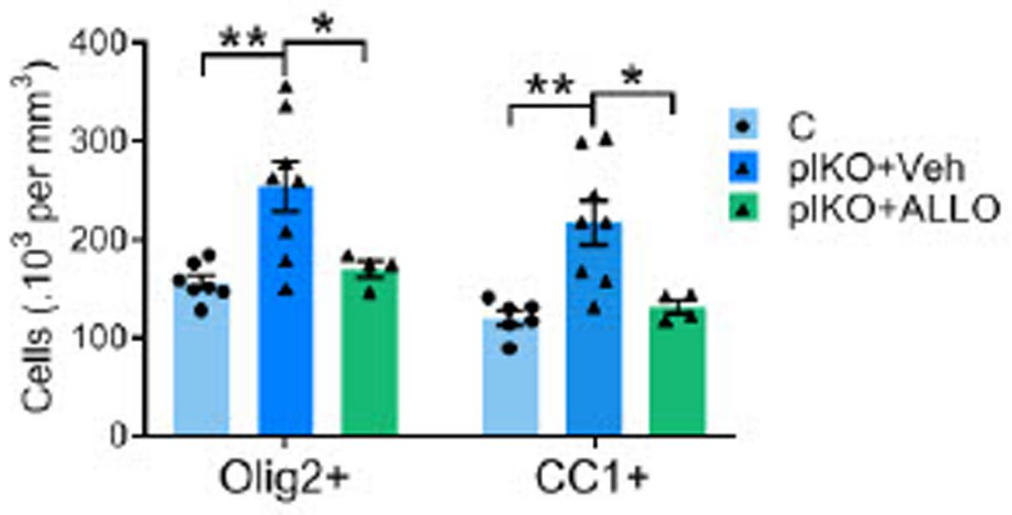

b

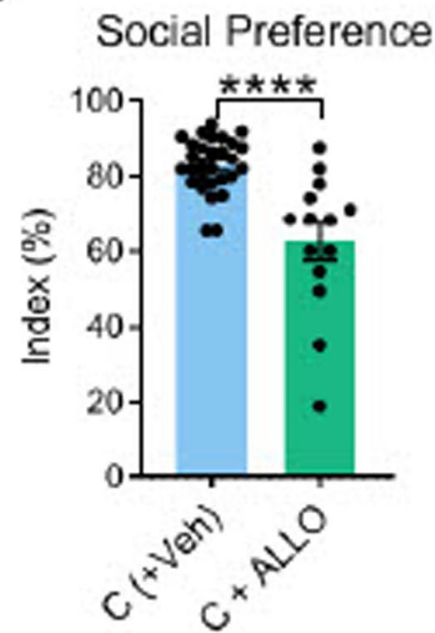

C

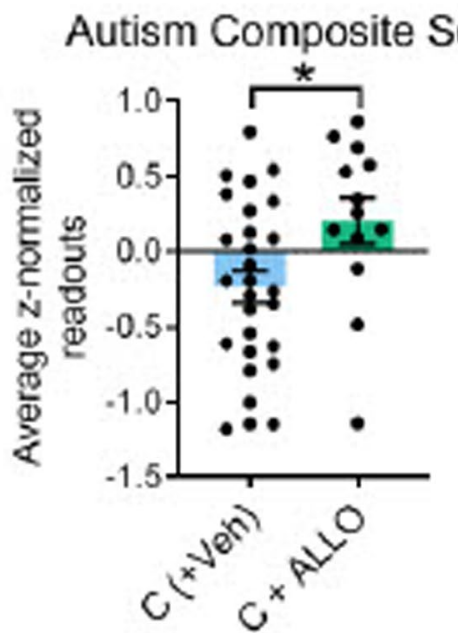

Extended Data Fig. 10 | Cerebellar WM and behavioral impairments in male plKO mice can be prevented by pharmacological treatments at E15.5. a, Quantification of Olig2+ and CC1 + cell densities in the male cerebellar WM $(n=7 C, 8$ pIKO+Veh and 4 pIKO+ALLO). Data presented as means \pm SEM. Two-way ANOVA with Sidak's multiple comparisons test $\left({ }^{\star} p<0.05 ;{ }^{*} p<0.01\right)$. Olig2 $+=C$ vs pIKO+Veh: $p=0.0024 ; C$ vs pIKO+ALLO: $p=0.9982 ; p I K O+V e h$ vs pIKO+ALLO: $p=0.041$. CC1+: $C$ vs pIKO+Veh: $p=0.0014 ; C$ vs pIKO+ALLO: $p=0.9995 ;$ pIKO+Veh vs pIKO+ALLO: $p=0.0354$. b, 3-chamber sociability test. Data is presented as means \pm SEM. Two-tailed unpaired Student's $t$ test with Welch's correction ( $\left.{ }^{\star \star \star *} p<0.001\right)$. $\mathrm{n}=29 \mathrm{C}(+\mathrm{Veh})$ and $14 \mathrm{C}+\mathrm{ALLO}(\mathrm{p}<0.0001) \cdot \mathbf{c}$, ASD composite severity score. Data presented as means \pm SEM. Two-tailed unpaired Student's t test with Welch's correction $\left({ }^{*} p<0.05\right) \cdot n=28 C(+$ Veh $)$ and $13 C+A L L O(p=0.0261)$. 


\section{Reporting Summary}

Nature Research wishes to improve the reproducibility of the work that we publish. This form provides structure for consistency and transparency in reporting. For further information on Nature Research policies, see our Editorial Policies and the Editorial Policy Checklist.

\section{Statistics}

For all statistical analyses, confirm that the following items are present in the figure legend, table legend, main text, or Methods section.

$\mathrm{n} / \mathrm{a}$ Confirmed

$\bigotimes$ The exact sample size $(n)$ for each experimental group/condition, given as a discrete number and unit of measurement

$\square$ A statement on whether measurements were taken from distinct samples or whether the same sample was measured repeatedly

The statistical test(s) used AND whether they are one- or two-sided

Only common tests should be described solely by name; describe more complex techniques in the Methods section.

$\square$ A description of all covariates tested

$\square$ \ A description of any assumptions or corrections, such as tests of normality and adjustment for multiple comparisons

$\square$ A full description of the statistical parameters including central tendency (e.g. means) or other basic estimates (e.g. regression coefficient)

AND variation (e.g. standard deviation) or associated estimates of uncertainty (e.g. confidence intervals)

For null hypothesis testing, the test statistic (e.g. $F, t, r$ ) with confidence intervals, effect sizes, degrees of freedom and $P$ value noted

Give P values as exact values whenever suitable.

Х $\square$ For Bayesian analysis, information on the choice of priors and Markov chain Monte Carlo settings

Х $\square$ For hierarchical and complex designs, identification of the appropriate level for tests and full reporting of outcomes

$\square$ Xstimates of effect sizes (e.g. Cohen's d, Pearson's $r$ ), indicating how they were calculated

our web collection on statistics for biologists contains articles on many of the points above.

\section{Software and code}

Policy information about availability of computer code

Data collection Image acquisition on the scanning electron microscope was done using MAPS Software version 3.7 (ThermoFisher). Image acquisition on the confocal microscope was done with LAS X Life Science Microscope software version 2.7 (Leica Microsystems). Image acquisition on the scanning fluorescent microscope was done using cellSens version 2.3 (Olympus).

FSL software package 5.0.11 (FMRIB, Oxford, UK) was used to generate fractional anisotropy (FA) and mean diffusivity (MD) maps.

Data analysis Data was analyzed using ImageJ 1.53c (NIH), Microsoft Excel 2016 and Graphpad Prism 7. Sequencing data was analyzed using TCC R package (version 1.12.1), Partek Genomics Suite (version 6.6), Ingenuity Pathway Analysis (IPA, Qiagen) and Venny 2.1.

For manuscripts utilizing custom algorithms or software that are central to the research but not yet described in published literature, software must be made available to editors and

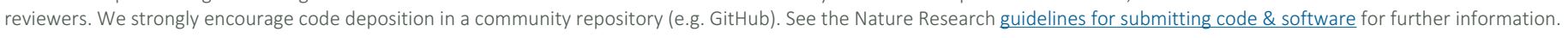

\section{Data}

Policy information about availability of data

All manuscripts must include a data availability statement. This statement should provide the following information, where applicable:

- Accession codes, unique identifiers, or web links for publicly available datasets

- A list of figures that have associated raw data

- A description of any restrictions on data availability

Raw and processed data are available from the corresponding authors upon request. 
Please select the one below that is the best fit for your research. If you are not sure, read the appropriate sections before making your selection.

\ Life sciences

Behavioural \& social sciences Ecological, evolutionary \& environmental sciences

For a reference copy of the document with all sections, see nature.com/documents/nr-reporting-summary-flat.pdf

\section{Life sciences study design}

All studies must disclose on these points even when the disclosure is negative.

Sample size $\quad$ The sample sizes are shown in the legends and chosen to meet or exceed sample sizes typically used in the field (El Khordi et al., 2013; Lerch et al., 2011; Zonouzi et al., 2015). Preliminary experiments were performed when possible to determine requirements for sample size, taking into consideration resources available and ethical, reductionist animal use. Sample size was somewhat limited by the litter sizes and Mendelian ratios of complex genotypes but mice from at least 3 litters were used to overcome this limitation.

Data exclusions - Effect of muscimol treatment on social behavior: one mouse was excluded because it refused to get out of the middle chamber in the 3chamber equipment.

- Erasmus Ladder: 5 mice were removed from the experiment because they refused to perform the 42 runs in a row per day required for the

2-way Repeated Measures ANOVA analysis.

- Western blot on human tissue: One sample was excluded due to poor tissue quality.

- ROUT methodology was utilized to determine outliers.

Replication Every experiment was performed on animals from at least 3 different litters. Two technical replicates were performed for RT-PCR and Western bot analyses on mouse and human samples. All attempts at replication were successful.

Randomization Sample assignment to groups was not randomized, since it relies on their known genotype (C vs plKO mice) or gestational age (T and PT infants). Internal controls (housekeeping proteins and genes) were used in the molecular and biochemical experiments.

\section{Reporting for specific materials, systems and methods}

We require information from authors about some types of materials, experimental systems and methods used in many studies. Here, indicate whether each material, system or method listed is relevant to your study. If you are not sure if a list item applies to your research, read the appropriate section before selecting a response.

\begin{tabular}{l|ll}
\multicolumn{2}{l}{ Materials \& experimental systems } & Methods \\
\hline n/a Involved in the study & $\square$ Antibodies & $\square$ Eukaryotic cell lines \\
$\square$ Palaeontology and archaeology & $\bigotimes$ Animals and other organisms \\
$\square$ & $\bigotimes$ Human research participants \\
$\square$ & $\square$ Clinical data \\
$\square$ & $\square$ Dual use research of concern
\end{tabular}

\section{Antibodies}

Antibodies used

Antibodies were used as follows for immunohistochemistry: : rabbit anti-MBP (Abcam, Cambridge, MA, USA; \#ab40390; 1:500), mouse anti-APC clone CC1 (EMD Millipore, Burlington, NA, USA; \#MABC20; 1:500), rabbit anti-Olig2 (Abcam; \#ab9610, 1:500), mouse anti-neuronal nuclei (NeuN) (Millipore; \#MAB377; 1:500), goat anti-NeuroD1 (RD Systems, Minneapolis, MN, USA; \#AF2746; 1:500), rabbit anti-calbindin (Swant, Marly, Switzerland; \#CB38, 1:1000), rat anti-mouse PDGFRo (CD140a; BD Biosciences, San Jose, CA, USA; \#17-1401-81; 1:500), chicken anti-GFP (Abcam; \#ab13970; 1:500), , donkey anti-mouse Alexa-488 (Invitrogen; \#A-21202), donkey anti-rabbit Alexa-488 (Invitrogen; \#A-21206), donkey anti-mouse Alexa-555 (Invitrogen; \#A-31570), donkey anti-rabbit Alexa-555 (Invitrogen; \#A-31572), donkey anti-mouse Alexa-647 (Invitrogen; \#A-31571), and donkey anti-rabbit Alexa-647 (Invitrogen; \#A-31573).

Antibodies were used as follows for Western blots: rabbit anti-MBP (Abcam; \#ab40390; 1:500), rabbit anti-MAG (Thermo Fisher Scientific; \#PA5-79620; 1:500), rabbit anti-MOG (Abcam; \#ab32760; 1:500), mouse anti-cofilin-1 (Cfl1) (Santa Cruz Biotechnology, Dallas, TX, USA; \#sc-53934; 1:500), mouse anti-prosaposin (PSAP) (Santa Cruz Biotechnology; \#sc-390184; 1:500), rabbit anti-hnRNPk (R332) (Cell Signaling Technology; \#4675; 1:500) and rabbit anti-GAPDH (Cell Signaling Technology, Danvers, MA, USA; \#5174; 1:2000). 


\section{Animals and other organisms}

Policy information about studies involving animals; ARRIVE guidelines recommended for reporting animal research

Laboratory animals The strains used in this study were: Cyp-19a-Cre, Akr1c14-floxed and ROSA26-YFP mice. Males and females were collected at embryonic days: E12.5, E14.5, E15.5, E16.5, E17.5, E19.5 and postnatal days P30.

Mice were housed in a climate controlled facility with a fixed 12/12 hour light cycle with ad libitum access to food and water. All behavioral experiments were conducted between 8 am and $5 \mathrm{pm}$.

Wild animals The study did not involve wild animals.

Field-collected samples The study did not involve samples collected from fields.

Ethics oversight $\quad$ All the procedures on experimental animals were performed in accordance with the protocols approved by the Institutional Animal Care and Use Committee at Children's National Medical Center (protocol \# 30534 (PI: Penn)) and at Columbia University Medical Center (protocols \# AC-AABE 6553 (PI: Penn) and AC-AABF 5550 (PI: Yang)).

Note that full information on the approval of the study protocol must also be provided in the manuscript.

\section{Human research participants}

\section{Policy information about studies involving human research participants}

Population characteristics

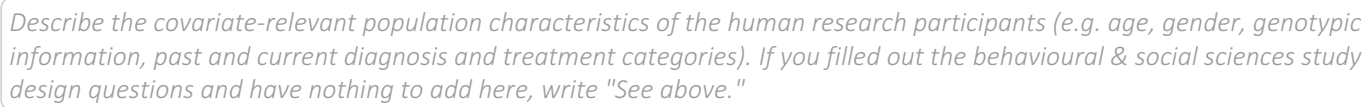
information, past and current diagnosis and treatment categories). If you filled out the behavioural \& social sciences study design questions and have nothing to add here, write "See above."

Recruitment

Describe how participants were recruited. Outline any potential self-selection bias or other biases that may be present and how these are likely to impact results.

Ethics oversight

Identify the organization(s) that approved the study protocol.

Note that full information on the approval of the study protocol must also be provided in the manuscript.

\section{Magnetic resonance imaging}

\section{Experimental design}

Design type

Design specifications

Behavioral performance measures

MRI was used to examine the structural integrity of white matter tracts in the brains of $4 \%$ paraformaldehyde fixed brains.

\section{$4 \%$ paraformaldehyde perfused brains}

no tasks performed during

\section{Acquisition}

Imaging type(s)

Field strength

Sequence \& imaging parameters

Area of acquisition

Diffusion MRI

\used structural/diffusion

$7 T$

Parameters for the DTI sequence were as follows: TR of $270 \mathrm{~ms}$, echo train length of 6, first TE of $32 \mathrm{~ms}$, and a TE of 10 $\mathrm{ms}$ for the remaining 5 echoes, 1 average, FOV of $14 \mathrm{~mm} \times 14 \mathrm{~mm} \times 25 \mathrm{~mm}$ and a matrix size of $180 \times 180 \times 324$ resulting in an image with $78 \mu \mathrm{m}$ isotropic voxels. Five $b=0 \mathrm{~s} / \mathrm{mm} 2$ images and 30 high b value $(b=2147 \mathrm{~s} / \mathrm{mm} 2)$ in 30 different directions were acquired, using the Jones scheme. Total imaging time was approximately 12 hours.

\section{whole brain}

Not used

Parameters Parameters for the DTI sequence were as follows: TR of $270 \mathrm{~ms}$, echo train length of 6, first TE of $32 \mathrm{~ms}$, and a TE of $10 \mathrm{~ms}$ for the remaining 5 echoes, 1 average, FOV of $14 \mathrm{~mm} \times 14 \mathrm{~mm} \times 25 \mathrm{~mm}$ and a matrix size of $180 \times 180 \times 324$ resulting in an image with 78 $\mu \mathrm{m}$ isotropic voxels. Five $b=0 \mathrm{~s} / \mathrm{mm} 2$ images and 30 high $b$ value $(b=2147 \mathrm{~s} / \mathrm{mm} 2)$ in 30 different directions were acquired, using the Jones scheme. Total imaging time was approximately 12 hours.

\section{Preprocessing}


Normalization

Normalization template

Volume censoring
To assess any changes to the mouse brains due to genotype and sex, the $b=0 \mathrm{~s} / \mathrm{mm} 2$ images were registered linearly (6 followed by 12 parameter) and non linearly together. A combination of mni_autoreg tools and ANTS (advanced normalization tools) were used to perform the registrations.

A population atlas, representing the average anatomy of the study samples, was created when all scans were resampled with an appropriate transform. The final registration results are the individual images deformed into alignment with one another in an unbiased manner. Warping an available classified MRI mouse brain atlas onto the population average allows for significant differences in the diffusion measures of segmented structures to be calculated.

N/A

N/A

Statistical modeling \& inference

Model type and settings

This study examined fixed brains, without any behavioral tasks, no statistical modeling was involved.

Effect(s) tested

The analysis of the individual DTI parameters compare intensity differences of FA between genotype and sex, in different ROIs, using 3-way ANOVA.

Specify type of analysis:

Whole brain $\bigotimes$ ROI-based $\square$ Both

- Cerebellum-related tracts

Anatomical location(s) - Other major cerebral tracts: anterior commissure, posterior commissure, corpus callosum, internal capsule, fornix, mammillothalamic tract, corticospinal tract, cerebral peduncle.

Statistic type for inference (See Eklund et al. 2016)

Correction

Models \& analysis

\begin{tabular}{l|l} 
In & Involved in the study \\
$\square$ & Functional and/or effective connectivity
\end{tabular}

